University of Tennessee Health Science Center UTHSC Digital Commons

\title{
$5-2015$
}

\section{Structural-based Investigation of FAK and P130Cas in Focal Adhesion}

Chi Zhang

University of Tennessee Health Science Center

Follow this and additional works at: https://dc.uthsc.edu/dissertations

Part of the Medical Cell Biology Commons

\section{Recommended Citation}

Zhang, Chi , "Structural-based Investigation of FAK and P130Cas in Focal Adhesion" (2015). Theses and Dissertations (ETD). Paper 313. http://dx.doi.org/10.21007/etd.cghs.2015.0373.

This Dissertation is brought to you for free and open access by the College of Graduate Health Sciences at UTHSC Digital Commons. It has been accepted for inclusion in Theses and Dissertations (ETD) by an authorized administrator of UTHSC Digital Commons. For more information, please contact jwelch30@uthsc.edu. 


\title{
Structural-based Investigation of FAK and P130Cas in Focal Adhesion
}

\begin{abstract}
Cell migration is an integrated multistep process accompanying us throughout life, from birth to death. It contributes to a variety of biological activities and has been implicated in many pathological conditions, including cancer, primary immunodeficiency diseases, vascular diseases, and mental retardation. Cell migration has been considered to be a three-step cyclic process including polarization, protrusion and retraction. The initiation and extension of the protrusion is driven by actin polymerization, and is stabilized by attaching to the extracellular matrix (ECM) or adjacent cells. The cell-ECM attachment sites are named focal adhesions (FAs), which are dynamic, large protein complexes containing more than 100 proteins. p130cas is a Src substrate localized in FAs that functions in integrin signaling to promote cell motility, invasion, proliferation and survival. The Src homology $3(\mathrm{SH} 3)$ domain of p130Cas can directly interact with Focal Adhesion Kinase (FAK), which is a non-receptor tyrosine kinase that prominently locates in FAs. FAK serves the dual functions of recruiting p130Cas to FAs and recruiting Src kinase to phosphorylate the p130Cas substrate domain (SD) to promote downstream signaling. p130Cas targeting to focal adhesions and undergoing tyrosine phosphorylation in responding to adhesion signal plays an important role in cell motility, invasion and survival. Mice lacking p130Cas revealed embryonic lethality due to defects associated with the disorganized actin cytoskeleton. Although the importance of p130Cas localization and phosphorylation is well established, how it targets FAs has not been elucidated. Previous study demonstrated that, the N-terminal SH3 domain is critical for p130Cas localization through interacting with FAK. Deletion of SH3 domain and Cas-family C-terminal homology domain (CCHD) impairs p130Cas FA localization ability. The $\mathrm{C}$-terminal region of $\mathrm{p} 130 \mathrm{Cas}$ has been proposed as a Cas family homology domain, which may adopt a similar folding formation to the FAK C-terminal Focal Adhesion Targeting (FAT) domain. The nature of the target protein for the p130Cas CCHD in promoting FAs localization is unclear. In this dissertation we study the FAs localization of both FAK and p130Cas for understanding the detailed mechanisms. I proposed to develop the helical constrained peptidomimetics for targeting the FAT domain of FAK as inhibitor in order to address how FAK interacts with paxillin through its FAT domain associating with LD motifs of paxillin. The peptidomimetic inhibitors provide opportunity as potential therapeutic targets to regulate FAK activity in FAs. In the second part I identified the binding partner of p130Cas CCHD in FAs and solved the structure of p130Cas CCHD complex with paxillin LD1 that first demonstrated the detailed mechanism of CCHD playing an important role in p130Cas FAs targeting.
\end{abstract}

\section{Document Type}

Dissertation

Degree Name

Doctor of Philosophy (PhD)

Program

Biomedical Sciences

Research Advisor

Jie J. Zheng, Ph.D.

\section{Keywords}

FAK, Focal adhesion, Helical constrained peptide, NMR, P130Cas, Paxillin

\section{Subject Categories}

Medical Cell Biology | Medical Sciences | Medicine and Health Sciences 
Comments

One year embargo expired May 2016

This dissertation is available at UTHSC Digital Commons: https://dc.uthsc.edu/dissertations/313 


\title{
Structural-based Investigation of FAK and P130Cas in Focal Adhesion
}

\author{
A Dissertation \\ Presented for \\ The Graduate Studies Council \\ The University of Tennessee \\ Health Science Center
}

In Partial Fulfillment

Of the Requirements for the Degree

Doctor of Philosophy

From The University of Tennessee

By

Chi Zhang

May 2015 
Copyright (C) 2015 by Chi Zhang. All rights reserved. 


\section{DEDICATION}

This dissertation is dedicated to my parents, Mrs. Jianfei Wang and Mr. Chao Zhang, my wife, Dr. Xi Wang, and my daughter, Aimee Zhang for their love and support 


\section{ACKNOWLEDGEMENTS}

I would like to acknowledge all the people who have helped and supported me throughout my graduate studies. Firstly, I would like to express my deepest gratitude to my advisor Dr. Jie Zheng, for bringing me to the lab, and all the guidance, caring, patience, mentorship, and providing me with an excellent atmosphere for doing research throughout my graduate studies. I would also thank everyone in the Zheng laboratory, especially the lab manger Cristina Guibao, whose hard working provides a solid theoretical foundation for my project, Dr. Ezelle McDonald, Dr. Hojin Lee Dr. Ju Bao, Dr. Murugendra Vanarotti, Dr. Rana Rajashree and Dr. Xinxin Zhang for technical assistance and helpful suggestions.

I would like to thank my committee members, Dr. Eric Enemark, Dr. David Nelson, Dr. Susan Senogles, and Dr. Taosheng Chen for their direction, dedication, and invaluable advice along this project for the past several years. Thank them for encouraging and helping me to develop my knowledge and background in biology and biochemistry.

I would like to thank Dr. Darcie miller, from the Crystallography facility, Dr. Grace Royappa and Dr. Weixin Zhang from the NMR facility for all the technical supports along the way.

Finally, I would like to thank my parents for bringing me to this world, raising me up, supporting my education, and most importantly giving me love all the time. I also want to thank my loving wife, Dr. Xi Wang, for his encouragement and support during the past five years, making my life in a foreign country more meaningful and happy. 


\begin{abstract}
Cell migration is an integrated multistep process accompanying us throughout life, from birth to death. It contributes to a variety of biological activities and has been implicated in many pathological conditions, including cancer, primary immunodeficiency diseases, vascular diseases, and mental retardation. Cell migration has been considered to be a three-step cyclic process including polarization, protrusion and retraction. The initiation and extension of the protrusion is driven by actin polymerization, and is stabilized by attaching to the extracellular matrix (ECM) or adjacent cells. The cell-ECM attachment sites are named focal adhesions (FAs), which are dynamic, large protein complexes containing more than 100 proteins. p130cas is a Src substrate localized in FAs that functions in integrin signaling to promote cell motility, invasion, proliferation and survival. The Src homology 3 (SH3) domain of p130Cas can directly interact with Focal Adhesion Kinase (FAK), which is a non-receptor tyrosine kinase that prominently locates in FAs. FAK serves the dual functions of recruiting p130Cas to FAs and recruiting Src kinase to phosphorylate the p130Cas substrate domain (SD) to promote downstream signaling. p130Cas targeting to focal adhesions and undergoing tyrosine phosphorylation in responding to adhesion signal plays an important role in cell motility, invasion and survival. Mice lacking p130Cas revealed embryonic lethality due to defects associated with the disorganized actin cytoskeleton. Although the importance of p130Cas localization and phosphorylation is well established, how it targets FAs has not been elucidated. Previous study demonstrated that, the N-terminal SH3 domain is critical for p130Cas localization through interacting with FAK. Deletion of SH3 domain and Casfamily C-terminal homology domain (CCHD) impairs p130Cas FA localization ability. The C-terminal region of $\mathrm{p} 130 \mathrm{Cas}$ has been proposed as a Cas family homology domain, which may adopt a similar folding formation to the FAK C-terminal Focal Adhesion Targeting (FAT) domain. The nature of the target protein for the p130Cas CCHD in promoting FAs localization is unclear. In this dissertation we study the FAs localization of both FAK and p130Cas for understanding the detailed mechanisms. I proposed to develop the helical constrained peptidomimetics for targeting the FAT domain of FAK as inhibitor in order to address how FAK interacts with paxillin through its FAT domain associating with LD motifs of paxillin. The peptidomimetic inhibitors provide opportunity as potential therapeutic targets to regulate FAK activity in FAs. In the second part I identified the binding partner of p130Cas CCHD in FAs and solved the structure of p130Cas CCHD complex with paxillin LD1 that first demonstrated the detailed mechanism of CCHD playing an important role in p130Cas FAs targeting.
\end{abstract}




\section{TABLE OF CONTENTS}

CHAPTER 1. INTRODUCTION ..................................................................................

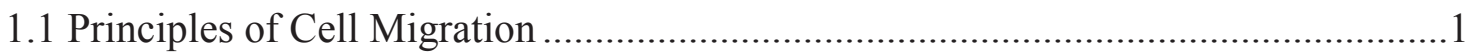

1.1.1 Establishment of Cell Polarization .................................................................

1.1.2 Extension of the Protrusion to Adhere ................................................................

1.1.3 Adhesion Disassembly and Retraction of the Cell Rear .................................2

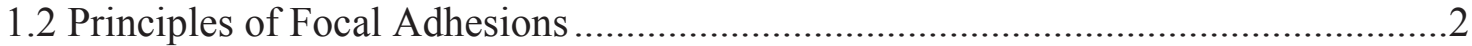

1.2.1 Cell Signaling Through FAs..........................................................................

1.2.2 Key Components of FAs .........................................................................

1.2.2.1 Focal adhesion kinase ..................................................................... 5

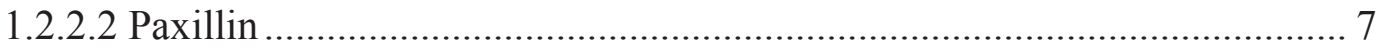

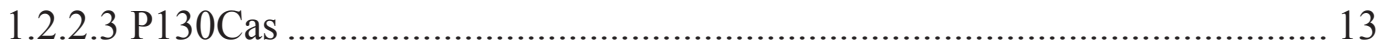

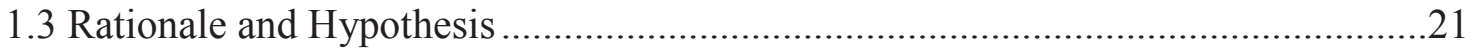

CHAPTER 2. METHODS........................................................................................23

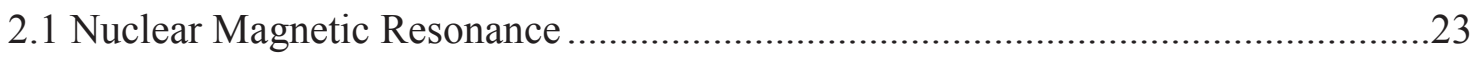

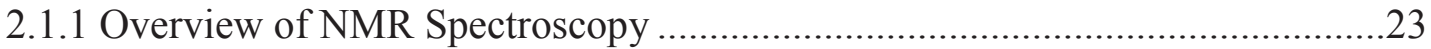

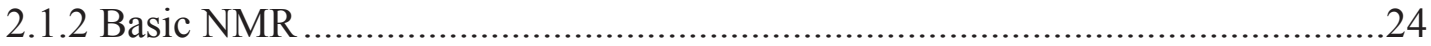

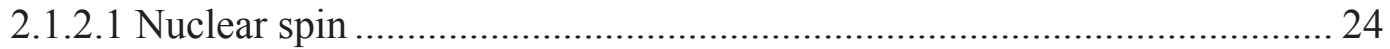

2.1.2.2 Transition energy and absorption of radiation ...................................... 26

2.1.2.3 Pulsed Fourier transform NMR spectroscopy....................................... 26

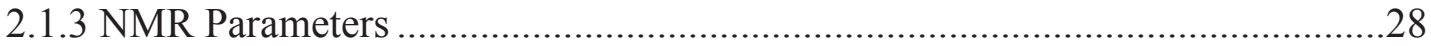

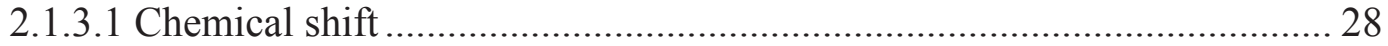

2.1.3.2 Spin-spin coupling ......................................................................... 28

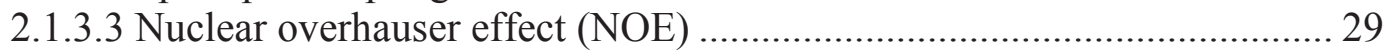

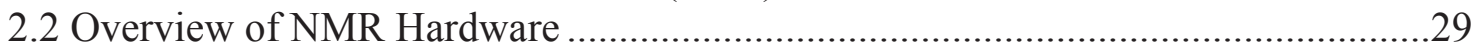

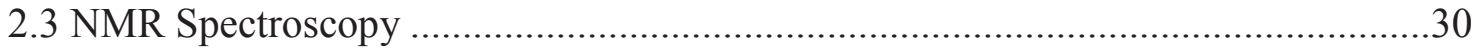

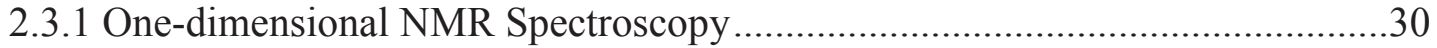

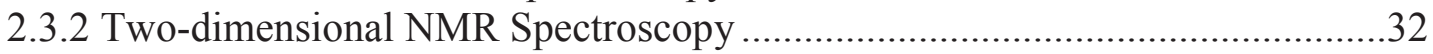

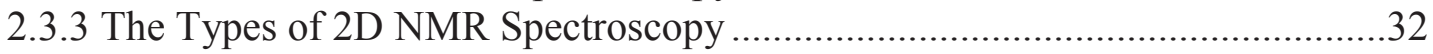

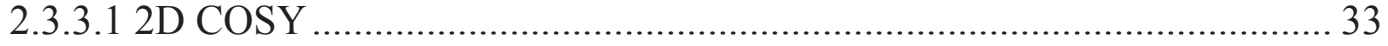

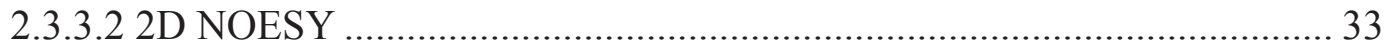

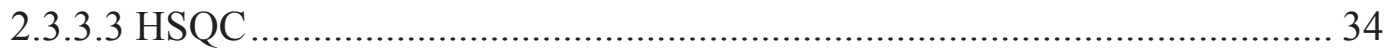

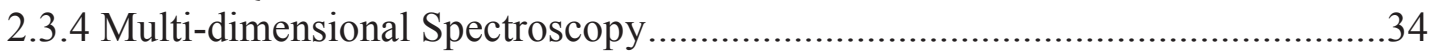

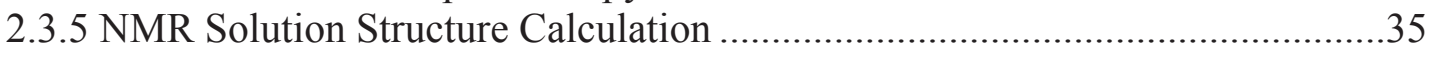

CHAPTER 3. STUDY OF HELICAL CONSTRAINED PEPTIDE ..........................37

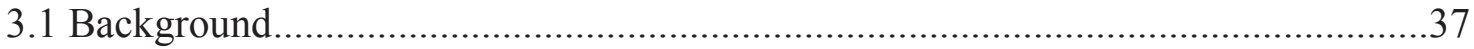

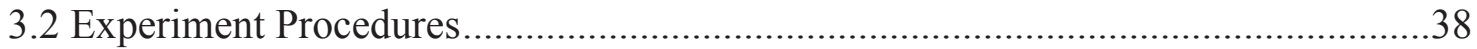

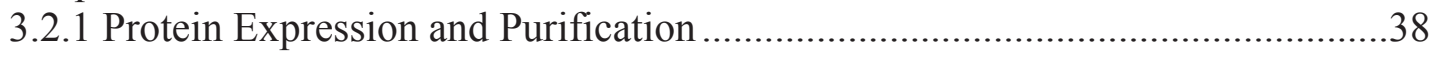

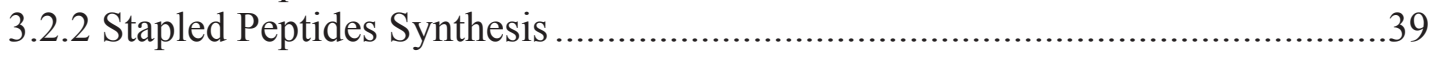

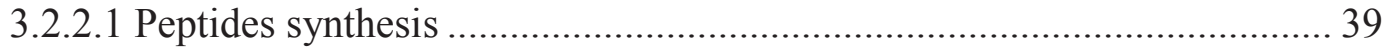

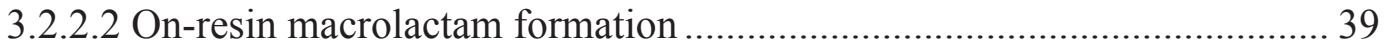

3.2.2.3 Fluorescent labeling ................................................................. 40 
3.2.2.4 Peptide cleavage and purification ........................................................ 40

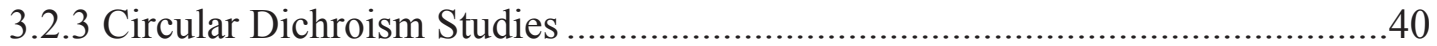

3.2.4 Isothermal Titration Calorimetry Studies .......................................................40

3.2.5 Fluorescent Polarization...........................................................................4

3.2.6 NMR Spectroscopy and Chemical Shift Perturbation......................................41

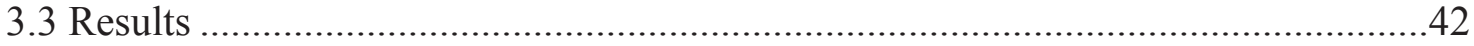

3.3.1 Single Binding Site FAT for Studying Helical Constrained LD4 Peptides ......42

3.3.2 Comparison of Structure of FAT-LD2 and FAT/LD2 Complex .......................44

3.3.3 Design of Helical Constrained LD4 Peptide .......................................................44

3.3.4 Constrained LD4 Peptides Have an Enhanced Helix Propensity........................47

3.3.5 Helical Constrained LD4 Peptides Show Enhanced Binding Affinity to

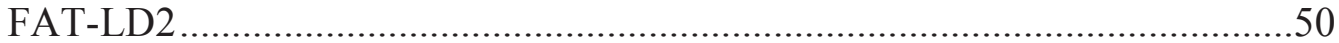

3.3.6 Enhanced Association of Cross-linked LD4 Peptides with FAT-LD2 Validated by Chemical Shift Perturbation..........................................................53

3.3.7 Helical Constrained LD4 Peptides Can Compete with a WT-LD4 Peptide

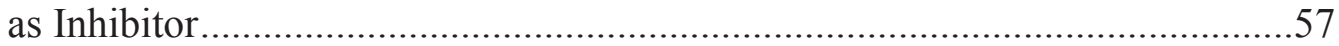

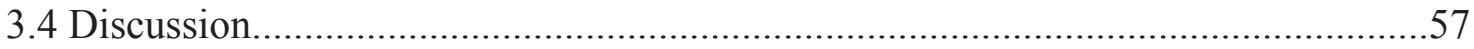

\section{CHAPTER 4. STRUCTURE DETERMINATION OF P130CAS CAS FAMILY C-TERMINAL HOMOLOGY DOMAIN ............................................................62}

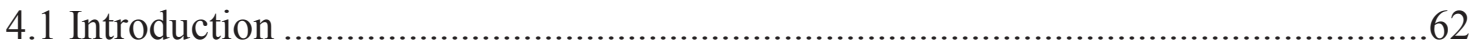

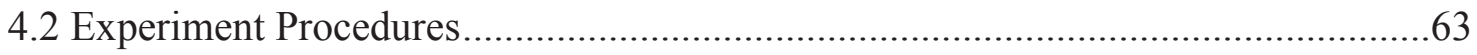

4.2.1 Protein Expression and Purification .........................................................63

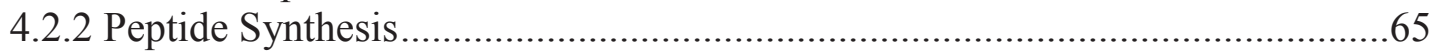

4.2.3 Isothermal Titration Calorimetry Studies ........................................................65

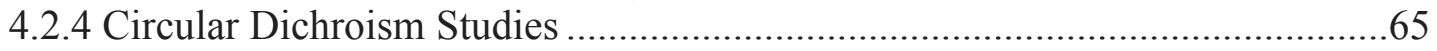

4.2.5 NMR Spectroscopy and Backbone Assignment …………………………......65

4.2.6 Crystallization, Structure Determination and Model Quality ………….............66

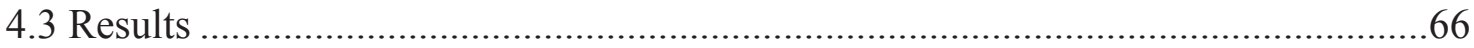

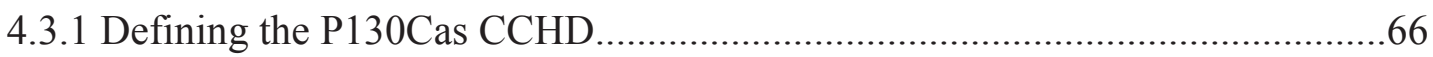

4.3.2 ITC Studies of the Paxillin LD Motifs Binding to P130Cas CCHD ..................67

4.3.3 P130Cas CCHD Stabilized in Complex with Paxillin LD1 ...............................71

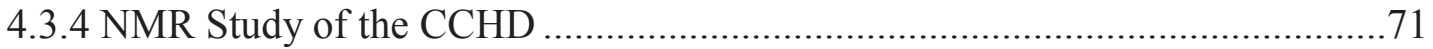

4.3.5 Crystal Structure of the CCHD Complex with LD1 .........................................74

4.3.6 LD1 Motif of Paxillin Binds to CCHD at the H2/H3 Surface ..........................78

4.3.7 Comparison of P130Cas FAK with the FAK FAT Domain ...............................78

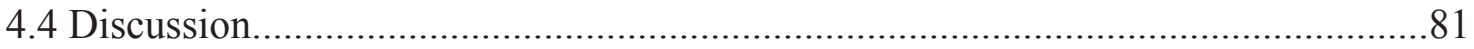

CHAPTER 5. IMPLICATIONS AND FUTRE DIRECTIONS ....................................83

5.1 Helical Constrained Peptide Targets FAK FAs Localization as an Inhibitor ...........83

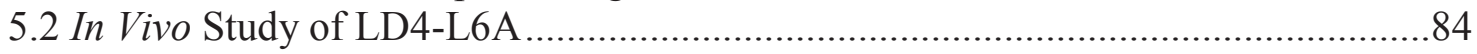

5.3 The Complex Structure of P130Cas CCHD with Paxillin LD1 Motif ......................84

5.4 Unanswered Question of Regulating P130Cas Activity Through CCHD.................86

LIST OF REFERENCES .......................................................................................................89 
VITA 


\section{LIST OF TABLES}

Table 2-1. Properties of nuclei important in protein NMR ...........................................27

Table 3-1. Binding affinity of LD4 peptides to FAT-LD2 ...........................................52

Table 4-1. Data collection and refinement statistics. ..................................................... 75 


\section{LIST OF FIGURES}

Figure 1-1. Molecular architecture of focal contacts.......................................................

Figure 1-2. Focal adhesion kinase domain structure and phosphorylation sites................6

Figure 1-3. The paxillin family and domain structure. ................................................8

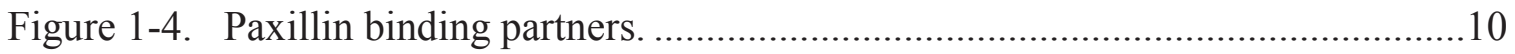

Figure 1-5. Paxillin phosphorylation and function. ………………...........................12

Figure 1-6. The structural characteristics and interacting proteins of $\mathrm{p} 130 \mathrm{Cas}$..............15

Figure 2-1. Nuclear spin precession and energy splitting in presenting external

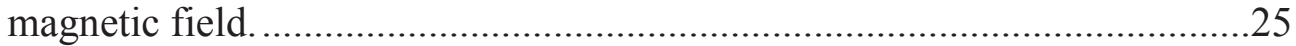

Figure 2-2. Overview of NMR spectroscopy hardware...................................................31

Figure 3-1. Single binding site construct FAT-LD2 ..................................................43

Figure 3-2. Hydrocabon stapling for constraining the helical structure of LD4

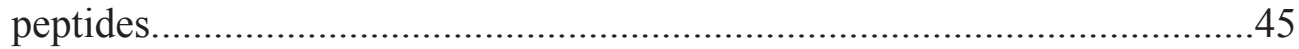

Figure 3-3. LD4 motif of paxillin binds to FAT of FAK driven by hydrophobic interaction.

Figure 3-4. Group one helical constrained LD4 peptides with no or minor contact

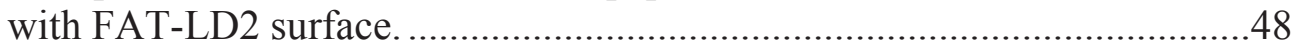

Figure 3-5. Group two helical constrained LD4 with predicted interference of FATLD2 surface.

Figure 3-6. Helical constrained LD4 peptide shows enhanced helix propensity in solution.

Figure 3-7. LD4-L6A significantly enhanced binding to FAT-LD2 based on chemical shift perturbation.

Figure 3-8. LD4-L6A has higher binding affinity to FAT-LD2 comparing with WTLD4.

Figure 3-9. LD4-L6A binds to FAT-LD2 competitively with WT-LD4. .58

Figure 3-10. Helical constrained LD4 peptides can disrupt the FAT-LD2/LD4 complex.

Figure 4-1. Sequence analysis and secondary structure prediction of CCHD. 


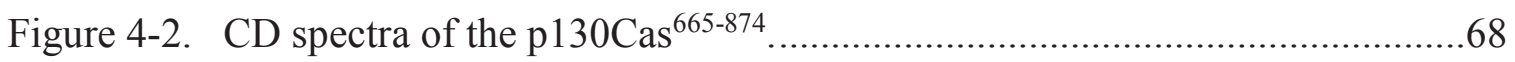

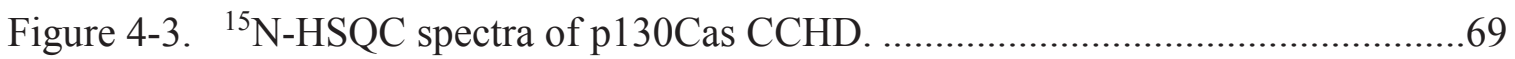

Figure 4-4. ITC titrations show LD1 of paxillin can bind to CCHD of p130Cas............70

Figure 4-5. CD spectra of $\mathrm{p} 130 \mathrm{Cas}{ }^{665-874}$ with or without LD1 peptide. .......................72

Figure 4-6. ${ }^{15} \mathrm{~N}-\mathrm{HSQC}$ spectra of $\mathrm{p} 130 \mathrm{Cas}$ CCHD complex with paxillin LD1............73

Figure 4-7. Crystal structure of p130Cas CCHD complex with paxillin LD1. ..............76

Figure 4-8. 3D structure of the CCHD hydrophobic core. ........................................77

Figure 4-9. Representative electron density for $130 \mathrm{Cas}^{738-874} / \mathrm{LD} 1$.............................79

Figure 4-10. Elucidation of differences between the H1/H4 site on FAT and CCHD.....80

Figure 5-1. Confocal microscopy of helical constrained LD4-S12K addition to NIH-

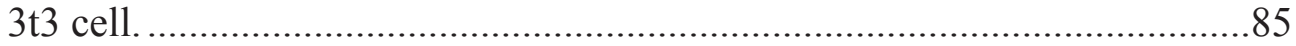

Figure 5-2. Comparing the CCHD/LD1 structure with CCHD/NSP3 _.........................87 


\section{LIST OF ABBREVIATIONS}

ALL

aPKC

BCAR

Cas

CASS4

CCHD

CDK5

CML

DOCK

ECM

EFS

EGF

EGFR

ERBB2

ERK

FAK

FAs

FAT

FERM

FRNK

GAPs

GEFs

GIT

IGF1

ILK

JNK

LIBS

LIM

MAPK

MDCK

MMP

MTOC

NEDD9

PAK

PDGF

PDGFR

PIP3

PIX

PKC

PPR

RACK1

SBD

SD acute lymphoblastic leukemia

atypical protein kinase $\mathrm{C}$

breast cancer anti-estrogen resistance gene

CAS-associated substrate

Cas scaffolding protein family member 4

C-terminal Homology domain

cyclin-dependent kinase 5

chronic myelogenous leukemia

dedicator of cytokinesis

extracellular matrix

embryonal Fyn-associated substrate

epidermal growth factor

epidermal growth factor receptor

receptor tyrosine-protein kinase erbB2

extracellular signal regulated kinase

focal adhesion kinase

Focal adhesions

focal adhesion-targeting

protein 4.1, ezrin, radixin and moesin homology

focal adhesion kinase-related nonkinse

GTPase-activating proteins

guanine-nucleotide-exchange factors

G protein-coupled receptor (GPCR) kinase-interacting protein

insulin-like growth factor I

integrin-linked kinase

Jun N-terminal kinase

ligand induced binding site

Lin11, Isl-1, Mec-3

mitogen activated protein kinase

Madin-darby canine kidney

matrix metalloproteinase

microtubule organizing center

neural precursor cell expressed, developmentally down-regulated 9

p21-activated kinase

platelet-derived growth factor

platelet-derived growth factor receptor

phosphoinositides PtdIns(3,4,5)P3

PAK-ineracting exchanger factor

protein kinase $\mathrm{C}$

proline-rich region

receptor for activated $\mathrm{C}$ kinase 1

Src-binding domain

substrate domain 
SFK

$\mathrm{SH}$

VEGFR

VEGF
Src family kinase

Src homology

vascular endothelial growth factor receptor

vascular endothelial growth factor 


\section{CHAPTER 1. INTRODUCTION}

\subsection{Principles of Cell Migration}

Cell migration is an integrated multistep process accompanying us throughout life, from birth to death. It contributes to a variety of biological activities, such as leukocyte trafficking in immune surveillance, migration during embryonic morphogenesis, and movements involved in tissue regeneration and repair [1-3]. Aberrant cell migration has been implicated in many pathological conditions, including cancer, primary immunodeficiency diseases, vascular diseases, and mental retardation [2]. Therefore, there has been great interest in understanding and targeting cell migration to treat human diseases.

Cell migration has been considered to be a multistep cyclic process: 1) establishment of cell polarization, 2) extension of the protrusion to adhere, 3 ) retraction at the rear of the cell [2].

\subsubsection{Establishment of Cell Polarization}

Cell polarization is a keystone of migration, as it differentiates the front and the back of a moving cell and tells it where to go. Various cellular signaling events contribute to the initiation and maintenance of cell polarity. For example, during chemotaxis, localized activation of phosphoinositide3-kinases (PI3Ks) leads to accumulation of phosphoinositides PtdIns(3,4,5)P3 (PIP3) and PtdIns(3,4)P2 [PI(3,4)P2] at the leading edge of the cell, resulting in localization of small Rho GTPase Rac activation at the edge [4]. Once Rac is activated, several feedback loops are established to maintain polarity [57] During wounding, the interaction between integrins and extracellular matrix results in the activation and accumulation of $\mathrm{Cdc} 42$ at the leading edge of the cell, which recruits and activates protein complex PAR6/PAR3/aPKC (atypical protein kinase $\mathrm{C}$ ), orienting the microtubule organizing center (MTOC) and Golgi apparatus toward the leading edge to induce polarity [8].

\subsubsection{Extension of the Protrusion to Adhere}

The initiation and extension of the protrusion is driven by actin polymerization. The organization of actin filaments depends upon the type of protrusion: in lamellipodia, actin filaments form a branching dendritic network, while in filapodia, they form parallel bundles [9]. Actin polymerization is mediated by the Arp2/3 complex and the formins mDia1 and mDia2. The Arp $2 / 3$ complex binds to the side of the tip of a preexisting actin filament and promotes the formation of a new actin filament that branches off the mother filament [10]. Arp2/3 is regulated by WAVE/Scar, WASP and N-WASP proteins. WAVE/Scar belongs to a protein complex, and small Rho GTPase Rac can induce the dissociation of this complex to release and activate WASE/Scar .[11] In contrast, formins 
bind to the end of actin filaments and promote actin polymerization in a linear fashion. Formins are regulated by other small Rho GTPase (RhoA for mDial and Cdc42 for mDia2) [12]. Therefore, the small Rho GTPase family is pivotal for protrusion.

In order for a cell to migrate, a protrusion must be stabilized by attaching to the extracellular matrix (ECM) or adjacent cells. Integrins are the major receptors that serve as the feet to support the adhesion of migrating cell to its surroundings. Integrins comprise a large family of receptors on the cell surface, which are composed of $\alpha$ and $\beta$ subunits, and each combination of $\alpha$ and $\beta$ has its own binding specificity and signaling properties [13]. The binding of ligands to integrins either induces receptor clustering, which can increase the recruitment for effector molecules, or induces receptor conformational change, which can create or expose more binding sites to recruit effectors. These two ways lead to the assembly of signaling complexes at the cytoplasmic side of the plasma membrane, therefore regulating signal transduction [14]. For example, receptor clustering initiates protein tyrosine phosphorylation, small GTPase activation, to regulate the adhesion formation and maintenance during cell migration [15].

\subsubsection{Adhesion Disassembly and Retraction of the Cell Rear}

In order for cells to move forward, adhesions at the rear of migrating cells must disassemble. The contractile forces facilitating the retraction of the trailing edge are generated by actomyosin network reorganization $[16,17]$. Myosin II is a major regulator during this process as well as in the maintenance of polarity $[18,19]$. Cells deficient in myosin II show impaired rear retraction and the formation of multiple pseudopodia [2022]. Both myosin IIA and IIB are involved in this process but each has distinct contributions. Myosin IIA contributes to cell rear retraction and adhesion assembly, while myosin IIB is involved more in actin filaments crosslinking and the establishment of a front-rear polarity $[23,24]$.

In response to the actomyosin contractility, focal adhesion disassembly induces the separation of the cell rear from the substrates beneath. The interaction between trailing edge and substrates is maintained by integrins, and during retraction the integrins still attach to the substrates, while other components of the focal adhesion move forward with the cell body [25]. Additionally, ECM proteases could either digest the underlying substrates, or cleave cell surface proteins, therefore, disrupting adhesion and allowing retraction to occur [26].

\subsection{Principles of Focal Adhesions}

Focal adhesions (FAs) are the cell-substrate adhesion structures mediated by integrin. They anchor the ends of actin filaments and mediate strong attachment to substrates. FAs are dynamic, large protein complexes containing more than 100 proteins [27]. These protein components can be divided into extracellular ligands, transmembrane connector and cytoplasmic proteins according to their localization [28, 29]. The 
extracellular ligands are found in ECM as FA targets, such as collagen, fibronectin, heparin sulfate and laminin. The transmembrane proteins are mainly the integrin family members mediating interaction between the EMC and cytosol. The major components of FAs localized in the cytoplasmic region and can be further divided into three subgroups: 1) structural proteins, including components involved in the construction and movement of cytoskeleton, such as actin, $\alpha$-actin, and talin, 2) enzymatic proteins, including protein kinase, protein phosphatase and modulators of small GTPase, 3) adaptor proteins, which are multi-domain proteins mediating recruitment of other components to FAs, such as paxillin and p130Cas [28] (Figure 1-1).

\subsubsection{Cell Signaling Through FAs}

Cell-ECM attachment transmits extracellular signals from ECM into cytosol, which are important for cell growth, survival and migration. This process is mainly performed by integrin family members and contributes to the formation of the FAs. Integrins have no enzymatic activity; however, integrin clustering induces the phosphorylation of tyrosine residues in various proteins of FAs, one of which is focal adhesion kinase (FAK). Phosphorylated FAK can then induce the phosphorylation of other downstream enzymatic and scaffold proteins, transmitting signals from EMC to the nucleus [30].

FAs can transduce signals through the cell membrane in either "inside-out" or "outside-in" patterns. In addition, FAs act as " mechanosensors" as well as collaborate with various growth factor signaling pathways to perform their physiological functions [31]. The "inside-out" signaling means the activation of integrins is regulated form the inside of the cell. The activity of integrins modulates the affinity between cells and ECM, determining the migration status of the cell. Proteins in the integrin family possess a large extracellular domain and a small intracellular tail. Under the inactivated condition, the large extracellular domain of integrins is in a bent conformation corresponding to the lack of activity. Under the "inside-out" activation, the intracellular FA protein talin binds to the cytoplasmic tail of integrins, resulting in the separation of $\alpha$ and $\beta$ subunits. The separation of the intracellular domains leads to a distant transmembrane conformational change, which induces the opening of the extracellular domain in a "switchblade" pattern. The integrins then expose a new binding interface and are completely activated. The exposed epitope is named as a ligand induced binding site (LIBS) [32, 33]. Upon "outside-in" activation, the cytoplasmic tails of integrins recruit many molecules on to the FAs, including FAK, Src family kinase (SFK) and integrin-linked kinase (ILK). Briefly, the integrin $\beta 3$ tail binds talin, which recruits FAK into FAs. Upon transautophosphorylation, FAK is activated and functions as a scaffold protein to bring in SFK, activated phosphatidylinositol 3 kinase (PI3K) and extracellular signal regulated kinase (ERK)/ mitogen activated protein kinase (MAPK). In addition, FAK activates Rho family small GTPases, including Rac1, Cdc42, and RhoA, controlling the cytoskeleton formation and myosin contractility to regulate cell adhesion and migration. The RacRelA/NF- $\kappa$ B, PI3K-Akt/Ras-ERK linkages also influences gene transcription, promoting 


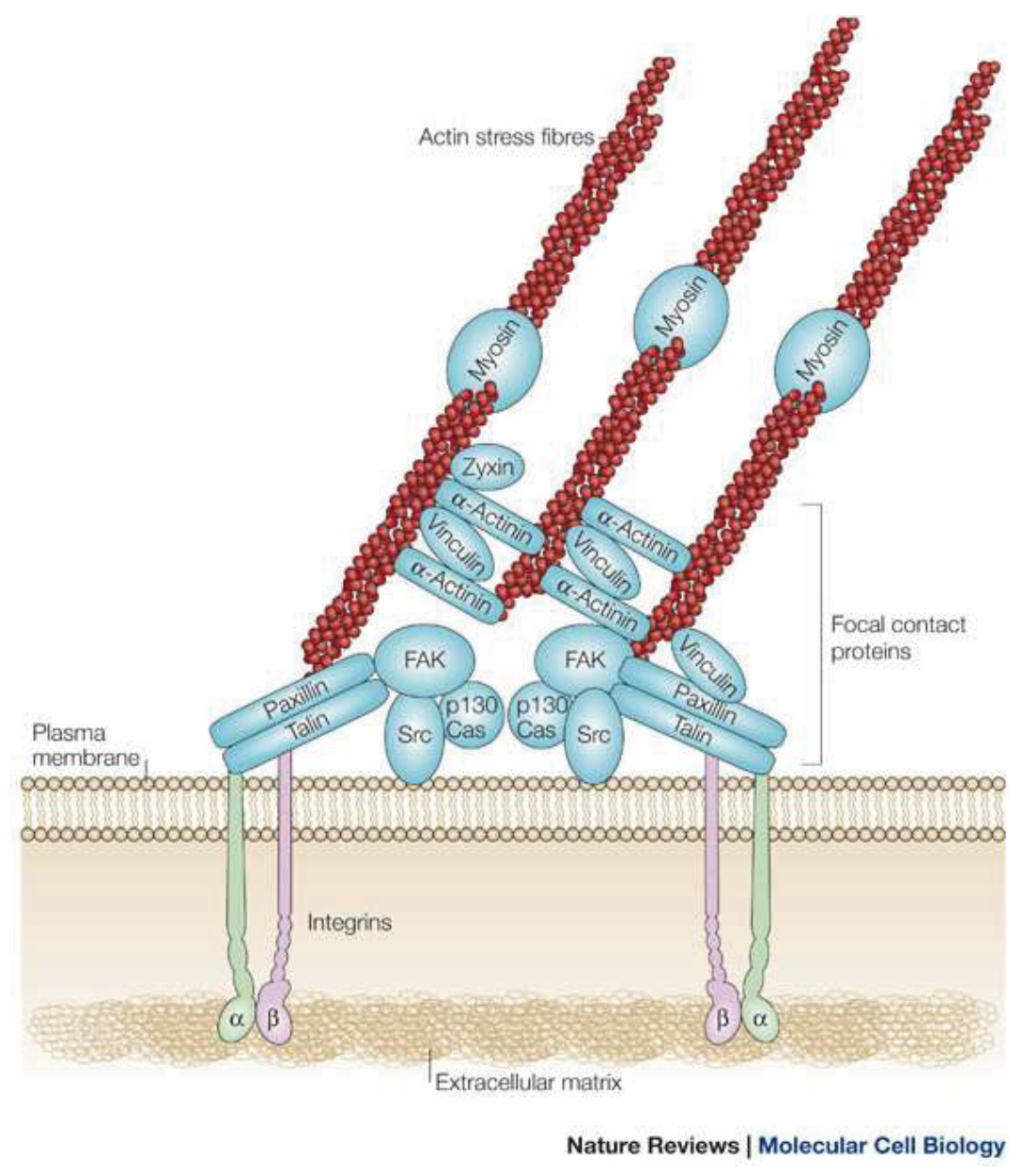

Figure 1-1. Molecular architecture of focal contacts.

The extracellular matrix, integrins ( $\alpha$ - and $\beta$-transmembrane heterodimeric proteins) and the cell cytoskeleton interact at sites called focal contacts. Focal contacts are dynamic groups of structural and regulatory proteins that transduce external signals to the cell interior and can also relay intracellular signals to generate an activated integrin state at the cell surface. The integrin-binding proteins paxillin and talin recruit focal adhesion kinase (FAK) and vinculin to focal contacts (see figure). $\alpha$-Actinin is a cytoskeletal protein that is phosphorylated by FAK, binds to vinculin and crosslinks actomyosin stress fibres and tethers them to focal contacts. Zyxin is an $\alpha$-actinin- and stress-fibre-binding protein that is present in mature contacts. Although the aforementioned proteins are found in most focal contacts, the membrane-associated protein tyrosine kinase Src and the adaptor protein p130Cas associate with focal contacts following integrin clustering. Integrin-mediated FAK activation is mediated in part by matrix binding or by forcedependent changes in cytoskeletal linkages. Several other proteins such as extracellular signal-regulated kinase 2 (ERK2) and calpain are known to be transiently present at focal contacts (not shown). The composition of a focal contact is therefore constantly varying depending on external cues and cellular responses. Reprinted with permission from Macmillan Publishers Ltd: Mitra, S.K., D.A. Hanson, and D.D. Schlaepfer, Focal adhesion kinase: in command and control of cell motility. Nat Rev Mol Cell Biol, 2005. 6(1): p. 56-68. 
cell survival and proliferation [31]. On the other hand, the signaling also plays a role in disassembly of FAs at the trailing edge. For example, Rac can either directly through its effector p21-activated kinase (PAK), and/or indirectly through antagonizing Rho induce the detachment. Other mechanism involves the recruitment of PAK by GIT into the FAs. Activated PAK can catalyze the disassembly of actin stress fibers and has been found to be the central player for FAs disassembly [34, 35].

The "outside-in" signaling means the binding of EMC to the extracellular domain of integrins elicits signals that are transmitted into the cell. This is the classical function of FAs as environmental and chemical sensors, allowing the delivery of different information into the cell to induce corresponding responses. Interaction between integrins and ECM components induces the same conformational change during " outside-in " signaling, stabilizes integrins in an activated form, and facilitates the interaction between the integrins cytoplasmic tail and signaling and structural proteins in FA complex [31].

FAs are also necessary for the optimal activation of growth factor receptors as they can only be optimally activated under appropriate cell attachment conditions. It has been revealed that FAs signaling is required for the potent efficient signal transduction induced by the epidermal growth factor receptor (EGFR), the vascular endothelial growth factor receptor (VEGFR) and the platelet-derived growth factor receptor (PDGFR). The association of $\beta 3$ integrin with those growth factor receptors is critical for their functions [36-38]. It is well elucidated that FAs signaling shares two major downstream pathways with the growth factor signaling: the Ras/Raf-ERK/MAPK pathway and the PI3K-Akt pathway [39]. Both of them are pivotal for apoptotic cell death and cell proliferation. The activated ERK/MAPK promotes survival either through regulating the activity and level of BCL-2 family members in the intrinsic apoptosis pathway such as anti-apoptotic BCL2 and pro-apoptotic BAX [40], or via altering the level of transcription factor p53 to regulate the cell cycle [41]. On the other hand, the activated PI3K-Akt pathway suppresses apoptosis by activating Mdm2 or inactivating BAD, Caspase9 or forkhead transcription factor (FKHR) [42]. It also promotes cell growth and proliferation by regulating the mTOR pathway and cell cycle $[42,43]$.

\subsubsection{Key Components of FAs}

\subsubsection{Focal adhesion kinase}

The focal adhesion kinase (FAK) is a ubiquitously expressed non-receptor and non-membrane associated protein tyrosine kinase (PTK). It was identified in 1992 and shown to localize to FAs as one of the most crucial "integrators" for signal transduction $[44,45]$. FAK is comprised of three main domains: a N-terminal FERM (protein 4.1, ezrin, radixin and moesin homology) domain, a central kinase domain, and a C-terminal focal adhesion-targeting (FAT) domain [46] (Figure 1-2). The FERM domain is known to mediate protein-protein interaction and protein-membrane interaction [47]. The FERM domain can interact with the cytoplasmic tails of $\beta$-intergins, and various growth 


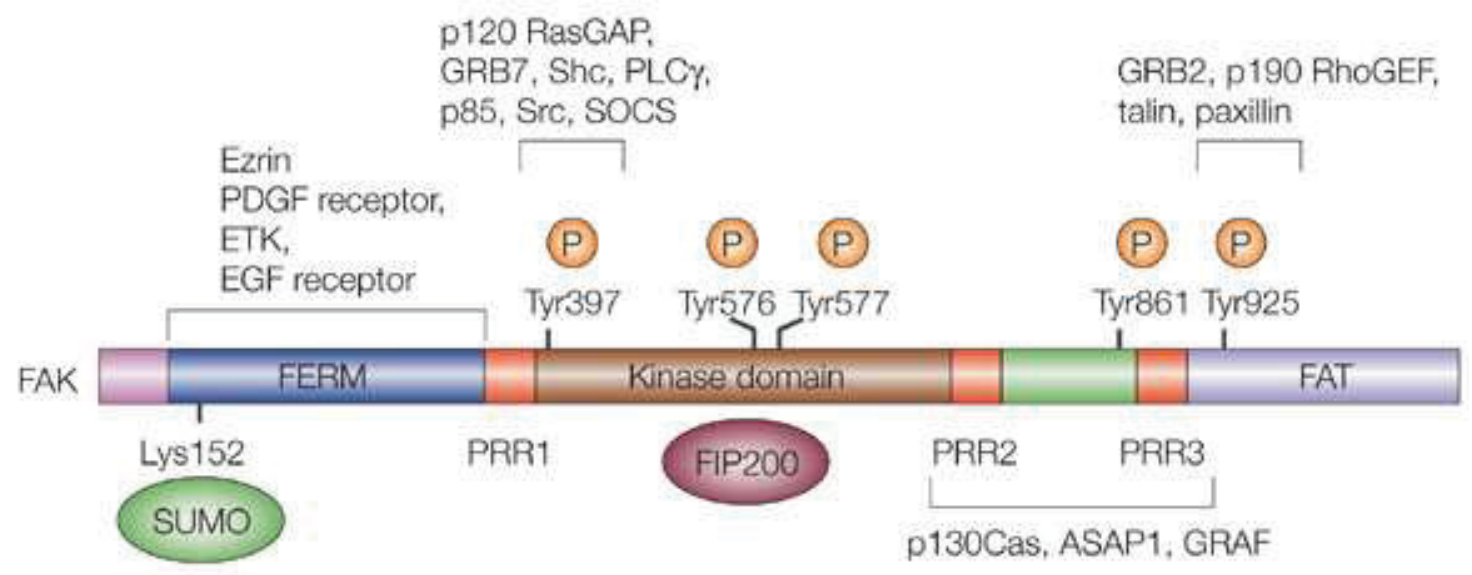

Nature Reviews | Molecular Cell Biology

Figure 1-2. Focal adhesion kinase domain structure and phosphorylation sites. Focal adhesion kinase (FAK) contains a FERM (protein 4.1, ezrin, radixin and moesin homology) domain, a kinase domain and a focal adhesion targeting (FAT) domain. The FERM domain mediates interactions of FAK with the epidermal growth factor (EGF) receptor, platelet-derived growth factor (PDGF) receptor, the ETK tyrosine kinase and ezrin, and the FERM domain can be conjugated to SUMO (small ubiquitin-related modifier) at Lys 152. The FAT domain recruits FAK to focal contacts by associating with integrin-associated proteins such as talin and paxillin. It also links FAK to the activation of Rho GTPases by binding to guanine nucleotide-exchange factors (GEFs) such as p190 RhoGEF. FAK contains three proline-rich regions (PRR1-3), which bind Src-homology3 (SH3) domain-containing proteins such as p130Cas, the GTPase regulator associated with FAK (GRAF) and the Arf-GTPase-activating protein ASAP1. FAK is phosphorylated (P) on several tyrosine residues, including Tyr397, 407, 576, 577, 861 and 925. Tyrosine phosphorylation on Tyr397 creates a Src-homology-2 (SH2) binding site for Src, phospholipase Cgamma (PLCgamma), suppressor of cytokine signalling (SOCS), growth-factor-receptor-bound protein 7 (GRB7), the Shc adaptor protein, p120 RasGAP and the p85 subunit of phosphatidylinositol 3-kinase (PI3K). Phosphorylation of Tyr576 and Tyr577 within the kinase domain is required for maximal FAK catalytic activity, whereas the binding of FAK-family interacting protein of $200 \mathrm{kDa}$ (FIP200) to the kinase region inhibits FAK catalytic activity. FAK phosphorylation at Tyr925 creates a binding site for GRB2. Reprinted with permission from Macmillan Publishers Ltd: Mitra, S.K., D.A. Hanson, and D.D. Schlaepfer, Focal adhesion kinase: in command and control of cell motility. Nat Rev Mol Cell Biol, 2005. 6(1): p. 56-68. 
factor receptors, such as epidermal growth factor (EGF) receptor, platelet-derived growth factor (PDGF) receptor, to promote cell motility and mediate signal transduction $[48,49]$. Published work reveals that truncation of the FERM domain leads to enhanced FAK phosphorylation and activation, indicating an inhibitory role of FERM in FAK activation $[50,51]$. This autoinhibition is due to the interaction between the FERM domain and the kinase domain, blocking access to the catalytic cleft and preventing the FAK activation loop from phosphorylation [52]. The central kinase domain is essential for the tyrosine phosphorylation of FAK itself (autophosphorylation) and its downstream targets [53]. Autophosphorylation of FAK at Tyr397 enables it to be recognized by various Srchomology (SH) 2-domain-containing proteins, including SFKs, and Scr-mediated FAK phosphorylation at Tyr 576 and Tyr577 within the kinase domain activation loop promotes maximal FAK catalytic activation [54]. In addition, the recruitment of SFKs facilitates the phosphorylation of various FAK-associated proteins, including paxillin and p130Cas [54-56]. FAT at the C-terminus of FAK is a protein-protein interaction domain, consisting of four alpha helixes forming a helical bundle [30], which is the binding site for paxillin and talin $[57,58]$. The FAT domain directs FAK to FAs, and fusion of the FAT domain with other proteins is sufficient to drive the FAs localization $[59,60]$. The C-terminus of FAK also possesses two proline-rich regions (PRRs) as binding sites for Src homology 3 (SH3) domain containing proteins (such as p130Cas) [54, 55]. The protein-protein interaction is important in regulating cytoskeletal dynamics and FAs assembly, and promoting cell migration [58].

Numerous studies have indicated that elevated FAK expression and enhanced activity is associated with malignancy of various tumor cells, including neuroblastoma, melanoma, and colon cancer [61]. In tumor cells, decreasing of FAK expression induces cell detachement and apoptosis $[62,63]$. Studies have revealed that overexpression of wild-type FAK in tumor cells increases cell proliferation, while knocking-down FAK by shRNA induces apoptosis and decreases cell proliferation [63-65]. Given the role of FAK in tumorgenesis and malignancy, it could be a promising target for cancer therapy. TAE226, a small molecule inhibitor targeting FAK, can improve survival of tumorloaded animals in vivo, and disrupt glioma cell proliferation, attachment, migration and invasion in vitro [66]. In addition, this compound also induces breast cancer cell apoptosis in vitro [67]. Another small molecular FAK inhibitor, PF-652.271 is able to inhibit tumor growth and angiogenesis[68]. Thus, targeting FAK provides a prospective approach for cancer therapy.

\subsubsection{Paxillin}

Paxillin belongs to the paxillin family that includes paxillin, Hic-5 and leupaxin, a leukocyte-specific form (Figure 1-3). Paxillin was originally identified in Rous sarcoma virus-transformed fibroblasts as a substrate for non-receptor tyrosine kinase $p p 60 v-S r c$ [56]. Paxillin is a scaffold protein in FAs and functions as a signaling hub by recruiting other proteins to integrate and disseminate signals from integrins and other growth factor receptors. In addition, paxillin also participates in the FAs-cytoskeleton connections through the interaction with actin binding protein vinculin and actopaxin[69]. 


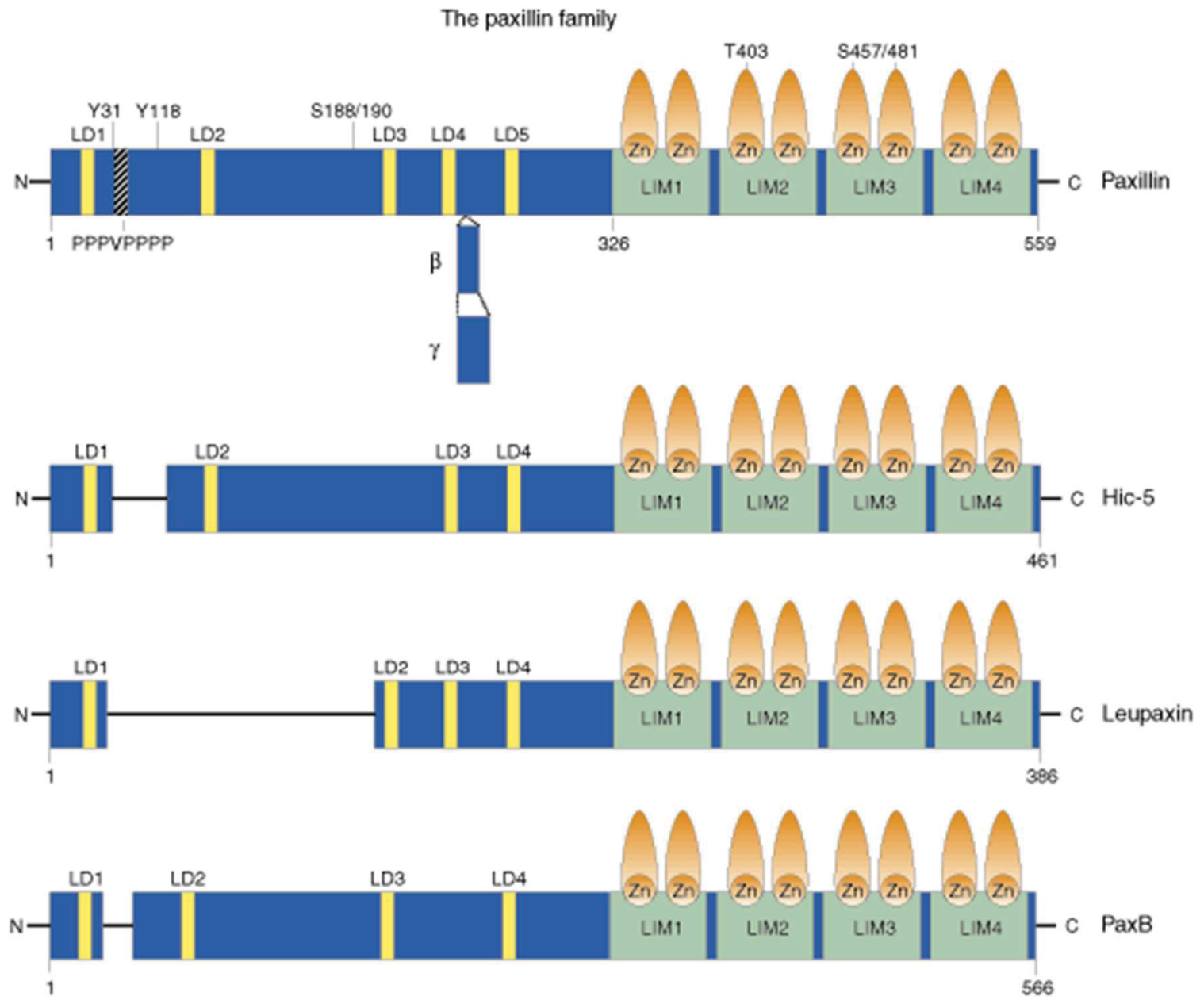

Figure 1-3. The paxillin family and domain structure.

Paxillin is highly conserved between species ( $90 \%$ identity between chicken and humans). Alternative splice variants ( $\beta$ and $\gamma$ ) have been identified in humans and mouse. Paxillin-related proteins such as Hic5, leupaxin and PaxB share extensive homology with paxillin in their LIM domains and LD motifs. These variants lack the putative Src SH3binding proline-rich sequence and also key phosphorylation sites, suggesting that these proteins may complement some of paxillin's functions while antagonizing others. Reprinted with permission from Macmillan Publishers Ltd: Turner, C.E., Paxillin and focal adhesion signalling. Nat Cell Biol, 2000. 2(12): p. E231-6. 


\subsection{Paxillin structure}

As a scaffold protein, paxillin possesses many protein-binding modules, including the C-terminal LIM (Lin11, Isl-1, Mec-3) domains, N-terminal LD motifs, and multiple tyrosine, and Ser/Thr phosphorylation sites [56].

Paxillin contains four LIM domains at its C-terminal half (LIM1-LIM4). LIM domains are double-zinc-finger motifs that mediate protein-protein binding $[70,71]$. The LIM2 and LIM3 domains of paxillin are essential for anchoring the protein at the plasma membrane [72], possibly through interacting with the cytoplasmic tails of integrins [73]. Additionally, the phosphorylation of LIM2 and LIM3 domains contributes to the localization of paxillin to FAs [74]. Moreover, the LIM domains of paxillin have multiple binding partners, including tublin and tyrosine phosphatase PTP-PEST, and these interactions are involved in focal adhesion dynamics [75-79] (Figure 1-4).

The N-terminus of paxillin contains five leucine- and aspartate- rich motifs (LD1LD5). LD motifs were originally identified as the binding sites for vinculin and FAK [72, 80]. These protein-binding modules share the consensus sequence LDxLLxxL [81], which is highly conserved in paxillin from different species, and between paxillin and the other paxillin family members [82]. Surprisingly, despite the sequence conservation, individual LD motifs have their specific binding partners [56, 72, 79, 82] (Figure 1-4), and each member in the paxillin family also has its own unique set of interactions [8385]. NMR studies have revealed that LD peptides form amphipathic $\alpha$-helices, with the leucines providing a hydrophobic interface with its binding partners [86].

\subsection{Paxillin phosphorylation}

Paxillin possesses multiple tyrosine, serine, and threonine phosphorylation sites, which are targeted by a variety of kinases in the presence of adhesion stimuli and growth factors. A diverse array of kinases have been reported to induce paxillin phosphorylation, including Abl, FAK-Src, ERK, Jun N-terminal kinase (JNK), p38 mitogen-activated protein kinase (p38 MAPK), cyclin-dependent kinase 5 (CKD5), receptor for activated C kinase 1 (RACK1), and PAK [87]. j

It is clear that a variety of stimuli that signal through a range of transmembrane receptor families are able to induce paxillin tyrosine phosphorylation. Signaling through receptor tyrosine kinase ligands such as insulin-like growth factor I (IGF-I), growth hormone, epidermal growth factor (EGF), platelet-derived growth factor (PDGF), and vascular endothelial growth factor (VEGF) [88-92], agonists of 7-pass transmembrane family receptors such as epinephrine and angiotensin II [93, 94], and immunomodulators such as T-cell receptor and $\operatorname{IgE}[95,96]$, can induce paxillin tyrosine phosphorylation. The tyrosine phosphorylation of paxillin leads to the generation of functional SH2binding domains for protein interaction. The best-known SH-2 association is with the adaptor protein Crk, which bind to phosphorylated Y31 and Y118 [97]. Other SH-2 binding proteins are p85 PI3K binding to phosphorylated Y31, Y40 and Y118, Y31 


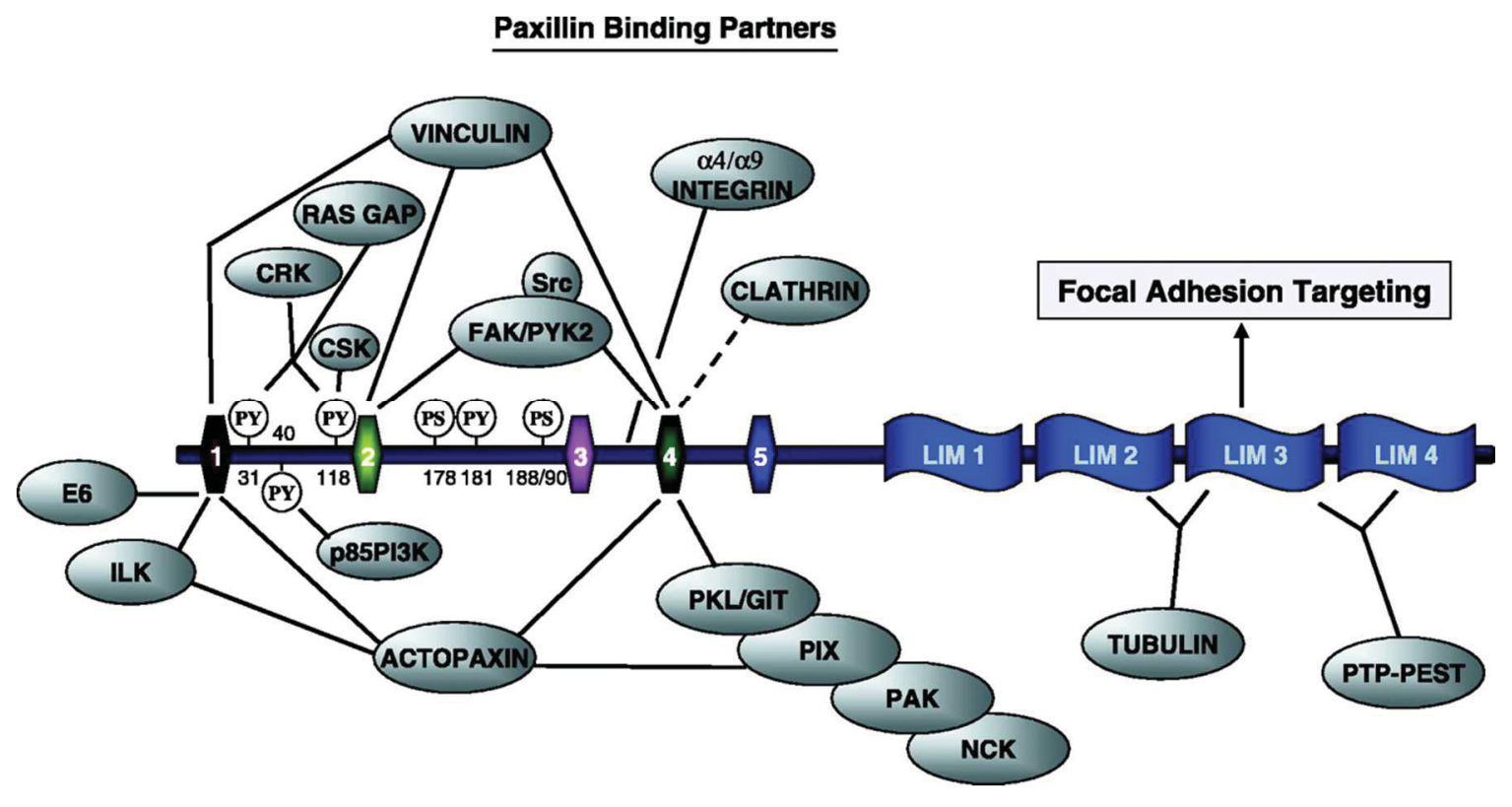

Figure 1-4. Paxillin binding partners.

Paxillin is comprised of multiple protein binding motifs, including the amino-terminal LD motifs, the carboxy-terminal LIM domains, and several phosphotyrosine-SH2 domain-docking sites. These individual motifs have been used in various biochemical GST-pull down, blot overlay assays, and yeast two-hybrid screens to identify numerous direct binding proteins. These range from structural actin binding proteins including actopaxin and vinculin to important signaling molecules such as FAK (focal adhesion kinase), ILK (integrin-linked kinase), and PTP-PEST, a tyrosine phosphatase. Many other potential paxillin binding partners have been identified in co-precipitation experiments, although in many of these cases it remains to be determined whether the interaction with paxillin is direct or which domain of paxillin is involved. Reprinted with permission from The American Physiological Society: Brown, M.C. and C.E. Turner, Paxillin: adapting to change. Physiol Rev, 2004. 84(4): p. 1315-39. 
and Y118 [97, 98] (Figure 1-5). The role of paxillin tyrosine phosphorylation will be discussed below.

In addition to tyrosine phosphorylation, paxillin serine/threonine phosphorylation is also reported. For example, paxillin S188/190 are phosphorylated after cellular activation by integrin ligation [99, 100]. Serine and threonine residues in LIM2 and LIM3 domains are also phosphorylated after adhesion and stimulation with growth factors such as angiotensin II, EGF and TGF- $\beta[101,102]$. Moreover, paxillin undergoes serine/threonine phosphorylation at the onset of mitosis [103]. Several kinases have been implicated in the serine/threonine phosphorylation process, including protein kinase $\mathrm{C}$ (PKC), PAK, IlK, ERK, MAPK [99, 104-107] (Figure 1-5).

On the other hand, Paxillin binding partners, tyrosine phosphatase PTP-PEST and serine/threonine phosphatase PP2A, mediate dephosphorylation of paxillin $[75,108]$. Disruption of paxillin and PTP-PEST interaction leads to decreased cell adhesion and motility, indicating a role of paxillin binding to PTP-PEST in regulating adhesion dynamics [75, 109]. Cells expressing an enzyme-dead form of PP2A show enhanced cell spreading and motility, suggesting that serine phosphorylation of paxillin is involved in these processes [110].

\subsection{Paxillin function and its implications}

As described in the previous section, Rho small GTPase family, which includes the proteins $\mathrm{Cdc} 42$, Rac and RhoA, are pivotal for cell migration. This family is undergoing dynamic switching by cycling between an inactive GDP-bound and active GTP-bound state. The cycling is controlled by guanine-nucleotide-exchange factors (GEFs), which catalyze the conversion from GDP to GTP, and by GTPase-activating proteins (GAPs), which promote the hydrolysis of GTP to GDP [111]. Paxillin can regulate the Rho small GTPase family by directly or indirectly recruiting a variety of GEFs, GAPs and other effector proteins to the FAs. Paxillin can be phosphorylated at tyrosine Y31 and Y118 in a FAK and Src dependent manner, generating a binding site for the SH-2 domain of the adaptor protein Crk [97, 112]. Crk, together with DOCK (dedicator of cytokinesis) 180 and ELMO, form a complex to activate GTPase Rac by means of the GEF activity of DOCK180, therefore, promoting cell migration [113]. Y31 and Y118 phophorylated paxillin also regulates RhoA activity by competing with p190RasGAP for binding to p120RasGAP. p190RasGAP that is free from p120RasGAP can suppress RhoA activity, which is necessary for efficient membrane spreading and adhesion during cell migration [97]. The Paxillin LD4 domain is also important for the regulation of Rho GTPase signaling. This domain is capable of recruiting a complex composed of the G-protein-coupled receptor kinase interacting protein (GIT1/2), PAKineracting exchanger factor (PIX), PAK and non catalytic region of tyrosine kinase adaptor protein (NCK) to the leading edge of migrating cells [34, 114, 115]. GIT1/2 belongs to ArfGAP family and can indirectly stimulate Rac activity [116]. PIX possesses Rac and Cdc42 GEF activity [117]. Paxillin mutant that lacks the LD4 motif cannot recruit the GIT-PIX-PAK-NCK complex to focal adhesion. Fibroblasts with this mutant 
Paxillin Phosphorylation and Function

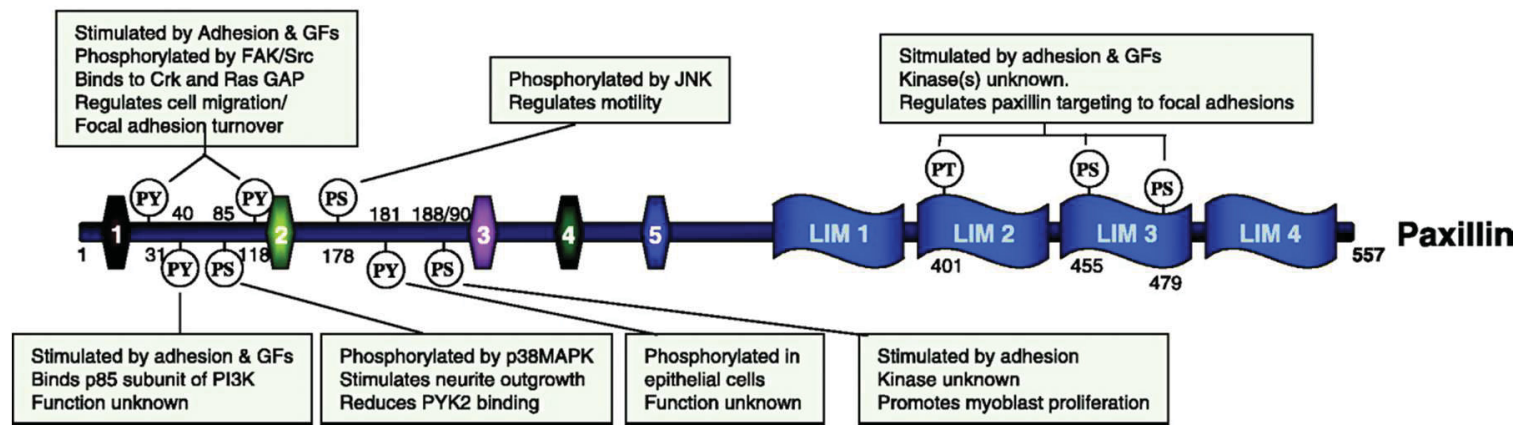

Figure 1-5. Paxillin phosphorylation and function.

Paxillin is phosphorylated on multiple tyrosine (PY), serine (PS) and threonine (PT) residues in response to cell adhesion, and/or exposure to a various soluble growth factors and cytokines (see text for details). Tyrosine phosphorylation generates docking sites for SH2 domain containing proteins such as Crk (binds PY31, 118) to facilitate downstream signaling. In contrast, phosphorylation of serine and threonine residues is more likely to influence paxillin conformation and thereby allosterically affect its ability to interact with specific binding partners. Reprinted with permission from The American Physiological Society: Brown, M.C. and C.E. Turner, Paxillin: adapting to change. Physiol Rev, 2004. 84(4): p. 1315-39. 
exhibit abnormal polarization and protrusion in the process of cell migration $[79,114]$.

Paxillin has been reported to regulate cell survival. Anti-apoptotic BCL-2 can interact with the paxillin LD4 domian and facilitate kidney development[84]. Further structure analysis reveals that BCL-2 possesses an amphipathic helical-bundle-like topology similar to the LD-motif-binding protein vinculin, GIT1 and FAK [118-121]. The interaction of vinculin with paxillin LD1 and LD2 motifs competes with FAK for paxillin binding, and thereby promotes ERK signaling to prevent apoptosis $[115,122]$. Additionally, paxillin has been identified as a Caspase-3 substrate and can be cleaved at six different aspartic acid residues, which inhibit integrin-mediated cell survival signals and promote apoptosis [123].

Genetic ablation of the paxillin gene is embryonic lethal due to abnormal adhesion dynamics and cell migration, indicating that this protein is crucial for embryonic development [124]. Given the role of paxillin in regulating cell migration and survival, it has also been linked to tumor malignancy and metastasis. For example, a number of paxillin gene sequence aberrations located between LD1 and LD2 motifs have been observed in lung adenocarcinoma samples, which are implicated in tumor growth and invasion $[125,126]$. Moreover, phosphorylation of paxillin downstream of EGF, PDGF, TGF- $\beta$, and androgen receptor activation has been observed in a variety of malignancies and affects tumorigenesis and metastasis [127-130]. Therefore, expression and phosphorylation of paxillin has been implicated as a prognostic marker for patients suffering from breast cancer, hepatocellular cancer, lung adenocarcinoma, and ovarian cancer [131-135]. Meanwhile, paxillin is attractive target for anti-metastatic drug development.

\subsubsection{P130Cas}

The p130Cas (CAS-associated substrate) is a Src substrate colocalized with paxillin as a component of the FAs, which is involved in cell migration, survival, and transformation, invasion and caner [136]. p130Cas was first identified as a prominent tyrosine-phosphorylated protein in cells that are transformed by v-crk and v-src [137, 138]. P130Cas is not an enzymatic protein but a docking and scaffolding protein [139], which can either mediate protein-protein interactions or directly interact with other proteins through its various domains and motifs. For example, FAK is a key component of FAs that interacts with p130Cas [140]. It is well established SH3 domains of p130Cas interacts with FAK through its N-terminal FERM domain. Similar to FAK, the p130Cas localizes to FAs and is phosphorylated by tyrosine kinase in response to adhesion signals [141-143]. A primary role of p130Cas in FA signal transduction is a signaling component that brings cellular responses to integrin engagement [144, 145]. p130Cas has been demonstrated to be responsible for tamoxifen resistance in estrogen receptor positive breast cancer [146]. Therefore, the human homolog of p130Cas is known as BCAR1 (product of breast cancer anti-estrogen resistance gene 1) as well. The mechanism of antiestrogen resistance studies provide solid evidence that p130Cas plays an important role in promoting cell proliferation and survival [147-150]. 


\subsection{Cas-family members}

p130Cas belongs to the Cas family, which consists of four proteins: p130Cas, neural precursor cell expressed, developmentally down-regulated 9 (NEDD9, also known as HEF1 or Cas-L), embryonal Fyn-associated substrate (EFS, also known as Sin), and Cas scaffolding protein family member 4 (CASS4, also known as HEPL) [151]. Cas family members share homology especially in the N-terminal SH3 domain and the Cterminal Cas-family C-terminal Homology domain (CCHD) $[145,152]$. Despite the homology within Cas family members, p130Cas has non-redundant roles compared to the other family members, as only genetic ablation of p130Cas gene results in embryonic lethality at E12.5 due to cardiovascular defects [152].

\subsection{P130Cas structure and binding proteins}

As a scaffolding protein, p130Cas contains several protein-binding motifs, including an N-terminal SH3 domain, a substrate domain (SD) containing SH2 binding motifs, a serine-rich (SER) domain, a Src-binding domain (SBD), and a Cas-family Cterminal homology domain (CCHD) [136] (Figure 1-6). The structure and binding partners of these domains will be discussed in detail below.

1.2.2.3.2.1 The Scr-homology 3 domain and binding proteins. P130Cas has an $\mathrm{N}$-terminal SH3 domain, which mediates the interaction with proteins containing prolinerich motifs [152]. The SH3 domain is known to interact with FAK [140, 153], FAK family member protein tyrosine kinase 2 (PYK2) [154], GEF C3G [155], Cas-ligand with multiple SH3 domains (CMS) [156], antimicrobial peptide PR-39 [157], protein tyrosine phosphatase PTP1B and PTP-PEST [158, 159], CIZ [160] and focal adhesion kinaserelated nonkinase (FRNK) [161]. The p130Cas SH3 domain binds to two PPRs at the Cterminus of FAK [54, 55, 140]. Despite the direct interaction between p130Cas and FAK, SFKs, rather than FAK, contribute to the phosphorylation of p130Cas. In this scenario, FAK serves as a scaffold protein to recruit Src to p130Cas via the interaction between the Src SH2 domain and the FAK autophosphorylation site Y397 [54, 140].

The PYK2 [or the related adhesion focal tyrosine kinase (RAFTK)] is tyrosine phosphorylated and complexes with p130Cas in cells upon the ligation of $\beta 1$ integrin and B cell antigen receptor (BCR). PYK2 can directly phosphorylate the substrate domain of p130Cas. However, the phosphorylation of the C-terminus of p130Cas is dependent upon an intact autophosphorylation site Y402 on PYK2, which mediates the binding of PYK2 to c-Src kinase, indicating that cooperation between PYK2 and c-Src is necessary for the complete phosphorylation of $\mathrm{p} 130 \mathrm{Cas}[154,162]$. The direct interaction of p130Cas and C3G was identified by the two-hybrid system. Distinct from the well-documented Crk binding sites in the central region of $\mathrm{C} 3 \mathrm{G}$, the proline-rich motif $\left(\mathrm{Ala}^{0}-\mathrm{Pro}^{1}-\mathrm{Pro}^{2}-\mathrm{Lys}^{3}\right.$ $\mathrm{Pro}^{4}-\mathrm{Pro}^{5}-\mathrm{Leu}^{6}-\mathrm{Pro}^{7}$ ) in the N-terminal region of $\mathrm{C} 3 \mathrm{G}$ mediates the binding of the p130Cas SH3 domain. Mutagenesis studies revealed that $\mathrm{Pro}^{1}, \mathrm{Lys}^{3}$, and $\mathrm{Pro}^{4}$ are crucial for the protein-protein interaction [155]. C3G is a guanine exchange factor, and has been 


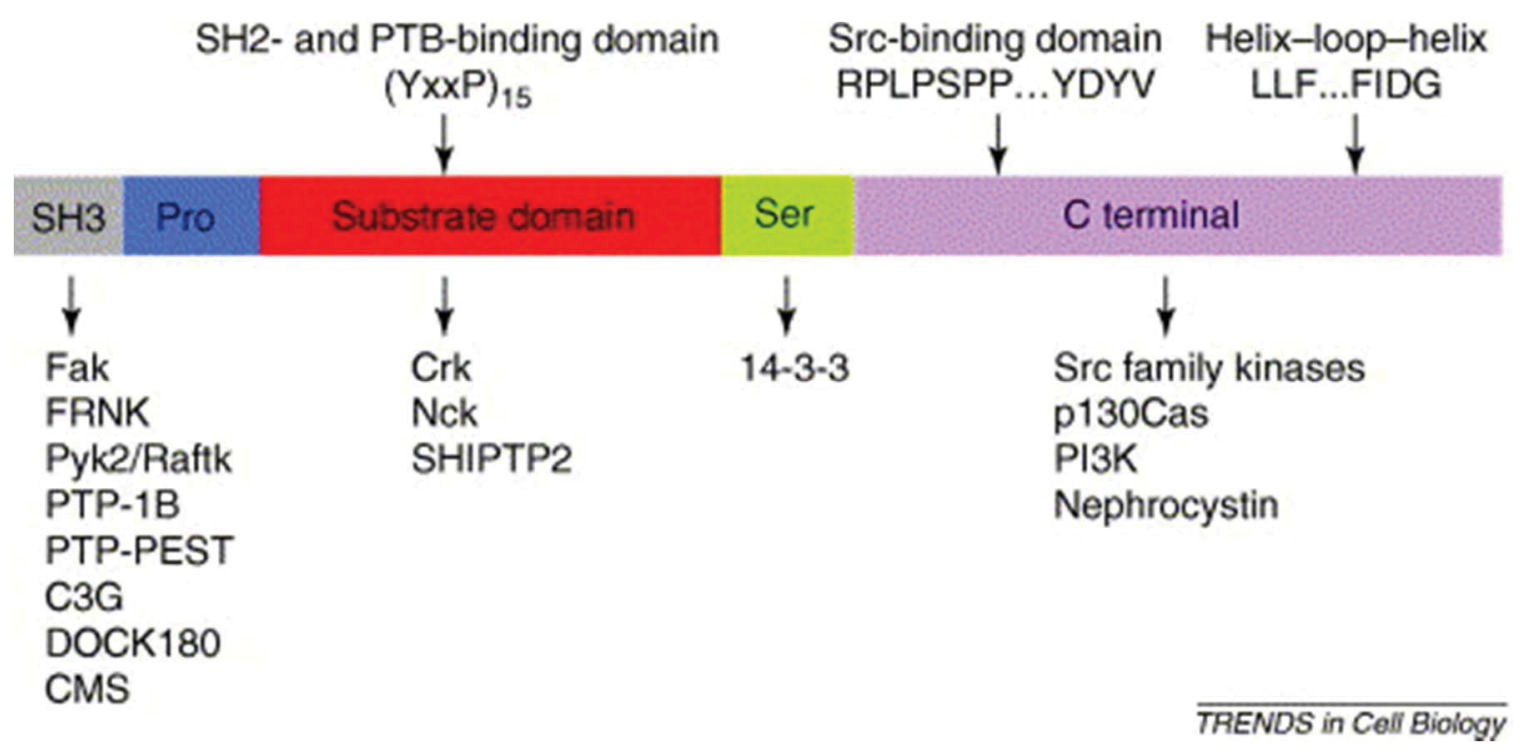

Figure 1-6. The structural characteristics and interacting proteins of p130Cas.

P130Cas is a scaffold molecule containing: (i) an N-terminal SH3 domain; (ii) a prolinerich region; (iii) a large substrate-binding domain containing 15 repeats of a YxxP sequence; (iv) a serine-rich region; and (v) a C-terminal domain. The tyrosine residues in the YxxP sequences are a substrate of PTK and, when phosphorylated, provide a binding site for the $\mathrm{SH} 2$ or PTB domains of effector proteins. The C-terminal domain is characterized by a bipartite binding site that includes a proline-rich region (RPLPSPP), which binds to the Src SH3 domain, and a tyrosine-containing sequence (YDYV), which binds to the Src SH2 domain when phosphorylated. A HLH motif is also present in this region. Proteins that directly bind to p130Cas domains are listed. Proteins that associate with p130Cas indirectly include PR-39, Grb2, zyxin, LPP, TRIP-6, Ajuba, p140Cap, Bmx/Etx and NPM-ALK. Reprinted with permission from Elsevier B. V.: Defilippi, P., P. Di Stefano, and S. Cabodi, p130Cas: a versatile scaffold in signaling networks. Trends Cell Biol, 2006. 16(5): p. 257-63. 
related to Rap1, R-Ras, and R-Ras2 [163]. The role of C3G in p130Cas signaling still needs to be elucidated.

CMS is an adaptor protein, composed of three N-terminal SH3 domains, followed by a PPR, a 160-residue unstructured region, and a C-terminal coil-coil domain [156]. The PPR is the site for binding of SH3 domain containing proteins, such as p130Cas. Other interacting partners include Src-family kinase, PI3K, and Grb, et al [156]. CMS has been shown to localize to dynamic actin at the leading edge of cells and membrane ruffles [156]. CMS-deficient mice die of kidney failure within the age of six to seven weeks due to a defect in the podocytes actin-rich foot processes [164], suggesting that CMS serves as an adaptor protein for the regulation of the actin cytoskeleton.

PR39 is a proline-arginine-rich antimicrobial peptide. It has multiple functions, including LPS neutralization, wound healing, inhibiting bacteria DNA and protein synthesis, affecting cell motility and metastatic potential. PR39 is capable of entering cells and interacts with the SH3 domain of p130Cas via its PPR, mainly in the cytoskeletal fraction. Using a biologically active fragment of PR-39 to treat cells results in a decrease in cytosolic p130Cas level, whereas an increase in cytoskeletal p130Cas in a dose-dependent manner. These data suggest that PR-39 can enhance p130Cas cytoskeleton association to activate p130Cas signaling pathways [157].

PTP1B is a protein tyrosine phosphatase, interacting with the p130 Cas SH3 domain via one of its first PPR (amino acids 301-315). The interaction between these two proteins is independent of p130Cas tyrosine phosphorylation, but can be abolished by replacing two critical proline residues (proline 309 and 310) in the first PPR [165]. PTP1B has been implicated in the regulation of integrin signaling, however, its actual role in this process is quite controversial among different studies. PTP1B is initially reported in overexpression studies to negatively regulate integrin signaling via dephosphorylation of p130Cas $[159,165]$. In a separate study, PTP1B is proposed to positively regulate integrin signaling, as SV40 transformed embryonic fibroblasts derived from PTP1B knockout mice exhibit reduced p130Cas phosphorylation and cell spreading [166]. Similar phenotype has been observed in another study by using small molecules to target PTP1B [167]. These discrepancies are possibly due to overexpression and knockout/knockdown strategies employed in each study.

PTP-PEST is a tyrosine phosphatase, with a highly restricted substrate preference; therefore, it is capable of choosing tyrosine-phosphorylated p130Cas as a substrate from a mixture of tyrosine phosphorylated proteins. The interaction between these two proteins is due to two distinct mechanisms. A PTP-PEST mutant deficient in catalytic activity but retaining the ability to bind substrates does not associate with p130Cas, indicating that the catalytic domain of PTP-PEST contributes to the specificity of binding to p130Cas. Mutation of proline 337 to alanine within the PTP-PEST C-terminal proline-rich region dramatically impairs the recognition of PTP-PEST to tyrosine phosphorylated p130Cas, without affecting the specificity of the interaction, suggesting that the $\mathrm{SH} 3$ domain of PTP-PEST mediates the association with p130Cas to increase the efficiency of the 
interaction $[158,168]$. Dephosphorylation of p130Cas leads to the termination of tyrosine-phosphorylation dependent signaling events downstream of p130Cas.

\subsection{The substrate domain and binding proteins. Tyrosine}

phosphorylation of p130Cas is mainly within the SD located in the N-terminal half of the protein $[139,169]$. The SD of p130Cas contains a large number of YxxP motifs, which when phosphorylated by Src-family kinases generates binding sites for proteins containing SH2 domains. Phosphorylation of SD by Src-family kinases is predominantly at FAs and integrin adhesion sites [170, 171]. SD phosphorylation can be enhanced by physical extension of the SD, indicating that p130Cas is a mechanosensor [172]. Utilizing p130Cas-deficient MEFs expressing variant mutants reveals that tyrosine phosphorylation is required for cell leading edge actin assembly and protrusion, and enhances FAs assembly and disassembly [173]. Moreover, tyrosine phosphorylation of p130Cas SD has been shown to be an important event in estrogen receptor-negative breast cancer cell migration, invasion and survival [174]. Crk and Nck have been reported to associate with phosphorylated SD of p130Cas [152]. Crk interacts with the last ten YxxP motifs of SD, while Nck associates with p130Cas within the first six YxxP motifs $[175,176]$. Crk, together with DOCK (dedicator of cytokinesis) 180 and ELMO, form a complex to activate GTPase Rac by means of the GEF activity of DOCK180, therefore, promoting cell migration [113]. The SH2 domain-dependent binding of Nck to p130Cas SD facilitates signaling to ERK2, enhancing cell proliferation, cell cycle progression, and cell migration $[175,176]$.

1.2.2.3.2.3 The serine-rich domain and binding proteins. Besides the tyrosine phosphorylation sites, p130 Cas also possesses a serine-rich domain (SER). Structure analysis reveals that the SER domain folds into a four-helix bundle, which bears structural similarity to other adhesion component such as FAK, $\alpha$-catenin, or vinculin[177]. This domain serves as a protein-interaction motif, and its binding partners include the 14-3-3 protein $[177,178]$. Deletion of this domain significantly reduces the ability of p130Cas to bind 14-3-3 protein. The interaction between SER and the 14-3-3 protein is serine phosphorylation dependent. Structure analysis of the 14-3-3 protein identifies that lysine 49 is lining its amphipathic peptide-binding groove; which mediates electrostatic interaction with phosphorylated serine in 14-3-3 protein binding partners. Mutation of lysine49 in the 14-3-3 protein to aspartic acid can completely abolish its interaction with p130Cas. Alkaline serine phosphatase treatment greatly reduces p130Cas and 14-3-3 protein interaction. Furthermore, the p130Cas and 14-3-3 protein interaction is regulated by integrin signaling. The binding of $\mathrm{p} 130 \mathrm{Cas}$ to $14-3-3$ protein is dramatically diminished when cells are detached from ECM, however, is rapidly recovered when cells are replated on fibronectin. Subcellular localization studies demonstrate co-localization of p130Cas and 14-3-3 protein at the leading edge of migrating cells. These data suggest that interaction of p130Cas and 14-3-3 protein may contribute to biological responses regulated by cell adhesion and migration [178]. 
1.2.2.3.2.4 The src-binding domain and binding proteins. The $\mathrm{p} 130 \mathrm{Cas}$ is a major Src substrate. In addition to the FAK-mediated recruitment of Src to p130Cas, the Src-binding domain (SBD) of p130Cas can directly bind to Src-family kinases (Scr, Fyn, Lck, Lyn and Hck) $[179,180]$. This domain contains two binding motifs: a proline-rich region (RPLPSPP) for Src SH3 domain binding, and a YDYV motif for Src SH2 domain binding [136]. The p130Cas SBD promotes SD tyrosine phosphorylation by recruiting Src-family kinases. In addition, it also has a function in cell migration and sustained FA disassembly [173]. Nephrocystin is a mouse ortholog to a human protein whose loss of function is involved in the cystic kidney disease familial juvenile nephronophthisis. Yeast two-hybrid screening has revealed the interaction between p130Cas and the SH3 domain of Nephrocystin. Immunostaining reveals that Nephrocystin and p130Cas co-localize at the site of cell-cell contact in polarized Madin-Darby canine kidney (MDCK) cells, indicating that these protein may be involved in the establishment of epithelial cell polarity [181]. Moreover, it has been reported that integrin-mediated adenovirus entry requires the association between p130Cas and PI3K. Further study reveals that the PPR of p130Cas is the binding site for PI3K p85 subunit, and deletion of the proline-rich domain abolishes interaction and inhibits virus entry. These data suggest the involvement of PI3K in the integrin signaling pathway [182].

\subsection{The Cas-family C-terminal homology domain and binding proteins.}

The p130Cas and its family members retain a conserved $\mathrm{C}$-terminal region, termed Casfamily C-terminal homology domain (CCHD). Donato et al. have demonstrated that venus-tagged p130Cas is a component of FAs throughout FA assembly, maturation and disassembly. Both p130Cas N-terminal SH3 domain and CCHD are required for the proper FA localization of p130Cas. Deletion of either of them results in impaired p130Cas FA targeting, and loss of both domains completely abolishes the p130Cas FA localization [145]. The CCHD bears structural similarity to the four-helical bundle of the FAT domain of FAK [183], which may account for the function of CCHD as an FA targeting domain. However, in the absence of the p130 SH3 domain, the CCHD alone is not sufficient for properly targeting p130Cas to FAs [145]. Therefore, both CCHD and the SH3 domain are necessary for full tyrosine phosphorylation of p130Cas and its role in promoting cell migration. Additional studies are needed to elucidate the mechanism responsible for the role of CCHD in recruiting p130Cas to FAs.

CCHD has various binding partners such as novel SH2-containing proteins (NSP) [184], Ajuba [185] or BMX/Tec family kinase [186]. NPS family members, NSP1, NSP2/AND-34/BCAR3 (BCAR3), and NSP3/SHEP1/CHAT (NSP3), have been shown to interact with the C-terminus of P130Cas [184, 187, 188]. It is reported that NPS1 associates with p130Cas in the presence of EGF and insulin, which may be involved in the JNK signaling pathway [187]. BCAR3 was originally identified as having GEF activity. For example, BCAR-p130Cas interaction activates small GTPase Rap1, leading to enhanced Src activity and cell migration [184]. BCAR3-p130Cas interaction is enhanced in estrogen receptor-negative breast cancer cells and contributes to the induction of cyclin D1 expression-linked tamoxifen resistance [146, 189-191]. Another NSF family member NSF3 also interacts with the C-terminus of p130Cas along actin 
filaments and ruffle membranes [188]. NSF3-p130Cas interaction activates Rap1 via p130Cas-Crk-G3G signaling pathway [192]. Moreover, this interaction is essential for proper olfactory development in neuronal cells [193].

In addition to NSP family members, a non-receptor tyrosine kinase, Bmx/Etk has been shown to interact with p130Cas. Bmx/Etk induces p130Cas phosphorylation and Crk recruitment, therefore, mediates cell adhesion and migration [186]. Ajuba, a zyxin family member, is another p130Cas CCHD binding protein. Cells from Ajuba null mice reveal deficient cell migration, lamellipodia production, and reduced phosphorylation in FAK, P130cAS, Crk and DOCK180. Overexpression of Rac1 in null cells is sufficient to rescue cell migration defects, indicating that Rac1 is the downstream target for Ajubap130Cas interaction [185].

\subsection{P130Cas functional roles}

p130Cas is an ubiquitously expressed adaptor protein and has been reported to play essential roles in development, cell migration, tumorigenesis and metastasis, and bacterial pathogenesis [136, 152]. Tyrosine phosphorylation of p130Cas SD, upon various stimuli including integrin engagement [143], growth factor [194], insulin [195], glucose [196] and antigens [197], is considered to be the major event of p130Cas signaling. The association of tyrosine phosphorylated SD and Crk is the central step of p130Cas signaling pathway [55].

1.2.2.3.3.1 Development. Loss of the single Drosophila Cas gene, Dcas, has little effect on embryonic development, however, it is related to the severity of developmental phenotypes due to mutations in integrin pathway. Fak56D and Dcas double knockout (corresponding to FAK and p130Cas) is embryonic lethal with defective cell polarity, mislocalization and reduced expression of E-cadherin. This study supports that Cas proteins have an important role in cell-cell adhesion signaling during development [198].

Genetic deletion of p130Cas gene in mice leads to embryonic lethality at E12.5, which cannot be compensated by other Cas-family members, indicating that p130Cas has a unique role in murine embryonic development [144]. p130Cas-deficient embryos exhibit a poorly developed heart and dilated blood vessels. Electron microscopic analysis reveals disorganized myofibrils and a disrupted Z-disk, preventing p130Cas-deficient embryos from efficient heart pumping; therefore gross morphological analysis demonstrated systemic congestion and overall retarded embryonic growth [144]. p130Cas has been implicated in heart and muscle development in different aspects. p130Cas, paxillin and FAK are localizing at the Z-lines of cardiomyocytes and required for the correct assembly of sarcomeres. Interruption of p130Cas impairs sarcomeric organization and results in cardiomyopathy $[199,200]$. p130Cas has also been identified as a negative regulator for the transcriptional factor Lim domain only 7 (Lmo7), which regulates genes involved in muscle and heart development [201]. In vitro, p130Cas deletion abolishes serum-induced myogenic differentiation by inhibiting F-actin assembly and disrupting 
nuclear translocation of serum response factor (SRF) co-activator MAL [202].

Furthermore, a $31 \mathrm{KD}$ caspase-cleavage product of $\mathrm{p} 130 \mathrm{Cas}$ direct interacts with myoblast determination protein 1 (MyoD1), and inhibits the expression of genes participating in myogenesis, suggesting that p130Cas $31 \mathrm{KD}$ form is a negative regulator for MyoD1-induced muscle differentiation [203].

p130Cas-deficient embryos also show small brains [144]. p130Cas is highly expressed in brain, especially in cerebellum during both embryonic and postnatal stages. p130Cas is extensively phosphorylated during cerebellar development and it is concentrated in the neuritis and growth cones of granule cells. Coimmunuoprecipitation demonstrated that p130Cas co-localizes with Src-family kinases, Crk, and cell adhesion molecules in granule cells. RNAi knocking-down of p130Cas inhibits the axon extension of granule cells, and overexpression with different p130Cas mutants shows SD is required for this process [204]. The p130Cas homolog in Drosophila, Dcas, is also required for correct axon guidance during development [205], indicating a conserved role of p130Cas in neuronal signaling.

Additionally, p130Cas has been demonstrated to play a role in retinal development. Conditional triple knockout of p130Cas, NEDD9 and Sin recapitulates the phenotype of loss of the $\beta 1$-integrin, including an overt disruption of ganglion cell layer (GCL) structure, and the formation of ectopic ganglion cell mass beyond the inner limiting membrane (ILM) [206]. Another study suggests that p130Cas, as the target of Src-family kinase Fyn, is required for oligodendrocyte process outgrowth, which is needed for myelination [207].

1.2.2.3.3.2 Cell motility. The best-elucidated cellular function of $\mathrm{p} 130 \mathrm{Cas}$ is regulating cell migration, which has been described in detail in previous sections. In summary, all p130Cas domains are necessary for efficient p130Cas signaling and cell migration. Both p130Cas N-terminal SH3 domain and CCHD are required for the proper FA localization and complete tyrosine phosphorylation of p130Cas [145]. The Src-family kinases recruited via the SBD are required for p130Cas SD tyrosine phosphorylation. Phosphorylated SD is capable of interacting with Crk and Nck [152]. Crk can form a complex with DOCK180 and ELMO to activate GTPase Rac by means of the GEF activity of DOCK180, therefore, promoting cell migration [113]. The binding of Nck to p130Cas SD facilitates signaling to ERK2, enhancing cell proliferation, cell cycle progression, and cell migration $[175,176]$. p130Cas has also been implicated in regulating FA disassembly, which is essential for efficient cell migration [173].

1.2.2.3.3.3 Cancer. Given the role of p130Cas in FA, cell migration, survival/apoptosis, and cell cycle, it is not surprising that aberrant expression and activation of p130Cas have been implicated to the progression and metastasis of several cancers [152]. Previous studies have demonstrated that cell detachment triggers rapid dephosphorylation of p130Cas accompanied by cell death, indicating the relevance of p130Cas in cell survival [208]. Pro-survival signals from ECM proceed through 
receptors, then through FAK and Src, to p130Cas. p130Cas can activate GTPase Ras and Rac, as well as JNK, ERK, and MAPK, to maintain viability [209]. Therefore, overexpression of p130Cas has been shown to promote cell survival in multiple studies $[146,208]$. Interestingly, multiple death stimuli can induce the generation of a $31 \mathrm{KD}$ p130Cas fragment. The $31 \mathrm{KD}$ fragment forms a heterodimer with transcription factor E2A, preventing E-box binding by E2A, and inhibiting E2A-mediated p21 transcription to induce cell cycle arrest [210]. p130Cas association with Crk activates JNK, and then leads to G1-S transition [211, 212]. Therefore, p130Cas can contribute to cell transformation by regulating cell cycle entry. Moreover, p130Cas associates with Zyxin family member Ajuba, thyroid hormone receptor interactor 6 (TRIP6), and Casinteracting zinc finger protein (CIZ) to induce matrix metalloproteinase (MMP) gene expression [160, 185, 213, 214].

The role of p130Cas (or BCAR1) in breast cancer progression has been intensively studied, as patients with highly expressed p130Cas are more likely to experience relapse and develop tamoxifen resistance [146, 215]. The p130Cas level in human breast tumors positively correlates with the level of receptor tyrosine-protein kinase erbB2 (ERBB2) [148]. And tumor samples from metastatic sites show higher p130Cas levels than in the primary tumors [216]. ERBB2 induces p130Cas and Crk association and enhances PI3K-AKT and ERK1/2 signaling downstream of ERBB2, leading to Rac1 activation and MMP9 secretion, therefore promoting cell migration and invasion [217, 218]. Silencing p130Cas in ERBB2-dependent breast caner impairs caner cell migration and invasion, and attenuates lung metastasis [217]. The BCAR3-p130Cas interaction has been proposed to confer tamoxifen resistance. BCAR3 was originally identified as having GEF activity that can activate small GTPase Rap1, leading to enhanced Src activity and cell migration [184]. BCAR3-p130Cas association is enhanced in estrogen receptor-negative breast cancer cells and contributes to the induction of cyclin D1 expression-linked tamoxifen resistance [146, 189-191].

p130Cas has been implicated to the progression of leukemias. BCR-ABL, involved in the leukemogenesis of chronic myelogenous leukemia (CML) and acute lymphoblastic leukemia (ALL), induces hyper-phosphorylation of p130Cas, drives the formation of p130Cas-Crk-C3G complex, activates GTPase Rap1, and promotes cell migration $[219,220]$. Together, these studies suggest that p130Cas expression and phosphorylation can be potential prognostic indicators, and p130Cas is a promising target for cancer treatment.

\subsection{Rationale and Hypothesis}

Previous studies have revealed that FAK can interact with Paxillin with its FAT domain. FAT is a protein-protein interaction domain, consisting of four alpha-helixes forming a helical bundle [30]. Based on the sequence analysis, comparison with the FAT domain and secondary structure prediction, we found that the Cas-family CCHD of p130Cas is a highly conserved region and may adopt a similar function to the FAT domain. Biological and biochemical evidences have demonstrated that both the N- 
terminal SH3 domain and C-terminal CCHD are necessary for the p130Cas FA localization, tyrosine phosphorylation and promoting cell migration [145]. However, the detailed information about how CCHD of p130Cas targeting FAs has not been elucidated yet. One possible binding partner is Paxillin, which mediates FAK targeting to FAs through interaction with the FAT domain of FAK [221]. Here we propose that p130Cas can interact with paxillin through its CCHD. 


\section{CHAPTER 2. METHODS}

\subsection{Nuclear Magnetic Resonance}

\subsubsection{Overview of NMR Spectroscopy}

Felix Bloch and Edward Purcell discovered nuclear magnetic resonance (NMR) back in 1946, and won the Nobel Prize in 1952, and then NMR spectroscopy was developed by Richard Ernst and Weston Anderson. NMR spectroscopy was originally used to study the chemical and physical properties of small- and macro-molecules for decades. In the early 1980s, Professor Kurt Wüthrich developed a method of sequential assignment, which is the foundation for studying the biological macromolecules by NMR spectroscopy. For his outstanding contribution in the development of NMR technique, Professor Kurt Wüthrich was honored with the Nobel Prize in chemistry in 2002. Since then, NMR spectroscopy has become a mature method widely used to study protein structure. NMR spectroscopy is a technique to monitor the absorption of energy associated with transitions of nuclei between adjacent nuclear magnetic energy levels. It determines the physical and chemical properties of the nuclei by relying on nuclear magnetic resonance. Furthermore, the surrounding chemical environment, such as chemical bonds and molecular conformations, influence the chemical properties of the nuclei. These subtle differences become the fingerprints of the nucleus in the molecule. NMR is very sensitive to detect these differences to reveal detailed information about the structure, conformation, dynamic, and chemical environment of molecules.

NMR spectroscopy and X-ray crystallography are two of the most important techniques used to study the three dimensional (3D) structures and dynamics of biological macromolecules at atomic level resolution. NMR shares some certain similarities with X-ray crystallography, as both methods determine the temporal and spatial averages of the molecular properties. For both structure determination techniques, measurements performed on an ensemble of molecules provide a description of the ensemble average, for example the type and relative population of conformational substates of the macromolecule. Beyond these similarities, there are several significant differences between these two important techniques. NMR spectroscopy determines the solution structure of a target protein; data represent an average over the dynamically oriented molecules in solution, whereas X-ray diffraction data represent an average over molecules arranged in a periodic crystal lattice. Base on the similarities and the differences, the applications of the two major structure determination techniques are different in practice. In general, X-ray crystallography can be used to solve very large protein complexes ( $>100 \mathrm{kDa}$ ), as long as crystals can be obtained; in contrast, solution NMR spectroscopy determines structures limited to smaller systems. However, the X-ray crystallography presents a static structure packed in the crystal lattice, which is like a snapshot of the moving object. The crystal packing, sometimes, can artificially affect the structures. On the other hand, NMR data representing averages in the nanosecond to second time range is capable of observing not only the structural information but also 
molecular dynamics and conformational equilibrium in solution. The size limitation of NMR structure determination is due to: first, the complexity of NMR data analysis, for example increasing size of the sample causes signal overlapping making assignment impossible; second, larger size slows down the tumbling rate that makes signal undetectable due to the faster decay.

\subsubsection{Basic NMR}

\subsubsection{Nuclear spin}

NMR is a physical phenomenon, describing the property and behavior of the nuclei in a presenting external magnetic field. The foundation of NMR technique is the quantum mechanical property of the nuclei, which is the spin. Nuclei are composed of protons and neutrons, and each of these components is considered as a spinning particle of spherical shape on their own axes. Spin is the intrinsic property of the particle and is not due to the rotation of the particle. The angular momentum $(\mathrm{J})$ is a vector and the direction of angular momentum is called the spin polarization axis. Spin comes in multiples of $1 / 2$ with plus or minus sign. In many atoms, such as ${ }^{12} \mathrm{C}$ and ${ }^{14} \mathrm{~N}$, these spins with plus or minus sign are paired against each other, therefore, the overall spin is counteracted ( $\operatorname{spin}=0$ ). However, in some atoms, such as ${ }^{1} \mathrm{H},{ }^{13} \mathrm{C}$, and ${ }^{15} \mathrm{~N}$, these nuclei possesses an overall net spin ( $\operatorname{spin}>0$ ). This unpaired net spin generates a magnetic dipole along the spin axis, which can be detected by NMR.

In the absence of an external magnetic field, each spin possesses its own micro magnetic field, which can be considered as a local magnetic moment vector. These randomly distributed micro magnets counteract their contributions to the total magnetic effect. The entire populations share the same energy level. But once presenting of an external magnetic field, the affected particles will rotate not only around the spin polarization axis, but also around the axis of this external magnetic field, which is called precession. Precession splits the particles of nuclei into two or more energy levels, which depend on the net spins (Figure 2-1). A quantum description of spin precessions is given by Equation 2-1. In this equation, the frequency of spin precession is termed as the Larmor frequency $(\omega 0)$, which is related to the gyromagnetic ratio of nuclei $(\gamma)$ and the strength of the external magnetic field (B0), shown in Equation 2-1.

$$
\omega_{0}=\gamma B_{0}
$$

Equation 2-1

The relationship between the magnetic moment $(\mu)$ and the angular momentum $(\mathrm{J})$ is given by Equation 2-2, which is related to the Plank's constant (h) and overall spin (I).

$$
\mu=\gamma J=\gamma \frac{h}{2 \pi} \sqrt{I(I+1)}
$$

Equation 2-2 


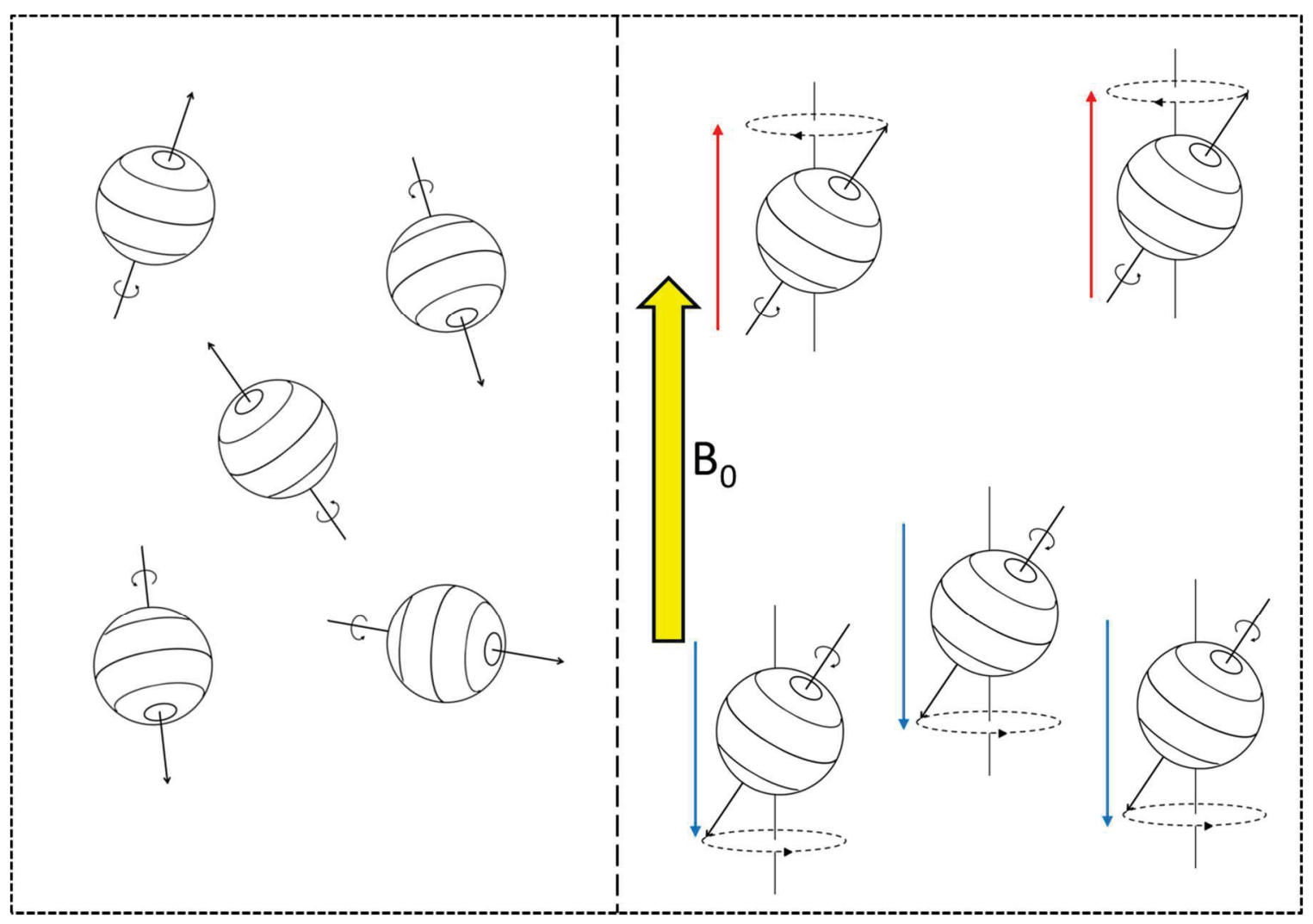

Figure 2-1. Nuclear spin precession and energy splitting in presenting external magnetic field. 
The gyromagnetic ratio of nucleus $(\gamma)$ is the basis of nuclear spin and each nucleus has its unique value, shown in Table 2-1.

\subsubsection{Transition energy and absorption of radiation}

The magnetic moment $(\mu)$ of spin is randomly orientated and sharing equal energy levels when in the absence of an external magnetic field; and by applying an external magnetic field, the magnetic moment can be split into two or more energy levels. The energy levels associated with the magnetic moment $(\mu)$ of spins are dependent on the strength of external magnetic field (B0) and the magnetic quantum number (m), as shown in Equation 2-3.

$$
E=-\mu \mathrm{B}_{0}=-m \gamma \frac{h}{2 \pi} \mathrm{B}_{0}
$$

\section{Equation 2-3}

The nuclei that can be used in protein NMR spectroscopy carry a total spin of $1 / 2$, such as $1 \mathrm{H}, 13 \mathrm{C}$ and $15 \mathrm{~N}$. Therefore the nuclear magnetic moment in the presence of external magnetic field can adopt two orientations with different energies corresponding to the magnetic quantum number, which are total spin of $1 / 2$ or $-1 / 2$ respectively. According to Boltzmann distribution, the lower energy level will contain slightly more nuclei than the higher level. In this case, the spins are allowed to jump from the lower energy level to the higher by absorbing the energy difference between two energy levels in the form of electromagnetic radiation. As described in Equation 2-4, the frequency of this electromagnetic radiation ( $v$ ), which is related to the energy difference, can be derived from Equation 2-3, where, $\Delta \mathrm{m}=1$ is the difference of magnetic quantum number between two energy levels.

$$
\begin{aligned}
& h v=\Delta E=\Delta m \gamma \frac{h}{2 \pi} B_{0} \\
& v=\frac{\gamma B_{0}}{2 \pi}
\end{aligned}
$$

The equation indicates the frequency of radiation required to excite a spin from the lower energy level to the higher one is identical to the Larmor frequency and is only related to gyromagnetic ratio $\gamma$ and B0. NMR probes the energy difference between two orientations of magnetic moment $\mu$ along B 0 and records the Larmor frequency of the nucleus.

\subsubsection{Pulsed Fourier transform NMR spectroscopy}

In pulsed Fourier transform-NMR (FT-NMR), a short rf pulse is applied to provide energy for exciting spins from a lower energy state to a higher energy state. The rf pulse contains many frequencies in a broad band and then excites all the resonances in a sample simultaneously. Due to thermodynamic balance, the excited spins will return to the lower energy state according to the Boltzmann distribution and emit the 
Table 2-1. Properties of nuclei important in protein NMR.

\begin{tabular}{lcccc}
\hline Nucleus & $\begin{array}{c}\boldsymbol{\gamma} \\
(\mathbf{1 0} \mathbf{~ r a d} / \mathbf{T s})\end{array}$ & $\begin{array}{c}\text { NMR } \\
\text { frequency at } \\
\mathbf{1 0 0}(\mathbf{M H z})\end{array}$ & Net spin & $\begin{array}{c}\text { Natural } \\
\text { abundance }\end{array}$ \\
\hline${ }^{1} \mathrm{H}$ & 26.752 & 100.0 & $1 / 2$ & 99.985 \\
${ }^{2} \mathrm{H}$ & 4.107 & 15.351 & 1 & 0.015 \\
${ }^{13} \mathrm{C}$ & 6.728 & 25.144 & $1 / 2$ & 1.108 \\
${ }^{14} \mathrm{~N}$ & 1.934 & 7.228 & 1 & 99.630 \\
${ }^{15} \mathrm{~N}$ & -2.7126 & 10.137 & $1 / 2$ & 0.37 \\
${ }^{19} \mathrm{~F}$ & 25.181 & 94.094 & $1 / 2$ & 100 \\
${ }^{31} \mathrm{P}$ & 10.8394 & 40.481 & $1 / 2$ & 100 \\
\hline
\end{tabular}


electromagnetic radiation. This signal will eventually decay away due to the action of relaxation in a time-dependent way; this signal is therefore called free induction decay (FID). The FID consists of a sum of decaying cosine waves whose frequencies represent the resonance frequencies of the nuclei in the sample. A mathematical operation called the Fourier Transformation is used to decompose the FID into its separate components and transform them from the time domain into the frequency domain which then gives a NMR spectrum.

\subsubsection{NMR Parameters}

\subsubsection{Chemical shift}

In an NMR spectrum, the nuclei are represented by their characteristic resonance frequencies. In the external magnetic field B0, electrons in an atom circulate around the direction of B0, and thus generate a small magnetic field at the nucleus; this small magnetic field thereby shields the nucleus slightly from B0. Since the electron density around each nucleus in a molecule varies according to the types of nuclei and chemical bonds, the Larmor frequencies of different nuclei vary depending on their own shielding behaviors based on the local chemical environment. Due to the interaction between the nucleus and surrounding electrons, the resonance frequencies of different nuclei of the same type also lie in a narrow frequency range instead of a single line. This effect is called chemical shift. The chemical shift is very sensitive to a multitude of structural, electronic, magnetic and dynamic variables and in principle contains a wealth of information on the state of the system under investigation. The chemical shift of a nucleus is described as the difference between the resonance frequency of the nucleus and that relative to the standard sample, shown in Equation 2-5.

$$
\delta=\frac{\left(v-v_{\mathrm{REF}}\right) \cdot 10^{6}}{v_{\mathrm{REF}}}
$$

Equation 2-5

Where $v$ is the resonance frequency of the nucleus; $v$ REF is the resonance frequency of the standard sample. This quantity is reported in ppm which is independent of the magnetic field strength B0. The standard for protons is often tetramethylsilane, $\mathrm{Si}\left(\mathrm{CH}_{3}\right)_{4}$, abbreviated as TMS, i.e. the chemical shift of the proton in TMS is defined as 0 ppm. The chemical shift is a very precise metric of the chemical environment around a nucleus.

\subsubsection{Spin-spin coupling}

A key feature exploited in NMR spectroscopy is the fact that the magnetic moment of the nucleus interacts with the small magnetic fields created by the spins of nearby nuclei. This spin-spin interaction can be used to correlate different nuclei with one another in a molecule. Spins interact with each other either 'directly', though-space, or 'indirectly', through-bond. The former are the basis for the nuclear Overhauser effect 
(NOE), which permits distance measurements between hydrogen nuclei; the latter are transmitted via polarization of bonding electrons and known as spin-spin coupling or $\mathrm{J}$ coupling.

Spin-spin coupling (J coupling) characterizes the trough-bond scalar interactions between two nuclei linked via covalent bonds in a chemical structure. The NMR signal will split due to the spin-spin coupling and the distance between two split absorption lines is called the $\mathrm{J}$ coupling constant $(\mathrm{J})$ or the spin-spin splitting constant; this constant is a measure of the magnetic interaction between two nuclei. $\mathrm{J}$ is field-independent and is customarily quoted in Hz. Normally, couplings over one (1J), two (2J, geminal coupling) and three bonds (3J, vicinal coupling) are observable. Vicinal coupling relies on the torsion angle formed by the four related nuclei and can be described by the famous Karplus equation, shown in Equation 2-6.

$$
{ }^{3} J=A \cos ^{2}(\theta)+B \cos (\theta)+C \quad \text { Equation 2-6 }
$$

Where $\theta$ is related to the torsion angle; $\mathrm{A}, \mathrm{B}$ and $\mathrm{C}$ are constants defined empirically.

\subsubsection{Nuclear overhauser effect (NOE)}

NMR-derived protein structure determination is mainly based on NOE measurements that demonstrate the proximity of protons in space and allow the determination of their approximate separation. Considering the simplest system with only two protons, called proton $\mathrm{A}$ and proton $\mathrm{B}$, each possesses a property known as magnetization; magnetization exchange between these two protons occurs by a process known as cross-relaxation. Because the cross-relaxation rates in both directions are equal, the magnetizations of two protons at equilibrium are equal. The cross-relaxation rate is proportional to two variables: $r-6$, where $r$ is the distance between proton $A$ and $B$, and tapp, the effective correlation time of the inter-proton vector. If the magnetization of one of the spins is perturbed, the magnetization of the second spin will change. The change in magnetization of proton $\mathrm{A}$ upon the magnetization perturbation of proton $\mathrm{B}$ is known as the nuclear Overhauser effect (NOE). The initial build-up rate of the NOE is equal to the cross-relaxation rate and, hence, proportional to $r-6$; therefore the NOE intensity falls off rapidly with increasing distance.

\subsection{Overview of NMR Hardware}

NMR spectrometers have now become very complicated instruments capable of performing an almost limitless number of sophisticated experiments. However, the really important parts of the spectrometer are not that complex to understand in outline. Broken down to its simplest form, the spectrometer consists of the following components: The intense, homogeneous and stable magnetic field; the probe which enables the coils that are used to excite and detect the signal to be placed close to the sample; high-power radio 
frequency (rf) transmitter capable of delivering short pulses; sensitive receiver to amplify the NMR signals; digitizer to convert NMR signals into a form which can be stored in computer memory; the pulse programmer to produce precisely pulses and delays; and the computer to control everything and to process data. Figure 2-2 displays a schematic representation of the major components of an NMR spectrometer and a few of the major interconnections. This overview briefly states the function of each component.

At the top of the schematic representation is the superconducting magnet of the NMR spectrometer that produces the B0 field necessary for NMR experiments. Immediately within the bore of the magnet are the shim coils for homogenizing the B0 field. Within the shim coils is the probe and the sample is positioned at the center of the probe. The probe contains rf coils and receiver coils: rf coils produce the $\mathrm{B} 1$ magnetic field which is necessary to rotate the spins by $90^{\circ}$ or $180^{\circ}$; receiver coils detect the signals emitted from the spins within the sample. Some probes also contain a set of gradient coils that produce a gradient in $\mathrm{B} 0$ along the $\mathrm{x}-, \mathrm{y}-$, or $\mathrm{z}$-axis; gradient coils are used for gradient-enhanced spectroscopy experiments.

The heart of the spectrometer is the computer. The operator of the spectrometer gives input to the computer through a console terminal with a mouse and keyboard. The computer controls all of the components of the spectrometer. The rf components under control of the computer are the rf frequency source and pulse programmer: the source produces a sine wave of the desired frequency; the pulse programmer sets the width, and in some cases the shape, of the rf pulses. The rf amplifier increases the pulses power from milli Watts to tens or hundreds of Watts. The computer of the sectrometer also controls the gradient pulse programmer which sets the shape and amplitude of gradient fields. The gradient amplifier increases the power of the gradient pulses to a level sufficient to drive gradient coils.

\subsection{NMR Spectroscopy}

\subsubsection{One-dimensional NMR Spectroscopy}

The simplest NMR experiment for FID detection is the one-dimensional (1D) experiment. The general simple 1D experiment in which a $90^{\circ} \mathrm{rf}$ pulse is introduced along the $\mathrm{x}$-axis and free induction decay (FID) signal is detected during $\mathrm{t} 1$.

1D NMR experiment consists of two sections: preparation and detection. During the preparation period, the spin systems are excited from their equilibrium states and flipped into the $x-y$ plane due to the application of an rf pulse. After the pulse, the excited spins precess around the z-axis and return to their equilibrium states by emitting

resonance signals which decays due to T2 relaxation (FID). The FID signal is detected by the receiver coils along the $\mathrm{x}$-axis. Fourier transformation is then used to transform the FID signal from time domain to frequency domain and thus gives a final NMR spectrum, which only contains a single resonance frequency. For a sample consisting of 


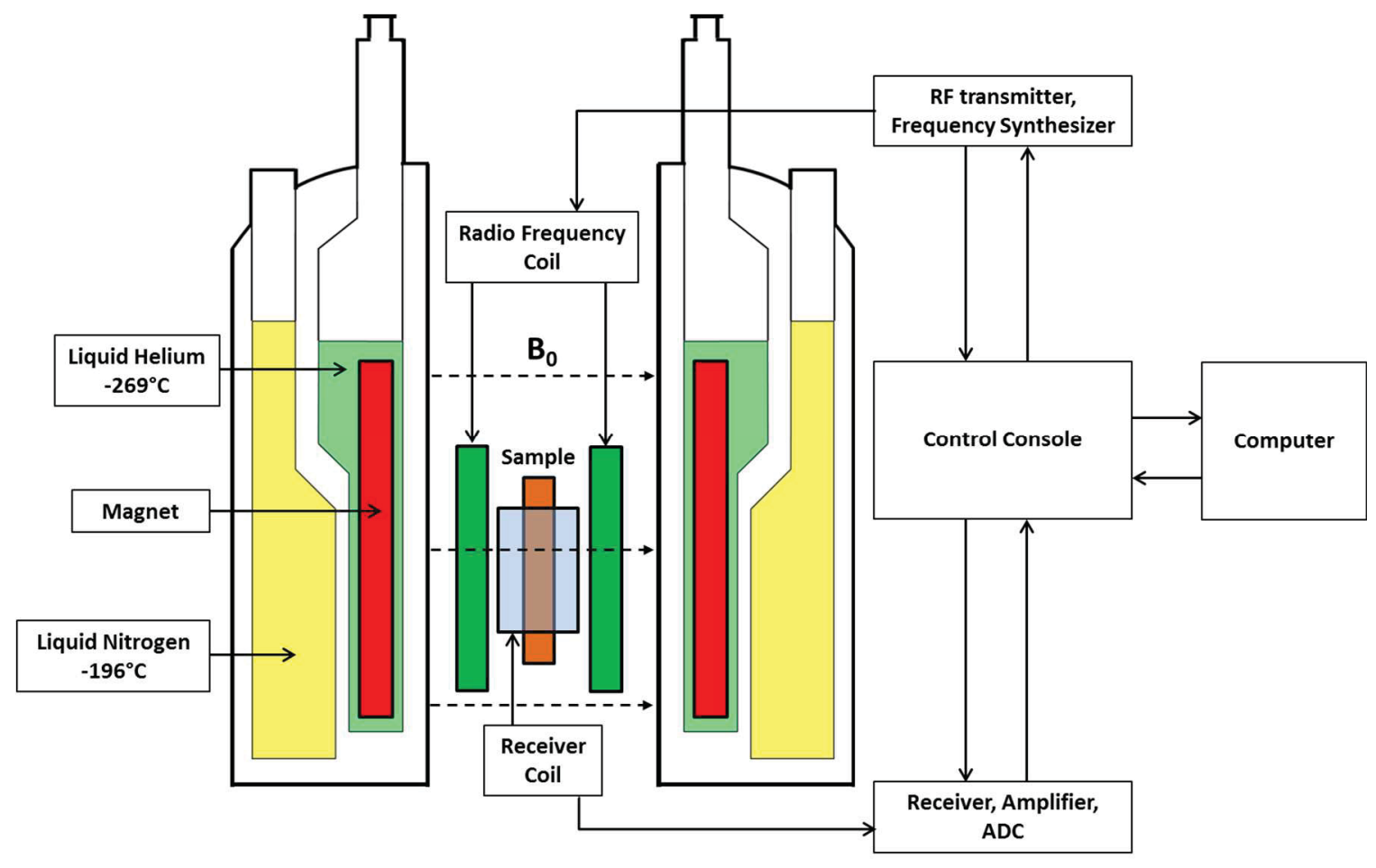

Figure 2-2. Overview of NMR spectroscopy hardware.

The major components of the NMR hardware are illustrated. 
biological macromolecules, the FID signal is composed of a sum of decaying cosine waves with different resonance frequencies coming from different nuclei in the sample; after Fourier transformation, therefore, the NMR spectrum contains variant resonance frequencies.

\subsubsection{Two-dimensional NMR Spectroscopy}

Theoretically, a 1D spectrum of the protein contains all the proton signals which can be used to determine protein structure. However, it is too complicated to interpret each peak as most of the signals overlap heavily. To overcome this problem, twodimensional (2D) NMR experiments are designed.

In 1D pulsed Fourier transform NMR, the signal is recorded as a function of one time variable resulting data is Fourier transformed twice to yield a spectrum which is a function of two and then Fourier transformed to give a spectrum which is a function of one frequency variable.

In 2D NMR, the signal is recorded as a function of two time variables, $\mathrm{t} 1$ and $\mathrm{t} 2$, and the frequency variables, usually called $\omega 1$ and $\omega 2$. Similar to 1D experiment, the construction of a 2D NMR experiment also contains preparation and detection periods. Other than these, a 2D experiment has an indirect evolution time t 1 and a mixing pulse. The whole process can be viewed as: (i) first, all the nuclei are excited by one or more pulses during the preparation period; (ii) the excited nuclei are allowed to precess freely during the evolution period t1; (iii) then, the nuclei are excited again by a mixing pulse which is used to transfer the magnetization from the first nucleus to the second one, thus setting up a connection between two nuclei; (iv) at last, FID signal is recorded as a function of the second time variable, $\mathrm{t} 2$, in the detection period. This sequence of events is called a 'pulse sequence' and the exact nature of the preparation and mixing periods determines the information found in the spectrum.

Notably, the signal is not recorded during time t1, but only recorded during time $\mathrm{t} 2$ at the end of the sequence. The 2D signal is recorded in the following way: First, $\mathrm{t} 1$ is set to zero; the pulse sequence is executed and the resulting FID is recorded during t2; then the nuclear spins are allowed to return to equilibrium. Second, t1 is then set to $\Delta 1$, the sampling interval in t1; the sequence is repeated and the second FID is recorded and stored separately from the first one; again the spins are allowed to equilibrate. Final, the whole process is repeated again for $t 1=2 \Delta 1,3 \Delta 1,4 \Delta 1$ and so on until sufficient data is recorded.

\subsubsection{The Types of 2D NMR Spectroscopy}

The cross-peaks visible in 2D NMR spectra correlate two spins together if they interact with one another during the mixing period. There are two mechanisms used for magnetization transfer during the mixing period, scalar coupling (J coupling) and dipolar 
coupling. NMR experiments that correlate different spins via scalar coupling are often referred to as correlation spectroscopy (COSY)-type experiments, which provide information on sequential connection of resonances; while the dipolar coupling mechanism transfer magnetization through spatially proximate dipole-dipole interactions, such as nuclear Overhauser effect spectroscopy (NOESY)-type experiments, which provide spatial correlation of resonances.

One the other hand, according to the resources of signals, 2D NMR experiments can be classified into two types, homonuclear and heteronuclear experiments. Experiments of homonuclear type produce signals coming from the same type of isotopes (usually ${ }^{1} \mathrm{H}-{ }^{1} \mathrm{H}$ ), such as 2D COSY and 2D NOESY; the signals of the heteronuclear type experiments result from the different types of isotopes (usually ${ }^{1} \mathrm{H}-{ }^{15} \mathrm{~N}$ or ${ }^{1} \mathrm{H}-{ }^{13} \mathrm{C}$ ), such as HSQC (heteronuclear single quantum correlation spectroscopy) and HMQC (heteronuclear multiple quantum correlation spectroscopy).

Below, the basic ideas of 2D NMR will be introduced by reference to the appearances of 2D COSY, 2D NOESY and HSQC spectra.

\subsubsection{2D COSY}

COSY-type experiments transfer magnetization between different nuclei via scalar coupling (chemical bonds). The cross-peaks in a 2D COSY spectrum represent signals arising from the interactions between protons connected by less than three chemical bonds in a protein sample. TOCSY (total correlation spectroscopy) is another type of correlation spectroscopy; the magnetization transfer in TOCSY-type experiments can disperse over the complete spin system via scalar coupling. Therefore the cross-peaks in a 2D TOCSY spectrum include the signals arising from the interactions between all of protons within one residue in a protein sample. So, COSY- and TOCSY-type spectroscopy can be used to group individual protons into one residue type in a protein sample.

\subsubsection{2D NOESY}

A 2D NOESY experiment is designed to establish the sequential connectivity of the spin systems and as well provide the spatial distance information of two spatially proximate spins. Magnetization transfer in NOESY experiment is via the dipolar coupling of spins due to their spatial proximity. In addition to the intra-residual peaks as seen in COSY and TOCSY spectra, the NOESY spectrum contains many inter-residual cross peaks arising from the spatial proximity of two protons in two residues which are probably not adjacent in the primary sequence of the protein. NOE signals in a NOESY spectrum are usually only visible if the distance between two protons is $<5 \AA$, and the peak magnitude is proportional to the sixth root of the distance between two interacting protons. 


\subsubsection{HSQC}

Other than the ${ }^{1} \mathrm{H},{ }^{13} \mathrm{C}$ and ${ }^{15} \mathrm{~N}$ are two basic elements for protein NMR. However, the natural abundances of ${ }^{15} \mathrm{~N}$ and ${ }^{13} \mathrm{C}$ isotopes are only $0.37 \%$ and $1.1 \%$ respectively. To increase the levels of ${ }^{15} \mathrm{~N}$ or ${ }^{13} \mathrm{C}$, recombinant protein can be grown in media that contain ${ }^{15} \mathrm{~N}$ or ${ }^{13} \mathrm{C}$ as the only nitrogen or carbon resources so that the ${ }^{15} \mathrm{~N}$ or ${ }^{13} \mathrm{C}$ content of the protein reaches $\sim 100 \%$.

The one-bond ${ }^{1} \mathrm{H}-{ }^{15} \mathrm{~N}$ or ${ }^{1} \mathrm{H}-{ }^{13} \mathrm{C}$ coupling is $\sim 95 \mathrm{~Hz}$ or $150 \mathrm{~Hz}$ respectively, which it is much larger than any of the long-range ${ }^{1} \mathrm{H}-{ }^{15} \mathrm{~N}$ or ${ }^{1} \mathrm{H}-{ }^{13} \mathrm{C}$ couplings. By utilizing this large difference of coupling, experiments can be devised to establish the correlation between ${ }^{15} \mathrm{~N} /{ }^{13} \mathrm{C}$ and the directly attached ${ }^{1} \mathrm{H}$; one of these types of experiments is socalled heteronuclear single quantum correlation spectroscopy (HSQC). In HSQC spectroscopy such as ${ }^{1} \mathrm{H}-{ }^{15} \mathrm{~N}$ HSQC or ${ }^{1} \mathrm{H}-{ }^{13} \mathrm{C}$ HSQC spectrum, the coordinate of a peak in one dimension is the chemical shift of ${ }^{1} \mathrm{H}$ and the coordinate in the other dimension is the chemical shift of ${ }^{15} \mathrm{~N}$ or ${ }^{13} \mathrm{C}$.

The ${ }^{1} \mathrm{H}-{ }^{15} \mathrm{~N}-\mathrm{HSQC}$ spectrum correlates the nitrogen atom of an $\mathrm{NH}$ group with its directly attached proton. Therefore, each peak in a ${ }^{1} \mathrm{H}-{ }^{15} \mathrm{~N}$ HSQC spectrum corresponds to an $\mathrm{NH}$ group in protein, and ${ }^{15} \mathrm{~N}$ chemical shift is usually plotted along y-axis and the ${ }^{1} \mathrm{H}$ chemical shift along $\mathrm{x}$-axis in the spectrum. Since each residue has only one backbone $\mathrm{NH}$ group, the spectrum can be considered as a fingerprint of the backbone conformation of the protein. The HSQC pulse sequence is often embedded in much more complex sequences which are used to record 2D and 3D spectra, such as ${ }^{15} \mathrm{~N}-\mathrm{HSQC}-\mathrm{NOESY}$ spectrum or ${ }^{13} \mathrm{C}-\mathrm{HSQC}-\mathrm{NOESY}$ spectrum.

\subsubsection{Multi-dimensional Spectroscopy}

There are two types of 3D NMR experiments. The first type basically consists of two $2 \mathrm{D}$ experiments. For instance, in a $3 \mathrm{D}{ }^{13} \mathrm{C}-\mathrm{HSQC}-\mathrm{NOESY}$ experiment, a 2D NOESY experiment is extended into the third dimension by adding a ${ }^{13} \mathrm{C}-\mathrm{HSQC}$ step after the mixing time of the 2D NOESY pulse sequence; data is then acquired after the HSQC step. The representatives of this type of $3 \mathrm{D}$ experiments are ${ }^{15} \mathrm{~N}-\mathrm{HSQC}-\mathrm{NOESY},{ }^{15} \mathrm{~N}-$ HSQC-TOCSY and ${ }^{13} \mathrm{C}-\mathrm{HSQC}-\mathrm{NOESY}$ and so on. Just like their corresponding 2D spectra, 3D TOCSY and 3D NOESY are usually used respectively to identify the individual spin systems and assign NOEs for future structure determination.

The second type of 3D NMR experiments is known as the triple-resonance experiments in which three different types of nuclei $\left({ }^{1} \mathrm{H},{ }^{13} \mathrm{C},{ }^{15} \mathrm{~N}\right)$ are correlated, such as so-called CBCA(CO)NH, HNCACB, HNCO and $\mathrm{HN}(\mathrm{CA}) \mathrm{CO}$ experiment and so on. One of the most important advantages of this type of $3 \mathrm{D}$ experiments is the simplicity of the spectrum because only a few signals exist on each frequency, thus leading to remarkable reduction of signal overlaps; another advantage of triple-resonance $3 \mathrm{D}$ experiments is their high sensitivity of $1 \mathrm{H}$ caused by the shorter magnetization transfer time and the 
smaller loss of magnetization. Triple-resonance 3D experiments are extremely powerful for assigning the chemical shifts for backbone atoms.

\subsubsection{NMR Solution Structure Calculation}

Protein structure determination using NMR spectroscopy is mainly based on the conformational information involved in NMR spectra. The geometric conformational constraints deduced form NMR spectra mainly include inter-proton distance constraints derived from NOE peaks in NOESY-type spectra and torsion angle constraints from coupling constants in COSY-type spectra. However, the NMR experimentally determined conformational constraints themselves are not sufficient to fully characterize a protein structure, as they are only based on a limited number of proton-proton distances. Therefore, the knowledge of empirical input data of a protein chemical structure, such as bond lengths, bond angles, torsion angles and van der waals atom radii and so on, is required. These kinds of empirical data together with NMR experimental constraints enable a reasonably exact structure determination.

There are several computer programs available for the calculation of protein $3 \mathrm{D}$ structures based on NMR constraints. To generate a 3D structure, a randomly folded starting structure is first calculated from the empirical data and the known amino acid sequence; the computer program then tries to fold the starting structure in such a way that the experimentally determined inter-proton distances and torsion angle constraints are satisfied by the calculated structures. In order to achieve this, the main computational methods comprise as common features a conformational search to locate the global minimum of a target function.

The target function value is the sum of all the differences between the actual distances and the input experimental constraints. The minimization of a target function can be achieved through molecular dynamics methods. Simulated annealing (SA) is a powerful molecular dynamics method, which takes place directly in the Cartesian coordinate system. In this method, a starting structure is heated to a high temperature in a simulation to allow the atoms to get a high thermal mobility, and then cooled down slowly in order not to get trapped in local energy minima. During many discrete cooling steps the starting structure can evolve towards the energetically favorable final structure under the influence of a force field derived from the constraints. SA is very efficient at locating the global minimum of the target function. Finally, a good ensemble of structures with the lowest target function values is reported as a representation for the real structure. The quality of a structure is assessed by the root-mean-square deviation (RMSD) between the atoms of the individual conformers in the ensemble.

So far, programs available for NMR protein structure determination employ in principle two different approaches: one is distance geometry combined with simulated annealing (DGSA), which is based on a calculation of distance matrices for each pair of atoms derived from all available distance constraints, bond lengths, bond angles, torsion angles as well as van der Waals radii; the other is torsion angle dynamics with simulated 
annealing (TAD), which performs molecular dynamics simulations by using the torsion angles as the only degree of freedom and the upper and lower distance limits as main constraints during calculations. 


\section{CHAPTER 3. STUDY OF HELICAL CONSTRAINED PEPTIDE}

\subsection{Background}

FAK is a nonreceptor kinase consisting of a central catalytic domain flanked by large $\mathrm{N}$ - and $\mathrm{C}$-terminal noncatalytic domains. The $\mathrm{C}$-terminal region is rich in proteinprotein interaction sites. This portion of FAK, called FAK-related nonkinase (FRNK)[59], when autonomously expressed acts as a negative regulator of FAK activity [222] and blocks the formation of focal adhesions [223]. FRNK consists of several proline-rich regions that act as binding sites for many $\mathrm{SH}$-containing proteins followed by a C-terminal focal adhesion targeting (FAT) domain[59]. The FAT domain targets sites of focal adhesion in the cell [224, 225] and microinjections of this domain in cells exhibited decreased cell motility[226]. The FAT domain $(\sim 15.5 \mathrm{kDa})$ is composed of four helices that form a right turn elongated bundle that is maintained by hydrophobic interactions $[30,121,183]$ and is also the binding site for the focal adhesion protein paxillin which is one of the early binding partners for FAK in focal adhesion complexes $[72,121,221,227,228]$.

Paxillin is a multi-domain adaptor protein that localizes in cultured cells primarily to sites of cell adhesion to the extracellular matrix (ECM) and is a major target for tyrosine kinases during various cellular events associated with cell adhesion and growth control [56]. It is highly conserved between species, having 90\% identity between chicken and humans $[80,81]$. Paxillin contains many protein-binding modules that allow it to bind a variety of structural and signaling molecules. The $\mathrm{C}$-terminal domain consists of 4 LIM (double zinc finger) motifs [72]. The N-terminal domain consists of 5 protein binding LD motifs with the consensus sequence LDXLLXXL, termed LD1 through LD5 [82]. It has been proposed that paxillin binds to FAT through the interaction of two of these LD motifs, LD2 and LD4, via two hydrophobic patches on opposite faces of the 4 helix bundle of FAT, and the structure of LD2/LD4 complex with the FAK FAT domain has been solved by our lab.

FAK is localized to focal adhesions via its C-terminal FAT domain through association with paxillin, a multi-domain adaptor protein also associated with cell adhesion and growth control. The FAT surface has two hydrophobic patches on opposite faces of the four helix bundle as protein binding sites; helices $\mathrm{H} 1$ and $\mathrm{H} 4$ form one site and helices $\mathrm{H} 2$ and $\mathrm{H} 3$ form another. Paxillin binds the FAT domain through two of its N-terminal helical LD motifs, termed LD2 and LD4; the LD2 motif binds to the H1/H4 site and the LD4 motif binds to the $\mathrm{H} 2 / \mathrm{H} 3$ site. Our previous study showed that while LD2 has the ability to bind either the $\mathrm{H} 1 / \mathrm{H} 4$ or the $\mathrm{H} 2 / \mathrm{H} 3$ site, LD4 binds specifically to the $\mathrm{H} 2 / \mathrm{H} 3$ site. The binding affinity of LD2 and LD4 individually to the FAT domain is relatively weak, thus, the binding of both LD motifs is required to form a stable FAKpaxillin complex. Therefore, the FAK-paxillin complex has two potential sites that could be targeted to inhibit complex formation. Furthermore, it has been reported that phosphorylation of Ser 273 in the LD4 motif of paxillin plays an important role in the focal adhesion turnover mechanism, resulting in a significant decrease in binding affinity 
to FAK and an increased association between paxillin and Git1. Expression of paxillin lacking the LD4 motif in pxl-/- cells results in an 11-fold decrease in the rate of focal adhesion disassembly.[13] It has also been shown that the integrity of the $\mathrm{H} 2 / \mathrm{H} 3$ site on FAT is crucial for maximal binding of paxillin, whereas only the $\mathrm{H} 1 / \mathrm{H} 4$ site is required for efficient localization of FAK to FAs.[14] Together, these studies suggest that LD2 and LD4 binding to FAK are not redundant, and play differential roles in the mechanisms of focal adhesion localization, focal adhesion kinase activation and focal adhesion turnover.

In previous work our lab showed that LD4 is mostly unstructured in solution and folds into an alpha-helical conformation upon binding to FAT and that the binding affinity of LD4 was proportional to its helical propensity. $[10,11]$ In this study we use a helical constrained approach to force the LD4 peptide to maintain a helical structure for enhancing the binding affinity to FAT, in order to block paxillin/FAK interaction.

\subsection{Experiment Procedures}

\subsubsection{Protein Expression and Purification}

In order to create a model for studying the interaction between LD4 and FAT, we generated a construct called FAT-LD2 that covalently linked the LD2 motif of paxillin onto FAT. The cDNA encoding the FAT domain (residues 916 to 1053) was subcloned into a pET28a vector. The N-terminal His-tagged FAT domain was subsequently expressed in E. coli strain BL21(DE3)pLysS. FAT-LD2 was sub-cloned into pET28a vectors where LD2 oligos were inserted in a BamHI site downstream of FAT.

Oligonucleotides that contained BamHI restriction sites and the LD2 sequences flanked 5 ' by glycine serine repeats, $(\mathrm{GGS})_{2} \mathrm{GS}(\mathrm{GGS})_{2}$ were synthesized at the Hartwell Center for Bioinformatics and Biotechnology at St. Jude Children's Research Hospital.

Incorporation of FAT-LD2 into pET28a was verified by sequencing using the T7 terminator primer. Expression of FAT-LD2 was initiated through inoculating $100 \mathrm{ml}$ of LB media containing $30 \mu \mathrm{g} / \mathrm{ml}$ Kanamycin and $34 \mu \mathrm{g} / \mathrm{ml}$ Chloramphenicol. $10 \mathrm{ml}$ of the starter culture was used to inoculate each of the 6 liter aliquots of LB media containing the final concentration of $30 \mu \mathrm{g} / \mathrm{ml}$ Kanamycin and $34 \mu \mathrm{g} / \mathrm{ml}$ Chloramphenicol. The cultures grew at $37^{\circ} \mathrm{C}$ in the shaker at $250 \mathrm{rpm}$ for four to five hours to reach an $\mathrm{OD}_{600}$ of 0.6 to 0.8 . Induction of T7 RNA polymerase was performed by addition of IPTG at a final concentration of $1 \mathrm{mM}$. Then the cultures were incubated at $37^{\circ} \mathrm{C}$ for another five hours at $250 \mathrm{rpm}$. Cell was lysed in the buffer of $50 \mathrm{mM}$ potassium phosphate (KPi) with $\mathrm{pH}$ at $6.5,500 \mathrm{mM}$ sodium chloride $(\mathrm{NaCl})$, and $1 \%$ Triton $\mathrm{X}-100$, by sonication. FATLD2 then was purified by a general protein purification strategy, which included Ni-Co affinity chromatography, removing the His-tag by thrombin cleavage, and final purifying by size exclusion chromatography. E.coli cell lysates from 6 liters of cultures were loaded on a gravity column, which packed $6 \mathrm{ml}$ of Ni-Co sepharose at the flow rate of approximately $1 \mathrm{ml}$ per minute. The loaded column was washed by 10 -column volumes

of lysis buffer, and then the target protein was eluted with $50 \mathrm{mM} \mathrm{KPi}$ (pH6.5), $300 \mathrm{mM}$ 
$\mathrm{NaCl}$, and $250 \mathrm{mM}$ imidazole. Thrombin was added to FAT-LD2 overnight at $4{ }^{\circ} \mathrm{C}$. FAT-LD2 and His-tag mixtures were dialyzed against $20 \mathrm{mM} \mathrm{KPi}$ (pH6.5) with $1 \mathrm{mM}$ sodium azide. Then FAT-LD2 was concentrated and further purified with gel filtration chromatography by using the AKTA FPLC system. Purified FAT-LD2 was aliquoted into $500 \mu \mathrm{l}$ fractions of approximate $0.2 \mathrm{mM}$ each, that froze at $-80^{\circ} \mathrm{C}$. Isotope labeled protein was prepared using morpholinepropanesulfonic acid-buffered medium containing ${ }^{15} \mathrm{NH} 4 \mathrm{Cl}(1 \mathrm{~g} /$ liter $)$ and ${ }^{13} \mathrm{C} 6$-glucose $(2.5 \mathrm{~g} /$ liter $)$, and then purified by the same protocol.

\subsubsection{Stapled Peptides Synthesis}

Two types of helically constrained LD4 peptides have been synthesized in this study, one is using a lactam bridge approach and the other is cross-linked by using hydrocarbon stapling. For the fluorescent polarization study, the FAM-labeled peptides have been produced. All peptides were made by Hartwell Center at St. Jude Children's Research Hospital on a Liberty microwave peptide synthesizer (CEM Corporation). Standard solid-phase Fmoc peptide chemistry was used for peptide synthesis. As an example, lactam bridged LD4 peptide was synthesized in following steps:

\subsubsection{Peptides synthesis}

a. Coupling was done using Fmoc-amino acids (5eq), HBTU (5eq), and DIEA (10eq) in anhydrous DMF under microwave conditions for $5 \mathrm{~min}$ at $75^{\circ} \mathrm{C}$. Double coupling was performed on every amino acid.

b. For lactam peptides, Boc-protected Serine was used as the last residue.

c. For FAM-labeled peptides, Fmoc-aminobutyric acid was used as the last residue.

\subsubsection{On-resin macrolactam formation}

a. Deprotection of the $\operatorname{Pd}(0)$ labile protecting groups, which were Fmoc Lys(Alloc)-OH, and Fmoc Glu(allyl)-OH, was accomplished by treatment with Tetrakis (triphenylphosphine palladium (0)) (0.5eq), triphenylphosphine (2eq), and 1,3-dimethylbarbituric acid (5eq) in DCM:THF (1:1) under microwave conditions for $15 \mathrm{~min}$ at $65^{\circ} \mathrm{C}$. This was followed by extensive washing with DCM and a $0.5 \%$ solution of diethyldithiocarbamic acid sodium salt $(w / v)$ in DMF.

b. Cyclization was carried out using TFFH (0.5eq), PyAOP (3eq), HOAt (3.2eq), and DIEA (6eq) with $15 \%$ TFE in DMF for $15 \mathrm{~min}$ at $70^{\circ} \mathrm{C}$ under microwave conditions.

c. Following the cyclization the lysine of the remaining uncyclized peptide was acetylated using $\mathrm{Ac}_{2} \mathrm{O}$ and DIEA for $2 \mathrm{~min}$ at $65^{\circ} \mathrm{C}$ under microwave conditions, to minimize side products. 


\subsubsection{Fluorescent labeling}

a. For FAM-labeled peptides, after the macro-lactam formation, the peptides were treated with $20 \%$ piperidine to remove the Fmoc group on the terminal animobutyric acid moiety.

b. The resin bound peptides were reacted with HATU activated FAM in DMF for $15 \mathrm{~min}$ at $70^{\circ} \mathrm{C}$ under microwave conditions.

\subsubsection{Peptide cleavage and purification}

a. Peptides were cleaved from the resin with concomitant side-chain deprotection using $82.5 \%$ TFA, $5 \%$ water, $5 \%$ thioanisole, $2.5 \%$ EDT, $2.5 \%$ triisopropylsilane and $2.5 \%$ Phenol for $2 \mathrm{hrs}$ at room temperature.

b. The peptides solutions were filtered and precipitated by adding cold diethyl ether.

c. After centrifugation, the peptides were brought up in water and lyophilized.

d. The lyophilized peptides were HPLC purified using a Waters Alliance HPLC

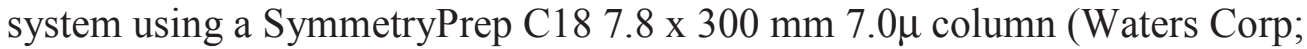
Milford, MA).

e. Analytical HPLC and Mass spectra were run on the purified peptides.

The hydrocarbon-stapled peptides were synthesized similarly by Fmoc solid phase synthesis method using Rink amide MBHA resin. For normal amino acides, couplings were performed as shown above. Instead of natural Alanine, the (s)-Fmoc- $\alpha-$ (2'-pentenyl)-alanine, which containing a long hydrocarbon side chain, has been use to form the linker.

\subsubsection{Circular Dichroism Studies}

Spectra were obtained with an Aviv62DS CD spectrometer (Aviv, Lakewood, N.J.) and processed using Origin (OriginLab Corporation). All experiments were performed at room temperature $\left(25^{\circ} \mathrm{C}\right)$ using a quartz cuvette with a $0.1 \mathrm{~cm}$ path length. The parameters used for the measurements were as follows: $0.5 \mathrm{~nm}$ step resolution, 10 second average signaling time, and $1 \mathrm{~nm}$ bandwidth. Concentration of samples ranged from 20 to $40 \mu \mathrm{M}$ in $20 \mathrm{mM}$ phosphate buffer with a $\mathrm{pH}$ at 6.5 .

\subsubsection{Isothermal Titration Calorimetry Studies}

ITC experiments were performed using a Microcal ITC200 instrument (Microcal). All the synthetic helical constrained LD4 peptides were dissolved in $20 \mathrm{mM} \mathrm{KPi}$ buffer (pH6.5), in a $2 \mathrm{mM}$ stock, and then adjusted $\mathrm{pH}$ to 6.5 before use. Purified FAT-LD2 protein was dialyzed against the same buffer used for dissolving peptides. The sample cell of the calorimeter was loaded with FAT-LD2 of a concentration of $0.1 \mathrm{mM}$. The 
syringe was loaded with LD peptides of a final concentration of $1 \mathrm{mM}$ for titrations. All solutions were degassed by high speed centrifuging for 10 minutes. Titrations were performed at $25^{\circ} \mathrm{C}$ with injection volumes of $2 \mu \mathrm{l}$ of a total 19 injections, and a spacing of 120 seconds in between each injection. The data were fit using a one-site binding model in the Origin ITC data analysis software (v. 7.0).

\subsubsection{Fluorescent Polarization}

Fluorescence polarization measurements were taken using a Fluorolog fluorimeter (Jobin Yvon, Horriba) equipped with polarizers. A $1 \mathrm{~cm}$ cuvette containing $1.5 \mathrm{ml}$ of a 0.5 $\mu \mathrm{M}$ solution of fluorescently labeled LD4 peptides were titrated with successive $0.5-20$ $\mu \mathrm{l}$ aliquots of $0.5 \mathrm{mM}$ FAT-LD2. The experiments were done at $\mathrm{pH} 6.5$ in $10 \mathrm{mM}$ phosphate buffer. Anisotropy was measured at an emission wavelength of $513 \mathrm{~nm} 3 \mathrm{~min}$ after each addition to allow the system to come to equilibrium. Change in anisotropy for binding was plotted and fitted to a standard one-site binding model in order to determine the binding constant $\mathrm{Kd}$ and the maximum change in anisotropy, Bmax, using Prism (version 4.0 for Windows, GraphPad Software)

The competitive binding assay and inhibition assay using fluorescent polarization measurement have been performed by PHERAstar FS plate reader (BMG LABTECH GMBH). In both assay, the 384 wells plate was used for sample preparation. For competitive binding assay, $0.5 \mu \mathrm{M}$ fluorescently labeled LD4 peptide as the probe mixed with $20 \mu \mathrm{M}$ competitor peptides added into each testing wells, then titrate FAT-LD2 protein with a concentration from 0 to $100 \mu \mathrm{M}$. For the inhibition assay, $0.5 \mu \mathrm{M}$ fluorescently labeled LD4 peptide was mixed with FAT-LD2 of a fixed concentration of $20 \mu \mathrm{M}$ or $25 \mu \mathrm{M}$ with 10 minutes incubation to achieve FAT-LD2/LD4 complex. Then unlabeled helical constrained peptides have been titrated into protein sample mixture as the inhibitors with a concentration from $0 \mu \mathrm{M}$ to $100 \mu \mathrm{M}$. Final data was plotted and processed by software Origin.

\subsubsection{NMR Spectroscopy and Chemical Shift Perturbation}

NMR data for signal assignment were acquired with Bruker $600 \mathrm{MHz}$ and Bruker Avance $800 \mathrm{MHz}$ (with cryoprobe) spectrometers at $25^{\circ} \mathrm{C}$. Sample concentrations for nuclear magnetic resonance experiments were typically 0.2 to $0.5 \mathrm{mM}$ in $20 \mathrm{mM}$ potassium phosphate buffer, $\mathrm{pH} 6.5,10 \% \mathrm{D} 2 \mathrm{O}$, and $0.1 \%$ sodium azide. Data were processed using the program Topspin 3.1 and displayed by CARA. Proton chemical shifts were referenced to $\mathrm{H}_{2} \mathrm{O}$ peaks $(4.75 \mathrm{ppm}) .15 \mathrm{~N}$ chemical shifts were referenced indirectly to DSS using absolute frequency ratios. The program SPARKY (T. D. Goddard and D. G. Kneller, SPARKY 3, University of California, San Francisco) was used for data analysis and assignment.

NMR data for binding studies were acquired on a Bruker $600 \mathrm{MHz}$ spectrometer at $25^{\circ} \mathrm{C}$, and processed as described above. $15 \mathrm{~N}$ and $1 \mathrm{H}$ chemical shift values for the 
displaced peaks in the ${ }^{15} \mathrm{~N}-\mathrm{HSQC}$ titration experiments were determined for each of the successive titration points using SPARKY. The average chemical shift perturbation was calculated as using the following Equation 3-1[229]:

$$
\Delta \delta_{\text {avg }}=\sqrt{\frac{\left(\frac{\Delta \delta N}{5}\right)^{2}+\Delta \delta H^{2}}{2}}
$$

\section{Equation 3-1}

In this equation $\Delta \delta \mathrm{N}$ is chemical shift change of the amide nitrogen and $\Delta \delta \mathrm{H}$ is the chemical change of the amide proton. The amide nitrogen chemical shift change is divided by 5 to account for the scale difference of the nitrogen and proton dimensions.

\subsection{Results}

\subsubsection{Single Binding Site FAT for Studying Helical Constrained LD4 Peptides}

In a previous study from our lab we generated the FAT construct for studying the structure of paxillin complex with FAT. Our study showed the FAT domain has two binding sites, which are composed of helices $\mathrm{H} 1$ and $\mathrm{H} 4$, and helices $\mathrm{H} 2$ and $\mathrm{H} 3$ on the opposite site of a four-helix bundle. The motifs LD2 and LD4 from paxillin bind to FAT on the $\mathrm{H} 1 / \mathrm{H} 4$ site and the $\mathrm{H} 2 / \mathrm{H} 3$ site respectively. In order to perform the experiment to test the binding of constrained LD4 peptides to FAT and identify potential small molecular inhibitors we had to design a new construct to build our model. We proposed to use a single binding site opened FAT protein, and chose the LD4 peptide to modify as inhibitors to achieve our goal.

There are three major reasons that we need to create a single-site FAT model, as well as choosing the LD4 motif that can better serve our purpose. First, the binding affinity of individual LD motifs is relatively weak, making it impossible for paxillin to form a complex with FAT through only a single LD motif. Hence, disturbing one interaction of the LD motifs with FAT can efficiently affect the FAT/paxillin complex formation. Second, binding of both LD2 and LD4 motifs is required to achieve maximal association of paxillin to FAT. Therefore, a single LD motif with a two-site FAT will be hard to study the binding. Third, LD2 can bind to both sites of FAT while LD4 specifically targets $\mathrm{H} 2 / \mathrm{H} 3$ site. Also previous studies from our lab suggest LD4 motifs form a coil-like structure in solution that leads to a prefect model for investigating the enhancement of binding by improved helicity through the helical constraining technic.

Based on these reasons, we designed an $\mathrm{H} 1 / \mathrm{H} 4$ site closed and $\mathrm{H} 2 / \mathrm{H} 3$ site opened single site FAT construct, which is the FAT protein linked with the LD2 motif of paxillin. We covalently attached the individual LD2 motif to the C-terminal end of the FAT domain through (GGS) linkers, which allows the LD2 motif occupying the H1/H4 site with the H2/H3 site open, shown in Figure 3-1. Then the study will focus on the helical constrained LD4 peptide targeting only the $\mathrm{H} 2 / \mathrm{H} 3$ site. 


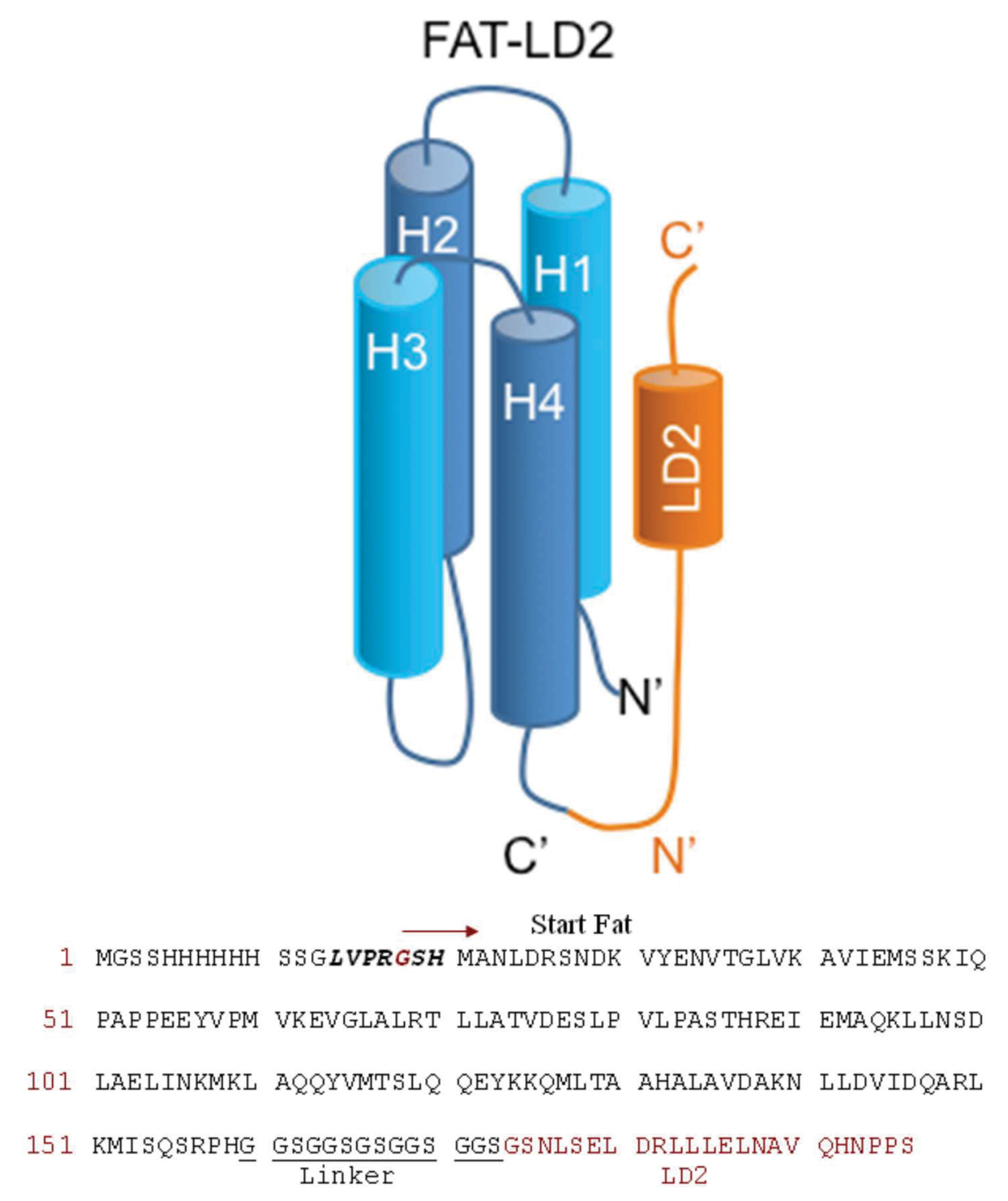

Figure 3-1. Single binding site construct FAT-LD2.

The single binding site model is illustrated in the figure and the sequence. The LD2 motif of paxillin has been covalently linked to the C-terminal of FAT domain by a (GGS)x linker. 


\subsubsection{Comparison of Structure of FAT-LD2 and FAT/LD2 Complex}

The FAT-LD2 construct was subcloned into BL21(DE3)pLysS E.coli stain and the protein has been expressed and purified by the standard protocol. Our lab solved the solution structure of FAT-LD2. Since the FAT-LD2 has been modified as a single-site FAT protein, we compared our solution structure of FAT covalently linked with the LD2 motif with the crystal structure of the natural FAT domain complex with LD2, by superimposing the H1-H4 binding pockets of the FAT-LD2 construct (2L6G) and the Xray structure of FAT bound by LD2 (1OW7), to verify our structure model. The peptides used in the X-ray study are shorter by several residues than ours used in the solution structures. Comparison of the LD2 motif shows it to be shifted by half a helix turn towards the H1-H4 loop in the solution structure. The ELD motif in the sequence of LD peptides was suggested to be involved in charge pairing interactions that helped to define the positioning and orientation of LD peptides within their binding pockets. However adequate electron density was not observed to confirm this theory. A closer look at these interactions in the solution structure of the FAT-LD2 construct reveal that residues Glu 139 and Asp 141 of the LD2 motif are indeed close enough to form a salt bridge with residues Lys 934 and Lys 1033 of the FAT domain respectively. In the x-ray structure, only the Asp 141 to Lys 1033 salt bridge is supported. Overall, identical to the crystal natural FAT/LD2 complex structure our FAT-LD2 fusion protein shows well-folded FAT domain binding with the LD2 motif. The comparison suggests that testing of helical constrained LD4 peptide on our FAT-LD2 will achieve the same result as on the FAT/LD2 complex.

\subsubsection{Design of Helical Constrained LD4 Peptide}

Studies showed that the LD4 peptide is mostly an unstructured coil-like fragment in solution and folds into an alpha-helical conformation upon binding to the $\mathrm{H} 2 / \mathrm{H} 3 \mathrm{site}$ FAT domain. Therefore, entropy is an important factor in the binding, and increasing the helical propensity of LD4 should significantly enhance the peptide's binding affinity to the FAT domain. Many strategies have been developed to increase peptide helicity by constraining conformation, and perhaps the most effective approach is to link peptide side chains at the $i$ and the $i+4$ position, shown in Figure 3-2. In this study, we mainly used the macro-lactam approach and hydrocarbon stapling to constrain the helical conformation of the LD4 peptides.

When bound to the FAT domain, LD4 forms an amphipathic alpha-helix with its hydrophobic face oriented towards the hydrophobic patch formed between helices $\mathrm{H} 2$ and H3 of FAT. Residues Leu 6, Leu 9 and Leu 11 of LD4 contribute the major hydrophobic interaction for LD4 associating with the hydrophobic patch on $\mathrm{H} 2 / \mathrm{H} 3$ site of FAT. Charge residues Glu 5, Asp 7 and Ser 12 of LD4 forming salt bridges with FAT provide anchor points to position LD4 specifically on H2/H3, shown in Figure 3-3. Therefore, in principle, the linker needs to be positioned in the center of the helix while not disturbing the hydrophobic binding surface. Based on these features, we designed two groups of constrained LD4 peptides with the linker on different positions, which are 


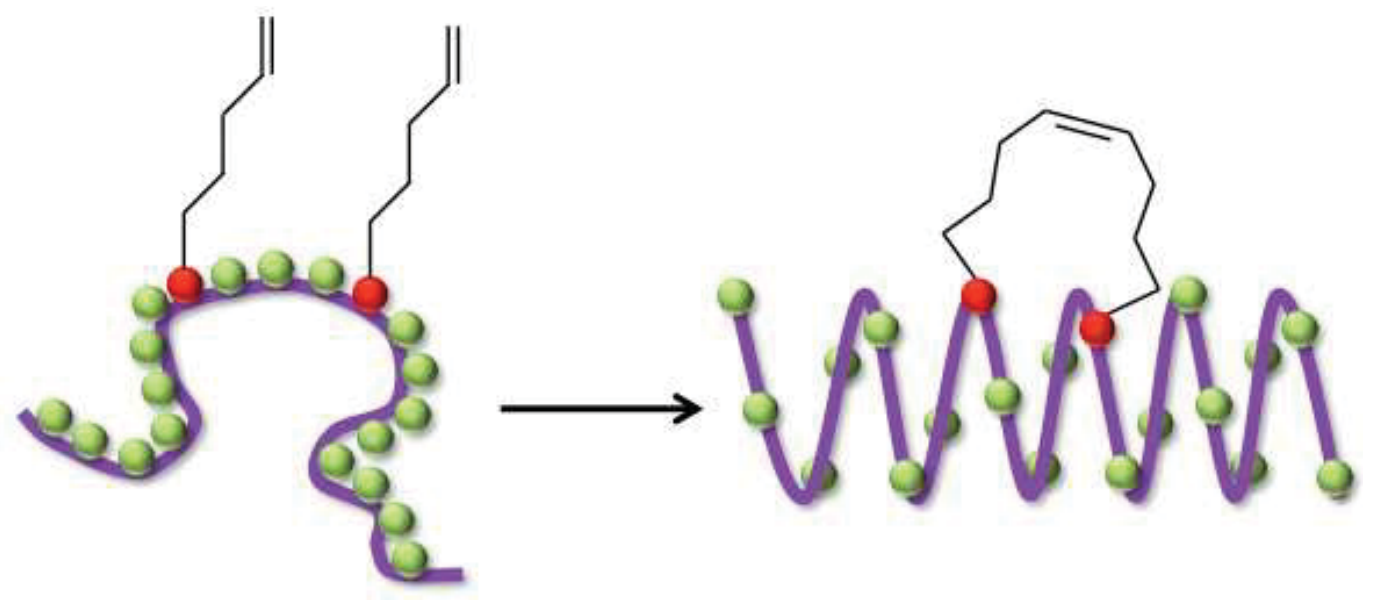

Figure 3-2. Hydrocabon stapling for constraining the helical structure of LD4 peptides.

The selected residues on $\mathrm{i}$ and its $\mathrm{i}+4$ positions have been cross-linked by the side chains of these residues. By constraining the structure that forces the peptide to form and maintain a helical conformation. 

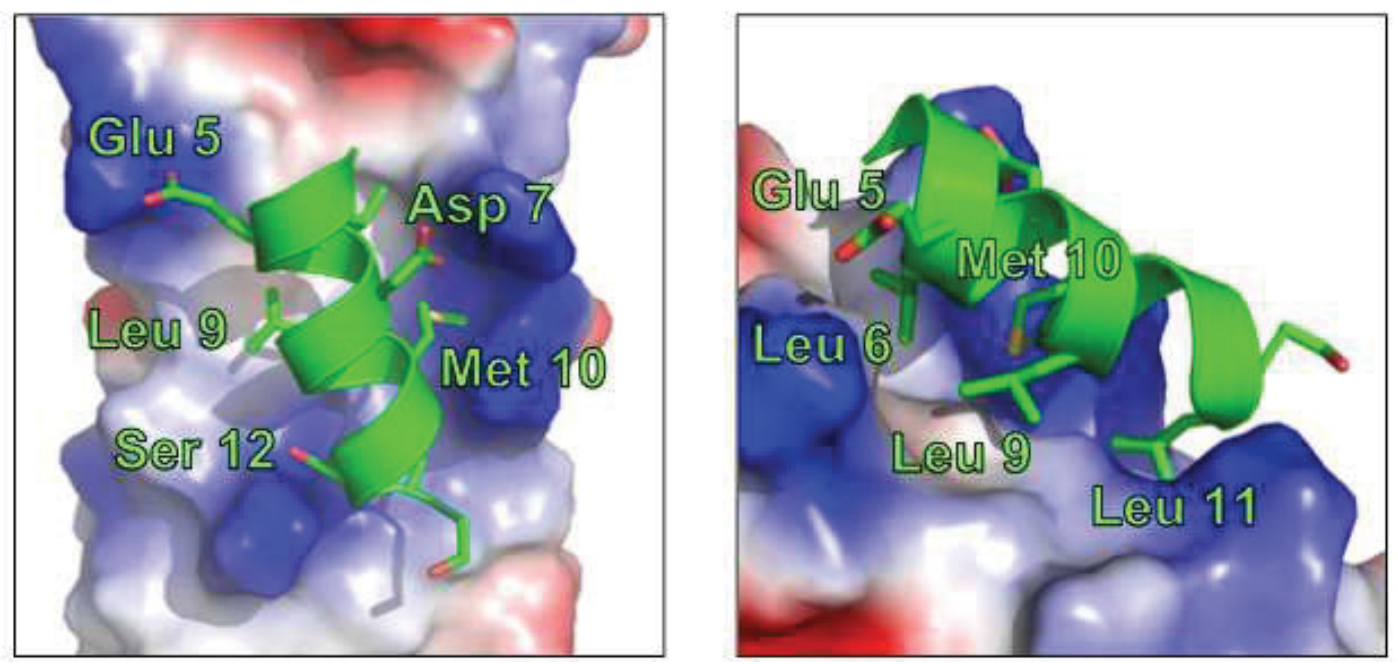

Figure 3-3. LD4 motif of paxillin binds to FAT of FAK driven by hydrophobic interaction.

Upon binding the FAT domain, LD4 forms an amphipathic alpha helix. The hydrophobic face of LD4 oriented toward the patch formed between helices H2 and H3 of FAT.

Residues Leu 6, Leu 9 and Leu 11 of LD4 contribute the major hydrophobic interaction addition with Met 10 for LD4 associating with the hydrophobic patch on the H2/H3 site of FAT. Charge residues Glu 5, Asp 7 and Ser 12 of LD4 form salt bridges with FAT to provide anchor points to position LD4 specifically on $\mathrm{H} 2 / \mathrm{H} 3$ 
characterized by observing no or minor contact, and observing the contact between linker of helical constrained LD4 and FAT-LD2. In the group one, we have constrained LD4 peptides on two positions, shown in Figure 3-4. The first one is the Arg4-Glu8 position; with the linker starting from the fourth residue from the N-terminal of the LD4 peptide, and cross-linked to the $i+4$ position residue side chains. Arg 4 (Arg 265 in full-length paxillin) at the fourth position in the LD4 peptide provided a convenient anchor point for side chain crosslinking due to its orientation away from the binding surface as well as its location has no contact of any side chain on the FAT-LD2 protein. Therefore, we crosslinked on Arg 4 and Glu 8 (Glu 269 in full-length paxillin) positions to constrain the LD4 peptide. In order to build the linker on this position, for lactam bridging, we replaced Arg 4 with Lysine, which has a longer side chain and can pair and link with the side chain of Glu 8. The charges carried by Lysine and Glutamic Acid will be neutralized after crosslinkage; For the hydrocarbon stapling, we use (S)-N-Fmoc-2-(4'-pentenyl) alanines, which have the additional artificial hydrocarbon side chain that can be covalently linked together, to replace both Arg 4 and Glu 8. Second is the Glu8-Ser12 position, and on this position we have built the linker close to the C-terminal of the LD4 peptide with potential minor contact with the FAT protein. We placed the linker on Glu8 and Ser 12 (Ser 273 in full-length paxillin). Glu 8 locates in the center of the LD4 peptide that is an ideal central anchor point, which we also used on group one. The second anchor point on position two, the Ser 12 has a potential interference with Lys 1002 of FAT, since the side chains of both residues are structurally close. However, the orientation of the side chains forms an angle that is not favorable to generate any chemical bond. Although, by mutating the Ser 12 in LD4, consequently, we may affect the binding specificity of LD4 peptide to the $\mathrm{H} 2 / \mathrm{H} 3$ site of FAT, but it will not eliminate the FAT/LD4 interaction. Hence, we can observe the difference on binding mode comparing the Arg4-Glu8 position.

In the second group, we selected three different positions, shown in Figure 3-5. The LD4 side chains on these positions all have been predicted to clash with the FAT surface. The selected anchor points of linkers in group two include the following positions: Asp 7 (Asp 268 in full-length paxillin) and Ala 11 (Ala 272 in full-length paxillin), in which the Asp 7 will form a salt bridge with Arg 962 of FAT; Glu 5 (Glu 265 in full-length paxillin) and Leu 9 (Leu269 in full-length paxillin), in which the Glu 5 will pair with the charged residue Lys 988 of FAT and Leu 9 contributes to the hydrophobic interaction; Leu 6 (Leu 266 in full-length paxillin) and Met 10 (Met 271 in full-length paxillin), in which both Leu 6 Met 10 locate on the hydrophobic surface of the peptide contributing to form the major hydrophobic interaction of the LD4 peptide to the H2/H3 hydrophobic patch of FAT. The group two constrained peptides open the ability to investigate the role of the linker on different positions with predicted interference with the FAT-LD2 surface or disrupting the charge residues pairing. Furthermore, it can be used to identify the essential residues in LD4/FAT interaction.

\subsubsection{Constrained LD4 Peptides Have an Enhanced Helix Propensity}

The LD4 motif is unstructured and highly dynamic in solution. When bound to the FAT domain, the LD4 motif forms an amphipathic alpha helix with its hydrophobic 


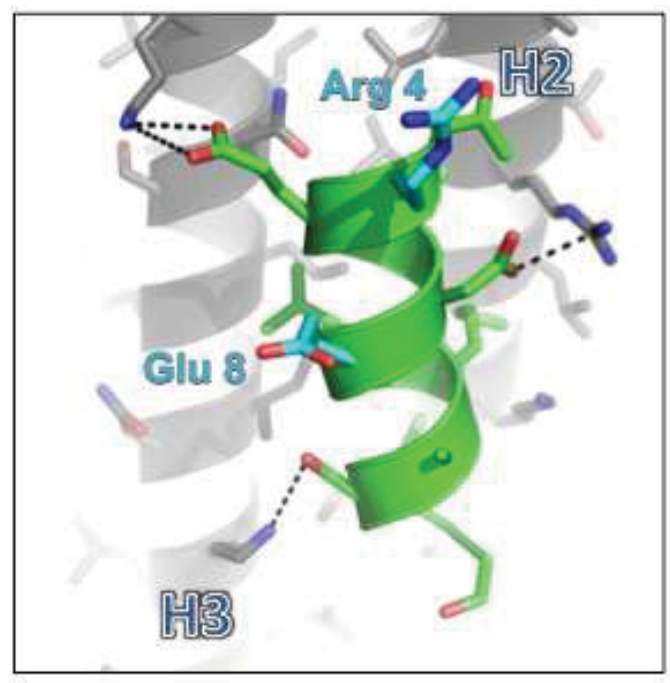

Arg4-Glu8 position

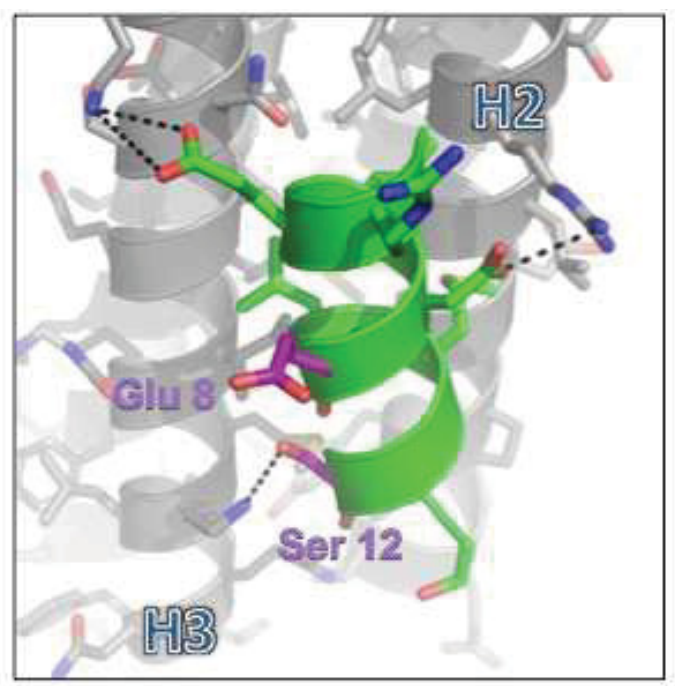

Glu8-Ser12 position

Figure 3-4. Group one helical constrained LD4 peptides with no or minor contact with FAT-LD2 surface.

Group one helical constrained LD4 peptide with a linker has contact with the FAT-LD2 surface on Arg4-Glu8 position, with the residues labeled by cyan. On the Glu8-Ser12 position the salt bridge formed by the Ser 12 side chain may be disrupted by placement of the linker. The residues involved in the linker position are colored purple. 


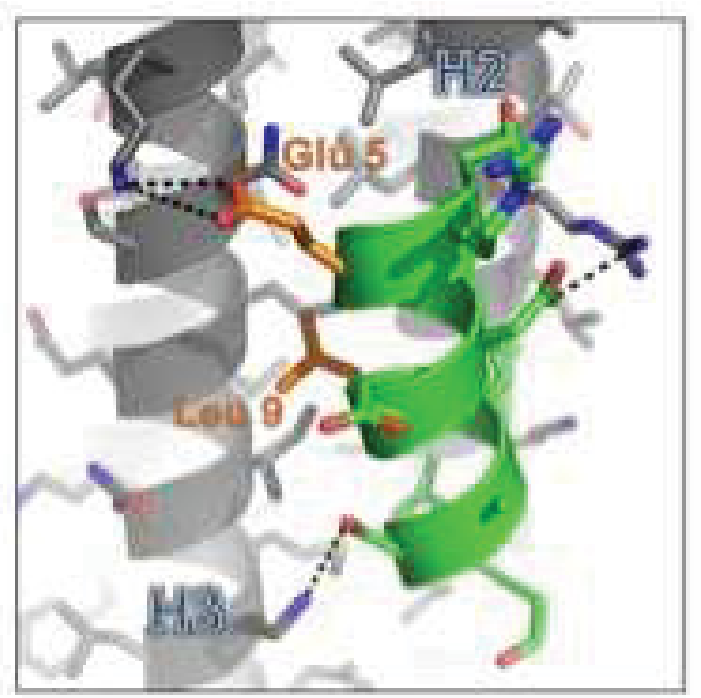

Glu5-Leu9 position

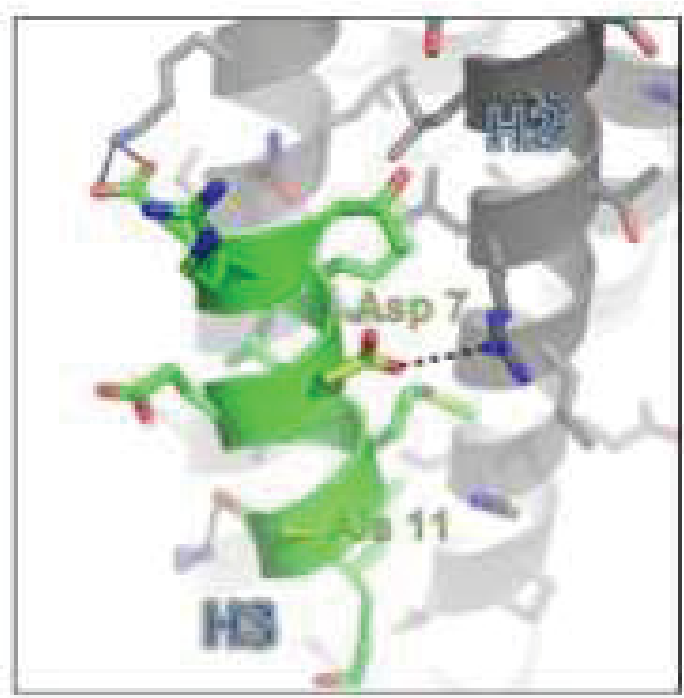

Asp7-Ala11 position

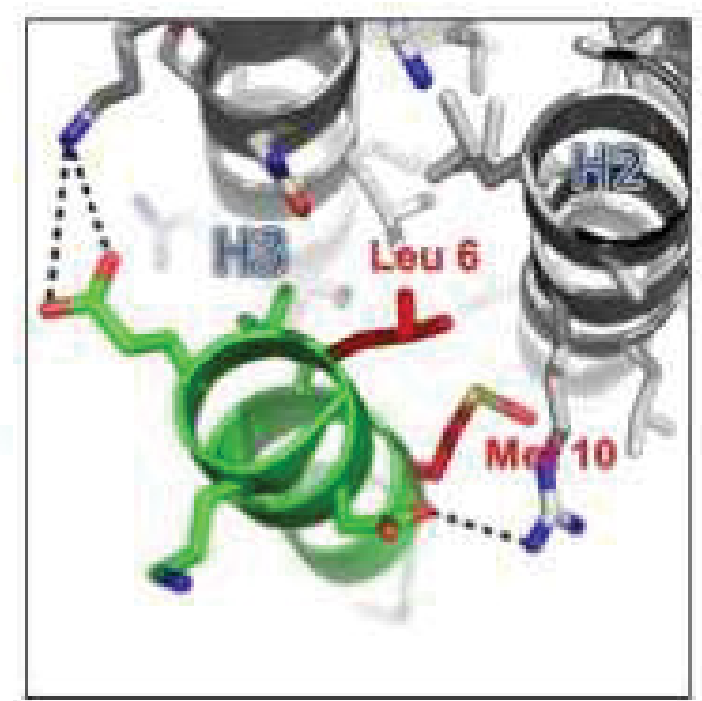

Leu6-Met10 position

Figure 3-5. Group two helical constrained LD4 with predicted interference of FAT-LD2 surface.

Group two helical constrained LD4 peptides have contact with the binding surface of FAT-LD3.On the Glu5-Leu9 position, with residues colored orange, the charged residue Glu 5 has been neutralized by cross-linking. On the Asp7-Ala11 position, with residues colored light green, the charge residue Asp7 has been neutralized by cross-linking. On the Leu6-Met10 position, with residues colored red, we placed the linker on the hydrophobic surface of the binding site on LD4. 
face oriented towards the hydrophobic patch formed between helices $\mathrm{H} 2$ and H3 of FAT, and positioned by forming salt bridges from charged residues. Entropy plays an important role in forming helical structure during the LD4/FAT interaction. By using the constraining approach to force the LD4 peptide into maintaining a helical structure the entropy penalty will be dramatically reduced, hence, the binding affinity of helical constrained LD4 to FAT will significantly increase. We produced both lactam bridge and hydrocarbon stapled LD4 peptides and validated the helical propensity by CD spectroscopy and NMR spectroscopy.

The CD spectra measurement has been performed to obtain secondary structure information by monitoring the far-UV spectral region (from $190 \mathrm{~nm}$ to $250 \mathrm{~nm}$ ). The chromophore at these wavelengths is the peptide bond, which means the signal arises when it is located in a folded environment. The secondary structures, such as alpha helix, beta sheet and coil structure, give rise to a characteristic shape and magnitude of each in CD spectrum. CD spectroscopy can determine which secondary structure dominated in the entire molecule. For coil structure the CD spectrum will appear as a single negative peak at $180 \mathrm{~nm}$, while the alpha helical structures show two signature negative peaks at both $210 \mathrm{~nm}$ and $220 \mathrm{~nm}$ and a positive peak at $190 \mathrm{~nm}$. Shown in Figure 3-6A, CD spectra suggest the WT-LD4 is an unstructured coil in solution and helical constrained peptide LD4-S12K forms the alpha helix. The data was further confirmed by Natural abundance HSQC, shown in Figure 3-6B. The HSQC spectrum of helical constrained LD4 peptide shows the well dispersed peaks with equal size that indicates well folded structure compared with WT-LD4.

\subsubsection{Helical Constrained LD4 Peptides Show Enhanced Binding Affinity to FAT- LD2}

The binding affinity of the helical constrained LD4 peptides bound to FAT-LD2 was measured using ITC. In order to compare the difference in binding affinity, we first examined WT LD4 and obtained the binding affinity of $\mathrm{K}_{\mathrm{D}}$ at approximate to $27 \mu \mathrm{M}$. In comparison, both the Lactam-bridged and Hydrocarbon-stapled LD4 peptides from two groups were examined by titrating cross-linked peptides against FAT-LD2. Results are shown in Table 3-1. The experimental data shows the increase of the binding affinity of both helical constrained LD4 peptides comparing with WT LD4 peptide except in a few cases.

We characterized the helical constrained LD4 peptides in two groups, based on the positions of the linker, and whether we observed interference of linker with FAT surface. Summarized from the ITC data: in group one, which the linkers of peptides have no contact with FAT, we observed a best 4.5 fold increase on binding affinity comparing with WT LD4 peptide; In group two, in which the linkers of peptides either interfere with the FAT surface preventing salt bridge formation, or they disrupt the hydrophobic interaction, showed weak or no binding. However, the LD4-L6A peptide (Leu6-Ala11 position) shows a significantly 30 -fold enhanced binding affinity to FAT-LD2 with a KD of $0.8 \mu \mathrm{M}$. The key factors for the association of LD4 to FAT are: hydrophobic 

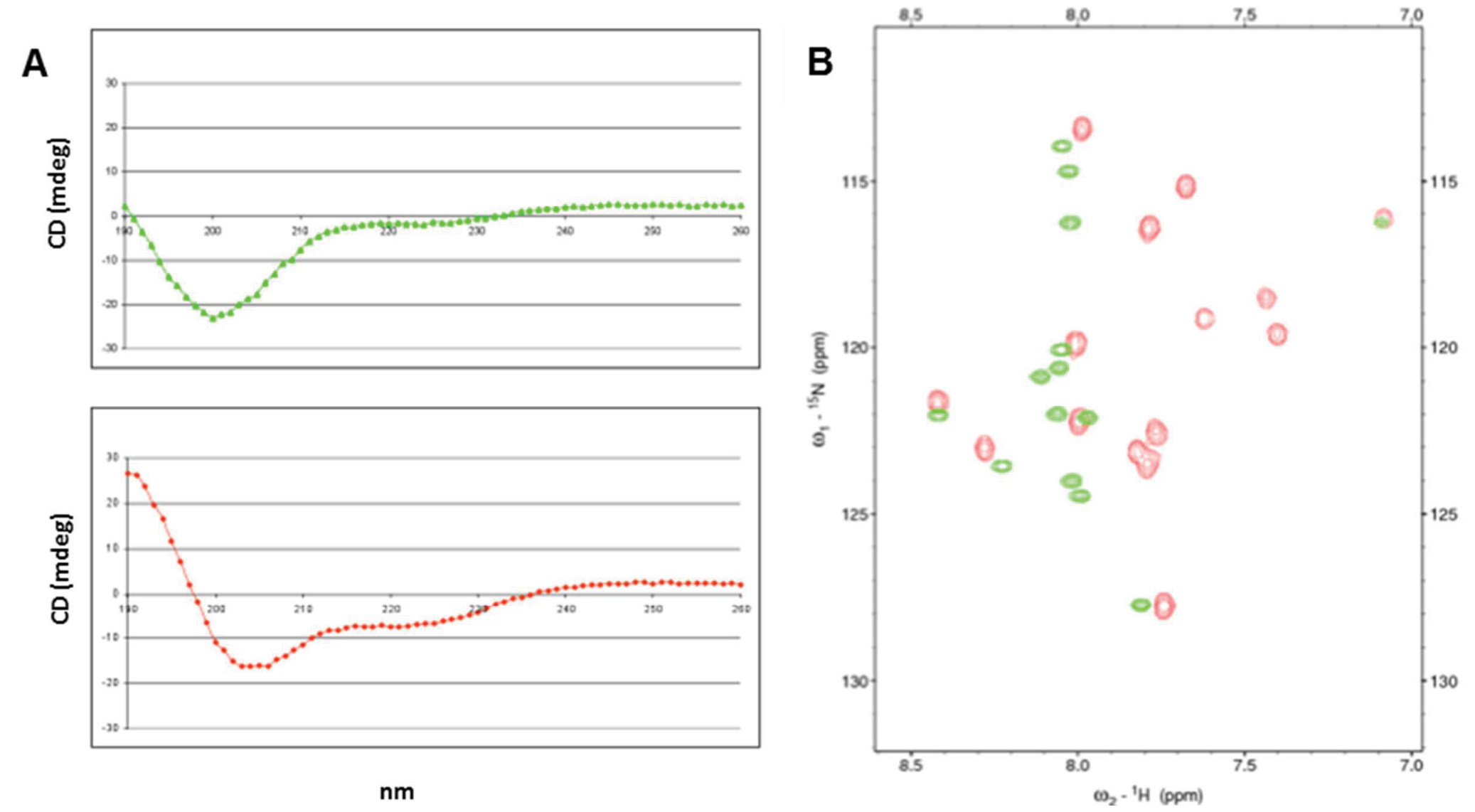

Figure 3-6. Helical constrained LD4 peptide shows enhanced helix propensity in solution.

Green color represents the WT-LD4 peptides and red color represents the LD4-S12K. CD spectra show the enhancement of the alpha helix signature peaks on $200 \mathrm{~nm}$ and $220 \mathrm{~nm}$ of LD4-S12K compared with WT-LD4. The NMR HSQC data also agrees with the conclusion. The spectrum of LD4-S12K shows better dispersed peaks comparing with LD4 indicating the helical constrained peptide adopts better folding. 
Table 3-1. Binding affinity of LD4 peptides to FAT-LD2.

\begin{tabular}{|c|c|c|c|}
\hline Numbers & Peptides & $\mathbf{K D}(\mu \mathrm{M})$ & Sequence \\
\hline 1 & LD4 & 27.7 & SATRELDELMASLSD \\
\hline 2 & $\mathrm{R} 4 \mathrm{~A}-\mathrm{C}$ & 6.2 & SATAELDALMASLSD \\
\hline 3 & $\mathrm{R} 4 \mathrm{~K}-\mathrm{L}$ & 10.3 & SATKEELDELMASLSD \\
\hline 4 & E5A-C & Weak & SATRALDEAMASLSD \\
\hline 5 & L9K-L & No & SATRELDEKMASLSD \\
\hline 6 & L6A-C & 0.8 & SATREADELAASLSD \\
\hline 7 & D7A-C & 7.1 & SATRELAELMASLSD \\
\hline 8 & S12A-C & 11.4 & SATRELDALMAALSD \\
\hline 9 & S12K-L & 5.9 & SATRELDELMAKLSD \\
\hline
\end{tabular}


interaction for binding LD4 onto the hydrophobic patch of $\mathrm{H} 2 / \mathrm{H} 3$ of FAT; Forming the salt bridge by charge residues for positioning the LD4 and enhancing the interaction; Maintaining the helical structure and dynamic of LD4 peptide to reduce the entropy. We believe the hydrophobic hydrocarbon linker on the contact surface of LD4-L6A, indeed, increases hydrophobic interactions between the peptide and FAT protein. Also when placing the linker on Leu6-Ala11 position the LD4-L6A avoids disturbing the salt bridge formation by maintaining all the side chains of the charged residues. Finally the linker placed in the center of LD4-L6A largely enhanced the helix propensity. From all these aspects we interpret a significant enhanced binding affinity of LD4-L6A to FAT-LD2.

\subsubsection{Enhanced Association of Cross-linked LD4 Peptides with FAT-LD2 Validated by Chemical Shift Perturbation}

The binding affinity of the helical constrained LD4 peptide for FAT-LD2 was determined from ITC titration plots as shown above. Helical constrained LD4 peptides show the enhancement on association with FAT-LD2. Mapping the binding site of modified LD4 peptides is limited by the ITC method. For validating the interaction and mapping the binding site of modified LD4 with FAT-LD2 we introduce NMR spectroscopy to monitor the chemical shift perturbation (CSP). Comparison of the spectrum of WT-LD4 titrated into FAT-LD2 with the spectra of helical constrained LD4 peptides titrated into FAT-LD2 has been performed for mapping the binding site. Then the residues with the maximum CSPs on the binding site of $\mathrm{H} 2 / \mathrm{H} 3$ were selected to determine the binding affinity from NMR titration experiments.

NMR is a useful and sensitive method for determining specific protein-ligand interactions in solution. With the backbone assignment and specific CSP pattern of titrated ligand, the NMR spectrum can be used as a footprint to describe the binding site of the target ligand. Shown in Figure 3-7, superimposed spectra of WT-LD4 and crosslinked LD4 peptides titrating into FAT-LD2 shows the identical CSP patterns. The CSPs data suggest the cross-linked LD4 peptides associate with FAT-LD2 binding to the same site as WT-LD4.

For validating the ITC based binding affinity of cross-linked LD4 peptides to FAT-LD2 by NMR the residues Ala 942 and Ala 976 have been selected as representing the maximum CSPs, shown in Figure 3-8. The binding affinities obtained from CSP plots from selected residues for WT-LD4 calculated around $400 \mathrm{uM}$ while the representative helical constrained peptide LD4-L6A at a $\mathrm{K}_{\mathrm{D}}$ around $1.2 \mathrm{uM}$.. The NMR is

a useful method for determining interactions of protein and ligand in solution; however, it has been known that it is not the ideal method for calculating binding affinity. Due to the fact that averaging effects during NMR experiments generate a large systemic error in calculating. $\mathrm{K}_{\mathrm{D}}$ values (158). Therefore we only use the CSP based binding affinity data as references to validate the enchantment of $\mathrm{K}_{\mathrm{D}}$ of cross-linked LD4 measured using the ITC method. Together the NMR CSP data consistent with our previous observation from the ITC study. 


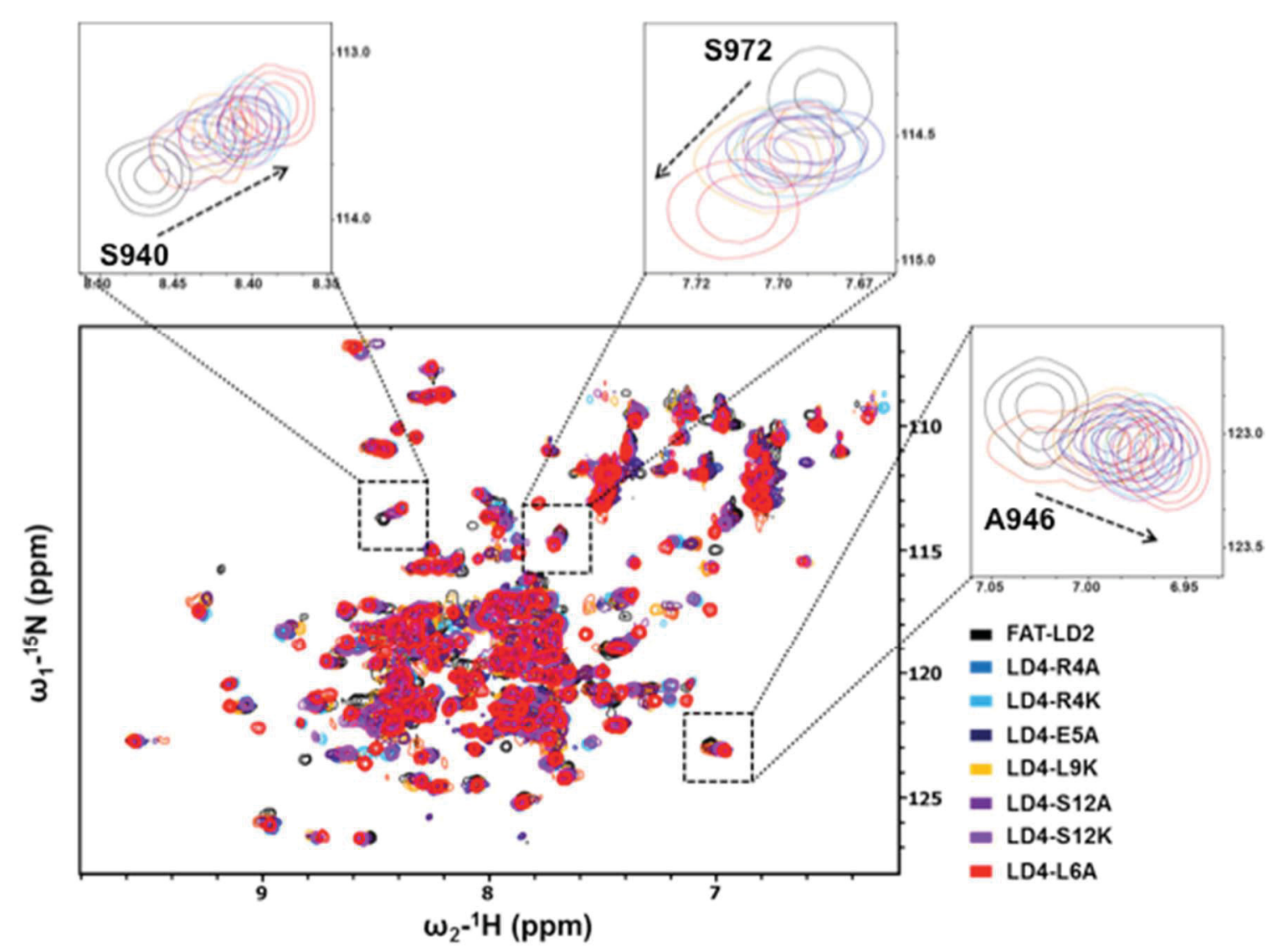

Figure 3-7. LD4-L6A significantly enhanced binding to FAT-LD2 based on chemical shift perturbation.

Chemical shift perturbation of LD4 and helical constrained peptides measured by ${ }^{15} \mathrm{~N}$ HSQC have been plotted and superimposed. Observed from spectra the CSPs pattern and directions are identical for all peptides titrating into FAT-LD2. Data suggest the helical constrained peptides share the same binding mode as WT-LD4. 
Figure 3-8. LD4-L6A has higher binding affinity to FAT-LD2 comparing with WTLD4.

NMR based $K_{D}$ measurements have been performed by titrating LD4-L6A into FATLD2, and LD4 has been included as control. Different color represents the ratio between protein and ligand, from a ratio 1:0 to 1:1.5. The residues Ala 946 and Ala 972 involved in the binding site with the maxima chemical shift perturbation have been selected to plot and calculate $\mathrm{K}_{\mathrm{D}}$ volume of LD4 and LD4-L6A. Data shows LD4-L6A achieved saturation at the ratio 1:1.5 (FAT-LD2:LD4-L6A), while the LD4 does not, indicating a significant stronger binding affinity of LD4-L6A comparing with LD4. The $\mathrm{K}_{\mathrm{D}}$ calculated for LD4 based on CSPs is $400 \mathrm{uM}$, when LD4-L6A is $1.2 \mathrm{uM}$. 

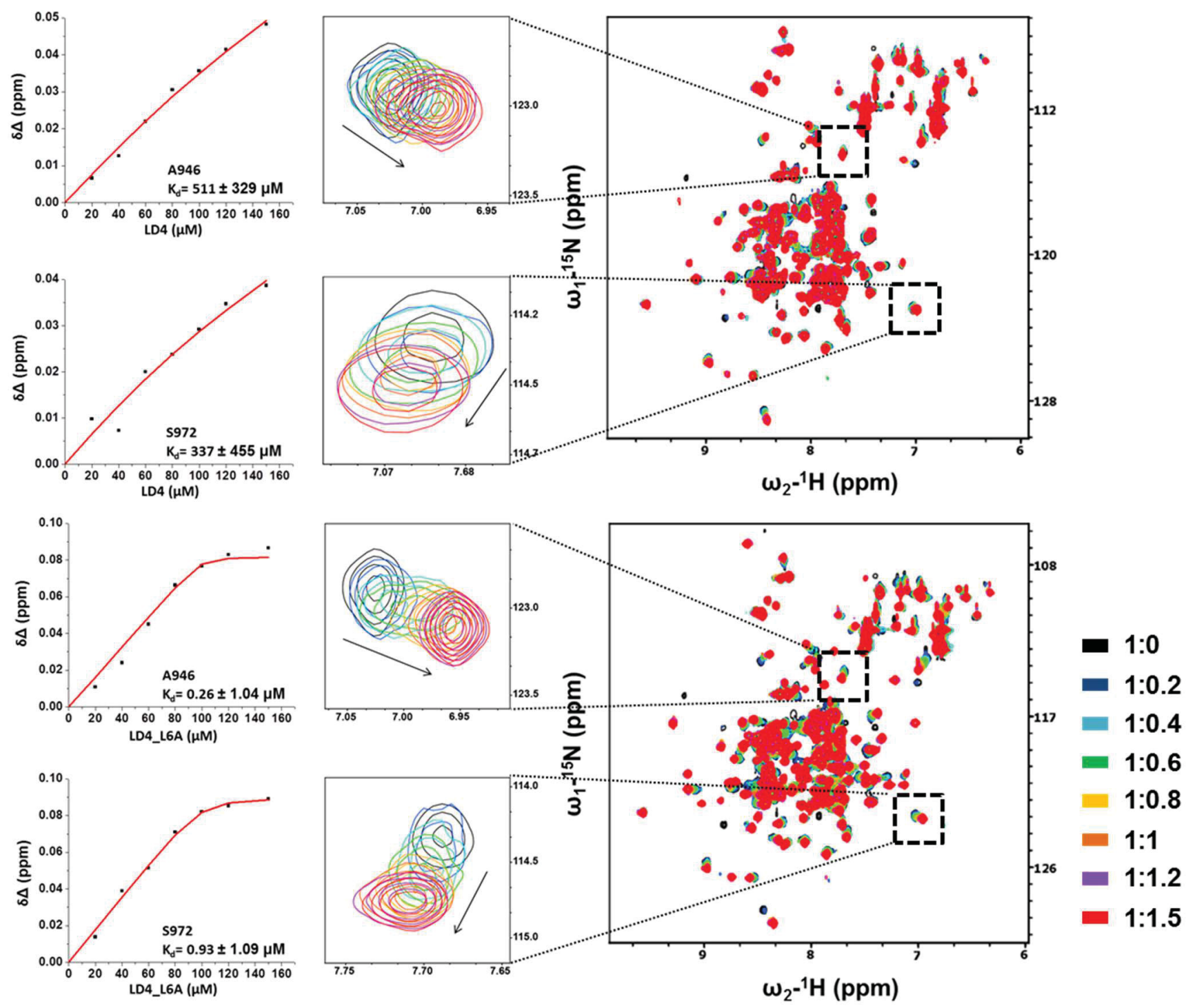


\subsubsection{Helical Constrained LD4 Peptides Can Compete with a WT-LD4 Peptide as Inhibitor}

Both the ITC and NMR measurements show constraining helical structure and increasing the helix propensity enhances the association of LD4 to FAT-LD4. Especially the LD4-L6A peptide that combines the improved helix propensity and hydrophobicity to achieve approximates 30 -fold in binding affinity to FAT-LD2. The LD4-L6A potentially can be used as small molecular inhibitor for targeting FAK/paxillin interaction in FAs. Therefore we tested whether LD4-L6A has the ability to compete with WT-LD4 binding to FAT-LD2. We preformed the competitive binding assay using the fluorescence polarization (FP) method. In a regular FP assay we titrate ligand into protein in order to obtain the $\mathrm{K}_{\mathrm{D}}$ of the target ligand. Different from the regular FP assay for $\mathrm{K}_{\mathrm{D}}$ plotting the competitive binding assay measures apparent $\mathrm{K}_{\mathrm{D}}$ of a reference ligand to calculate the $\mathrm{K}_{\mathrm{I}}$ of the target ligand, which is added to the sample solution as a competitor. In our case we use WT-LD4 as reference ligand and use LD4-L6A as competitor to calculate the $\mathrm{K}_{\mathrm{I}}$ of LD4-L6A. As a comparison we measured $\mathrm{K}_{\mathrm{I}}$ for WT-LD4, shown in Figure 3-9. From the results we observed a larger apparent $\mathrm{K}_{\mathrm{D}}$ value of addition of LD4-L6A as competitor than addition of LD4, and the $\mathrm{K}_{\mathrm{I}}$ of LD4-L6A calculated from apparent $\mathrm{K}_{\mathrm{D}}$ is stronger than LD4. Data suggests LD4-L6A has the ability to competitively bind to FAT-LD2 with WT-LD4.

We further investigated whether LD4-L6A can break down the FAT-LD2/LD4 complex by an inhibitory FP assay. Florescent labeled FAM-LD4 peptides has been mixed with FAT-LD2 protein to form a stable complex. Then cross-linked LD4 peptide including LD4-L6A has been titrated into the sample solution, shown in Figure 3-10. Data shows LD4-L6A achieves $50 \%$ inhibition at approximate $6 \mu \mathrm{M}$ while the WT-LD4 works at at approximately $80 \mu \mathrm{M}$ as a control. The second best inhibitor is LD4-R4A that achieves $50 \%$ inhibition at approximately $25 \mu \mathrm{M}$. The inhibitory FP data confirmed our previous data that LD4-L6A has a significant enhancement on binding affinity to FATLD2. LD4-L6A can efficiently compete with LD4 binding with FAT-LD2 and achieve the ability to disrupt FAT/LD4 complex.

\subsection{Discussion}

Focal Adhesion Kinase localizes in focal adhesions that are overexpressed in many tumors and have been shown to be involved in the regulation of a variety of cell processes that are important in tumorigenesis such as cell growth, survival and migration. 1-5 Our particular interest is its role in the formation and disassembly (turnover) of focal adhesion contacts as this is a key mechanism in cancer cells undergoing metastasis. Hence, regulating the activity of FAK in FAs may control the tumorigenesis. The FAT domain localized at the C-terminal region of FAK consists of a right turn elongated four-helix bundle function as the protein-protein interaction domain. FAT domain is maintained by hydrophobic interactions. The helices $\mathrm{H} 1$ and $\mathrm{H} 4$, and helices $\mathrm{H} 2$ and $\mathrm{H} 4$ compose two large hydrophobic patches respectively that response for FAK FAs localization through the interaction of paxillin. 

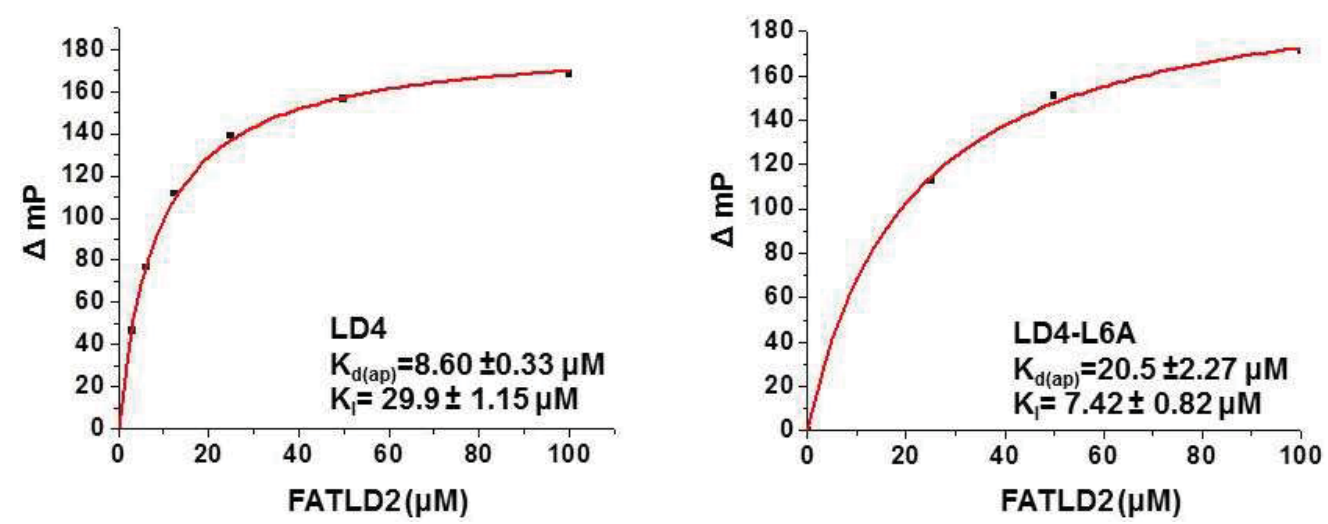

Figure 3-9. LD4-L6A binds to FAT-LD2 competitively with WT-LD4.

FAT-LD2 protein was titrated with FAM-LD4 addition with the competitor, in this case LD4-L6A and WT-LD4 as control, to plot the binding curve for obtaining the Apparent $\mathrm{K}_{\mathrm{D}}$ of LD4 presenting with LD4-L6A as competitor and LD4 as control, respectively. $\mathrm{K}_{\mathrm{I}}$ of LD4-L6A and LD4 then were calculated from apparent $\mathrm{K}_{\mathrm{D}}$. Results indicate the LD4L6A can significantly compete with LD4 to bind with FAT-LD2. 


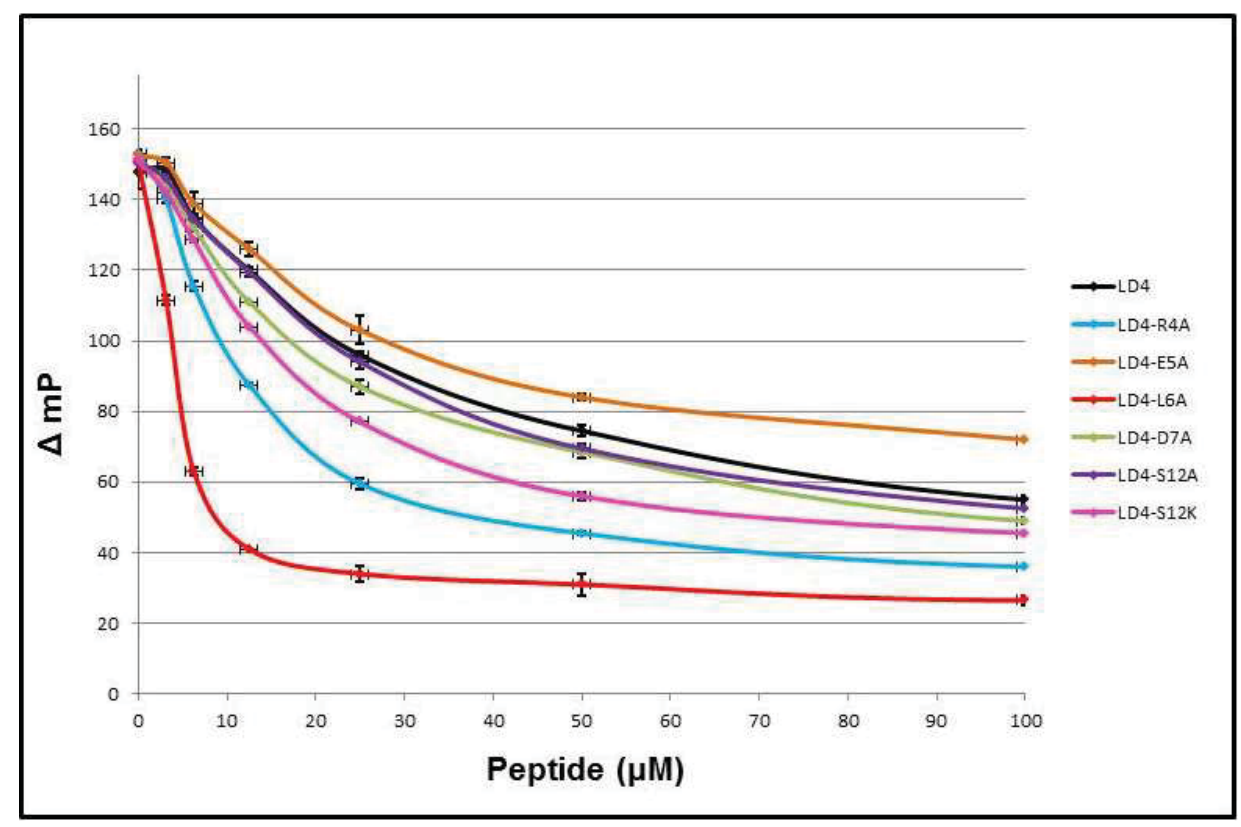

Figure 3-10. Helical constrained LD4 peptides can disrupt the FAT-LD2/LD4 complex.

FAT-LD2 was added into FAM-LD4 and incubated for 10 minute for achieving a stable complex. Then helical constrained peptide was titrated into the sample solution to test the ability of inhibiting FAM-LD4/ FAT-LD2 association. LD4-L6A reached 50\% inhibition at approximate $6 \mathrm{uM}$, in addition the second best constrained peptide LD4-R4A reached $50 \%$ at approximate $25 \mathrm{uM}$. 
There are five LD motifs in the N-terminal half of paxillin. Those LD motifs are highly conserved throughout the paxillin superfamily. The LD motifs of paxillin bind to many different molecules. The flexible nature of the N-terminal domain likely facilitates paxillin's ability to interact with different binding partners by using different LD motifs. Indeed, the LD2 and LD4 motifs appear to bind to the FAT domain at two completely different sites simultaneously. The reason for multiple binding sites on FAT for paxillin interaction remains unclear. Perhaps multiple binding sites add specificity for various binding partners of paxillin utilizing common LD motifs. Although paxillin interacts with different molecules, FAK is one of its major binding partners The FAK-paxillin complex plays an important role in focal adhesions [6]. In a migrating cell, the dynamics of focal adhesion formation and disassembly are essential for cell movement. The dissociation of the FAK-paxillin complex could lead to the breakdown of focal adhesions, and such dissociation of the FAK-paxillin complex is most likely achieved by inhibition of one of the two LD motifs from binding to the FAT domain. Both LD motifs can interact with many other molecules[6]. Because of the weak interaction between the FAT domain and the individual LD motifs, a disassembly signal such as an LD4 binding molecule, would be able to bind a FAT bound LD4 thus disassociating it from the FAT domain. The consequence of such a binding event would be a significant destabilization of the FAKpaxillin interaction and the eventual break down of the complex. Indeed, to regulate the activity of FAK in FAs we proposed to use a cross-linking approach to synthesize a helically constrained LD4 peptidomemtic that binds FAT with increased affinity to inhibit paxillin binding.

In order to form stable complex both LD2 and LD4 motifs binding with sites $\mathrm{H} 1 / \mathrm{H} 4$ and $\mathrm{H} 2 / \mathrm{H} 3$ of FAT are required. The LD2 motif can freely bind to the $\mathrm{H} 1 / \mathrm{H} 4$ site or the $\mathrm{H} 2 / \mathrm{H} 3$ site while the LD4 motif can only bind to $\mathrm{H} 2 / \mathrm{H} 3$ site. The difference may be caused by the helix propensities between these two motifs. LD motifs form amphipathic helices based on the amino acid sequences and the difference in helix propensities may due to their helix capping motifs. The LD2 helix ends at a proline residue, which is known to be a strong helix breaker in addition to glycine. In contrast, the LD4 sequence does not contain either a proline or glycine at the C-terminal end of the helix. Previous studies from our lab have shown that LD4 exists as a random coil in solution, folding into a helix upon binding to the $\mathrm{H} 2 / \mathrm{H} 3$ surface of FAT. Thus, in the case of LD4, it is likely that interacting residues within the $\mathrm{H} 2-\mathrm{H} 3$ binding pocket on FAT provide the hydrogen bonds responsible for stabilizing the $\mathrm{C}$-terminal end of the helix instead. The structures indicate that the residues K956 and K1003 within the $\mathrm{H} 2 / \mathrm{H} 3$ binding pocket of FAT form hydrogen bonds with the backbone carbonyls of residues Leu 274 and Ser 273 of the LD4 motif. Inspection of the H1/H4 binding pocket reveals that there are no residues in the corresponding location that could provide such stabilizing hydrogen bonds. This may explain in part the relative selectivity in binding of the LD4 motif.

Since the LD4 has the ability to specifically bind to the H2/H3 site of FAT, we selected LD4 peptide to cross-link and use the helical constrained LD4 peptides as an inhibitor to target the paxillin/FAK interaction. In this study we synthesized two types of helical constrained peptide by lactam bridging and hydrocarbon stapling approaches. 
Overall the cross-linked peptides gain an increase on binding affinity for FAT. Furthermore, placing the linker at different positions on LD4 peptides opens the opportunity for investigating the key residues of LD4 associating with FAT. Together we identify the potential of LD4-L6A as the inhibitor targeting FAK/paxillin interaction among all the helical constrained peptides. The LD4-L6A adopts 30-fold enhancement of binding affinity to FAT while others cross-linked LD4 peptides enhanced at average 4fold. Natural LD4 peptide has been known to adopt a coil like structure in solution and forms alpha helix upon binding. The binding of LD4 to FAT is driven mainly by hydrophobic contact between both the peptide hydrophobic surface and $\mathrm{H} 2 / \mathrm{H} 3$ hydrophobic patch of FAT. This association has been further stablized by paring of the charge residues on LD4 and FAT. Our LD4-L6A peptide has enhanced helix propensity by cross-linking the Leu 6 and Ala 10 residues, which are on the center region of the peptide that gives the best structural constraining. The hydrocarbon linker also contributes to the interaction of LD4-L6A to FAT-LD2 by increasing the hydrophobicity. Finally, the hydrocarbon linker is far away from all charged residues on LD4 avoiding the interference of salt bridge formation. Thus, the binding affinity of LD4-L6A has been significantly enhanced. Furthermore, competitive binding assay and inhibitory binding assay has been performed to evaluate LD4-L6A as an inhibitor. Since from the data LD4L6A peptides bind specifically to the $\mathrm{H} 2 / \mathrm{H} 3$ pocket of FAT competing with WT-LD4, it should be possible to use LD4-L6A and its derivatives to elucidate the signaling pathways that are impacted by binding of LD2 and LD4 peptides. 


\section{CHAPTER 4. STRUCTURE DETERMINATION OF P130CAS CAS FAMILY C- TERMINAL HOMOLOGY DOMAIN}

\subsection{Introduction}

Cell-ECM attachment transmits extracellular signals from the ECM into the cytosol, which are important for cell growth, survival and migration. This process is mainly performed by integrin family members and contributes to the formation of the Focal Adhesions (FAs). P130cas is a Src substrate localized in Focal Adhesions (FAs) that functions in integrin signaling to promote cell motility, invasion, proliferation and survival. It is a scaffold molecule containing an N-terminal SH3 domain, a substrate domain (SD) and a Cas family C-terminal homology domain (CCHD), but lacking enzymatic activity. The SH3 domain of p130Cas can directly interact with Focal Adhesion Kinase (FAK), which is a non-receptor tyrosine kinase that prominently locates in FAs. FAK serves the dual functions of recruiting p130Cas to FAs and recruiting Src kinase to phosphorylate the p130Cas SD to promote downstream signaling. P130Cas targeting to focal adhesions and undergoing tyrosine phosphorylation in responding to adhesion signals plays an important role in cell motility, invasion and survival. Mice lacking p130Cas revealed embryonic lethality due to defects associated with the disorganized actin cytoskeleton [145]. In addition, p130Cas is also up regulated in cancer cells. My long-term goal is to understand how p130Cas functions and mediates integrin signaling

Although the importance of p130Cas localization and phosphorylation is well established, how it targets FAs has not been elucidated. Previous studies demonstrated that, the N-terminal SH3 domain is critical for p130Cas localization through interacting with FAK. SH3 deleted p130Cas revealed a six-fold decrease in FAs localization ability compared with wild-type [145]. In addition, deletion of the CCHD of p130Cas also leads to three-fold decrease of FA localization ability. The C-terminal region of p130Cas has been proposed as a Cas family homology domain, which may adopt a similar folding formation to the FAK C-terminal Focal Adhesion Targeting (FAT) domain. The nature of the target protein for the p130Cas $\mathrm{CCH}$ domain in promoting FAs localization is unclear. Here I propose Paxillin, which plays a major role in targeting FAK to FAs through interaction with the FAK C-terminal FAT domain, promotes the $\mathrm{CCH}$ domain mediated localization of p130Cas to FAs.

In order to better understand p130Cas localization mechanism and its interplay with integrin signaling, we propose to solve the complex structure of p130Cas CCHD with Paxillin LD motifs. Nuclear Magnetic Resonance (NMR) Spectroscopy was performed for evaluating the quality of protein and for backbone assignment. A complex structure of CCHD associated with LD motif was solved by X-ray crystallography. 


\subsection{Experiment Procedures}

\subsubsection{Protein Expression and Purification}

Mouse p130Cas cDNA was a gift from Dr. Schlaepfer, Scripps Research Institute. We generated series of constructs based on secondary structure predictions containing the target p130Cas ${ }^{738-874}$ (residues 738 to 874 ). By testing the solubility and stability, we selected the $\mathrm{p} 130 \mathrm{Cas}^{665-874}$ construct $\mathrm{p} 130 \mathrm{Cas}^{738-874}$ (residues 665 to 874 ). This construct was containing the full length CCHD sequence (residues 738 to 874) and an N-terminal 69 amino acids extension (residues 665 to 738), which dramatically solubilized the protein in solution, as shown in Figure 4-1. In addition, we mutated the Glycine ${ }^{735}$ to Arginine, Arginine ${ }^{736}$ to Glycine and Threonine ${ }^{737}$ to Serine, which created a thrombin cleavage site in between CCHD and the N-terminal extension. The Thrombin cleavage site was used to remove the N-terminal p130Cas ${ }^{665-738}$ elongation during protein purification. We subcloned the p130Cas ${ }^{665-874}$ construct into a pET28 vector (Novagen) fused with an N-terminal 6x His-tag. Incorporation of paxillin into $\mathrm{pET} 28 \mathrm{a}$ was verified by sequencing using the T7 terminator primer. His-tagged p130Cas ${ }^{665-874}$ construct was transformed into expression Escherichia coli (E.coli) strain BL21 (DE3). E.coli containing the p130Cas ${ }^{665-874}$ construct was cultured in $100 \mathrm{ml} \mathrm{LB}$ medium with $30 \mu$ $\mathrm{g} / \mathrm{ml} \mathrm{Kanamycin} \mathrm{at} 37^{\circ} \mathrm{C}$ in a rotary shaker at 250 revolutions per minute (rpm) for overnight. Then inoculated $10 \mathrm{ml}$ starting culture into each of six $1 \mathrm{~L}$ aliquots of $\mathrm{LB}$ medium containing $30 \mu \mathrm{g} / \mathrm{ml}$, and the $1 \mathrm{~L}$ cultures were allowed to grow at $37^{\circ} \mathrm{C}$ at 250 $\mathrm{rpm}$ for four to five hours until the $\mathrm{OD}_{600}$ reached 0.6 to 0.8 . Protein expression was initiated through the induction of T7 RNA polymerase by adding isopropyl $\beta$-Dthiogalactopyranoside (IPTG) to a final concentration of $1 \mathrm{mM}$. Induced cultures were then incubated at a reduced temperature and shaker speed of $18^{\circ} \mathrm{C}$ and $200 \mathrm{rpm}$ respectively for an overnight allowing protein expression. Isotope labeled protein was prepared using morpholinepropanesulfonic (MOPOS) acid-buffered medium containing ${ }^{15} \mathrm{NH}_{4} \mathrm{Cl}(1 \mathrm{~g} /$ liter $)$ and ${ }^{13} \mathrm{C}_{6}$-glucose $(3.6 \mathrm{~g} /$ liter $)$. The purification of $\mathrm{p} 130 \mathrm{Cas}^{665-874}$ followed the standard protein purification procedures, including $\mathrm{Ni}^{2+}$ histidine affinity chromatography, thrombin cleavage, and size exclusion chromatography. Cell lysates from 6 liters of overnight cultures were loaded manually on a pre-packed $5 \mathrm{ml} \mathrm{Ni}^{2+}$ histidine affinity column with a flow rate of approximately $1 \mathrm{ml}$ per minute. The resins were then washed with 10-column volumes of lysis buffer and the His- p130Cas ${ }^{665-874}$ was eluted with $50 \mathrm{mM}$ phosphate buffer, $500 \mathrm{mM}$ sodium chloride, 0.02\% 3-[(3Cholamidopropyl) dimethylammonio]-1-propanesulfonate (CHSPS), and containing 200 $\mathrm{mM}$ imidazole. The purified p130Cas ${ }^{665-874}$ protein was dialyzed against $50 \mathrm{mM}$ 4-(2hydroxyethyl)-1-piperazineethanesulfonic acid(HEPES), $\mathrm{pH} 7.8,300 \mathrm{mM}$ potassium chloride and $0.02 \%$ CHSPS. Thrombin was added to His- 130 Cas $^{665-874}$ with a mass ratio of $1: 100$ and $\mathrm{CaCl}_{2}$ to a final concentration of $2.5 \mathrm{mM}$ for overnight cleavage at 4 ${ }^{\circ} \mathrm{C}$. His-tag along with the N-terminal extension p130Cas ${ }^{665-737}$ were removed and in the mixture with target region p130Cas ${ }^{737-874}$. The mixture was concentrated and added the LD1 peptide to a molar ratio 1 to 5 during the concentration procedure to enhance and stablize the CCHD/LD1 complex. Concentrated protein was further purified with gel 


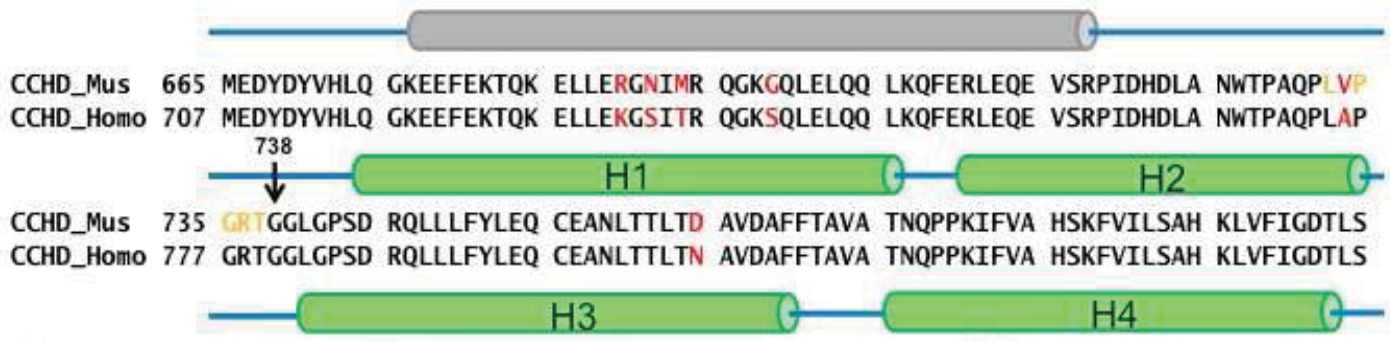

CCHD_MUS 805 RQAKAADVRS QVTHYSNLLC DLLRGIVATT KAAALQYPSP SAAQDMVDRV KELGHSTQQF RRVLGQLAAA CCHD_HOMO 847 RQAKAADVRS QVTHYSNLLC DLLRGIVATT KAAALQYPSP SAAQDMVERV KELGHSTQQF RRVLGQLAAA

Figure 4-1. Sequence analysis and secondary structure prediction of CCHD. Secondary structure prediction of the C-terminal region of p130Cas shows the CCHD consists of four alpha helixes colored in green and a potential alpha helix at its Nterminal colored in gray. This additional helix can stabilize the CCHD protein. Construct p130Cas ${ }^{665-874}$ containing the p130Cas ${ }^{738-874}$ (CCHD) and the additional helix region p130Cas ${ }^{665-737}$ has been generated. Sequence colored in yellow has been mutated to a secondary thrombin cleavage site for removing the p130Cas ${ }^{665-737}$ during purification. Sequence colored in red shows the unconserved residues comparing the mouse and human species. It shows only two residues are different in CCHD from human to mouse. 
filtration size exclusion chromatography (26/60 Superdex 75 HR column, GE Healthcare), using an AKTA FPLC system. The final protein buffer used for all NMR experiments, biochemical studies, and X-ray crystallization was $20 \mathrm{mM}$ HEPES, $100 \mathrm{mM}$ potassium chloride, at $\mathrm{pH}$ 7.5.

\subsubsection{Peptide Synthesis}

Paxillin derived peptides LD1 (Gallus; residues 1 to 15), peptides LD2 (Gallus; residues 139 to 162), LD3 (Gallus; residues 217 to 237) peptides LD4 (Gallus; residues 261 to 276), and LD5 (Gallus; residues 29 to 324) were chemically synthesized and purified by high pressure liquid chromatography (HPLC) at the Hartwell Center of Bioinformatics and Biotechnology (St. Jude Children's Research Hospital). All peptide stocks were prepared at a concentration of $2 \mathrm{mM}$ in $50 \mathrm{mM}$ HEPES, $\mathrm{pH} 7.8,300 \mathrm{mM}$ potassium chloride and $0.02 \%$ CHAPS.

\subsubsection{Isothermal Titration Calorimetry Studies}

Isothermal Titration Calorimetry experiments were performed using a MicroCal Auto-ITC200 instrument (GE Healthcare). The sample cell of the calorimeter was loaded CCHD in a concentration of $0.025 \mathrm{mM}$ in $50 \mathrm{mM}$ HEPES, pH 7.8, $300 \mathrm{mM}$ potassium chloride and $0.02 \%$ CHAPS. The syringe of the calorimeter was loaded LD peptides in a concentration of $0.25 \mathrm{mM}$ in the same buffer of CCHD. Prepared solutions were centrifuged for $10 \mathrm{~min}$ at $14000 \mathrm{x}$ g for degassing. All the titration experiments were performed at $25^{\circ} \mathrm{C}$ with injection volumes of $2 \mu \mathrm{l}$ and a spacing of $120 \mathrm{~s}$. For the $\mathrm{K}_{\mathrm{D}}$ determinations, the data were fit using a one-site binding model in the Origin ITC data analysis software.

\subsubsection{Circular Dichroism Studies}

Circular Dichroism spectra were obtained with an Aviv 202-01DS CD spectrometer (Aviv, Lakewood, N.J.) and processed using Origin 6.1. Experiments were performed by using a quartz cuvette with a $0.1 \mathrm{~cm}$ path length and $300 \mu \mathrm{l}$ volume. CD spectra were taken at $25^{\circ} \mathrm{C}$ in $50 \mathrm{mM}$ HEPES, $\mathrm{pH} 7.8,100 \mathrm{mM}$ potassium chloride, and $0.02 \%$ CHAPS. The stability tests of CCHD and CCHD/LD1 complex were carried by monitoring the $\mathrm{CD}$ signal at $222 \mathrm{~nm}$ while increasing the sample temperature from $25^{\circ} \mathrm{C}$ to $95^{\circ} \mathrm{C}$.

\subsubsection{NMR Spectroscopy and Backbone Assignment}

The p130cas ${ }^{738-874}$ used for structure determination was ${ }^{15} \mathrm{~N} /{ }^{13} \mathrm{C}$ double-labeled expressed and purified from construct p130Cas ${ }^{665-874}$ in $20 \mathrm{mM}$ HEPES, pH 7.5, $100 \mathrm{mM}$ potassium chloride and $5 \%(\mathrm{v} / \mathrm{v}) \mathrm{D}_{2} \mathrm{O}$. Final protein was concentrated down to $0.25 \mathrm{mM}$ 
addition of LD1 peptide at molar ratio of 1 to 5. The LD1 peptide was for forming complex to stabilize p130 cas $^{738-874}$. All NMR samples were approximately $500 \mu 1$, and the NMR spectra for structure determination were acquired at $25^{\circ} \mathrm{C}$ with the Bruker Avance $600 \mathrm{MHz}$ spectrometers and a Bruker Avance $800 \mathrm{MHz}$ spectrometer, all equipped with a cryoprobe. Multi-dimensional spectra HSQC, HNCA, HN(CO)CA, $\mathrm{CBCA}(\mathrm{CO}) \mathrm{NH}$, and $\mathrm{HNCACB}$ were acquired for backbone assignment. Spectra were processed by TopSpin 3.1 and analyzed by the software CARA.

\subsubsection{Crystallization, Structure Determination and Model Quality}

The protein $\mathrm{p} 130 \mathrm{Cas}^{738-874}$ was crystallized in the presence of LD1 peptide at 18 ${ }^{\circ} \mathrm{C}$ by sitting-drop batch crystallization. The $5 \mu \mathrm{l}$ drop contained $0.5 \mathrm{mM}$ protein and 2 $\mathrm{mM}$ peptide. The drop and $500 \mu \mathrm{l}$ well solution contained $20 \mathrm{mM}$ HEPES, $\mathrm{pH} 7.5$, and $50 \mathrm{mM} \mathrm{KCl}$. Crystals were cryopreserved by slowly increasing the glycerol concentration from $15 \%(\mathrm{v} / \mathrm{v})$ to $30 \%(\mathrm{v} / \mathrm{v})$ with $85 \%(\mathrm{v} / \mathrm{v})$ and $70 \%(\mathrm{v} / \mathrm{v})$ well solution, respectively. Native data were collected at SER-CAT BM beamline. Data were integrated and scaled to 2.0Å with HKL2000 (Otwinowski and Minor, 1997). Molecular replacement was performed using the previously published human p130Cas C-terminal domain crystal structure (PDB ID 3T6G, chain B) as the search model with Phaser (McCoy, 2007). The crystals belong to space group $C 2$, with three $1: 1$ protein/peptide complexes in the asymmetric unit (chains A/D, B/E, and C/F). Model building was performed using Coot (Emsley and Cowtan, 2004). Structure refinement, which included use of NCS restraints until the final stages, was performed using Phenix (Adams, 2010). Moreover, 5\% of the data was sequestered for the calculation of $R_{\text {free. }}$ Structure validation was performed with MolProbity (Chen, 2010). Ramachandran statistics show that $99.75 \%$ and $0.25 \%$ of the residues are in the preferred and allowed regions, respectively. Structural figures were generated with PyMOL (DeLano, 2002).

\subsection{Results}

\subsubsection{Defining the P130Cas CCHD}

The structure of p130Cas C-terminal has been revealed in the NSP3/Cas complex[230], showing that this region can form a four-helix bundle to interact with NSP3 and may function as a FAT-like domain. However, as a proposed FAT domain how CCHD interacts with the members of FAs remaining unclear. Based on the sequence analysis and secondary structure prediction, we confirmed that the $\mathrm{C}$-terminal region adopts a similar structure of the FAK FAT domain and may interact with other FA members as a FAT-like domain. In addition, we discovered a potential alpha helix region containing an Src binding site at the N-terminal of the four helix-bundle. It has been reported that some FAT-like domain may form a five alpha helix structure, which has an alpha helix addition to the four-helix bundle. In order to investigate and define the CCHD the p130Cas ${ }^{738-874}$ and p130Cas ${ }^{665-874}$ have been generated. The construct p130Cas ${ }^{665-874}$ 
contains the N-terminal potential helix, which is p130Cas ${ }^{665-737}$, and the FAT-like domain, which is p130Cas ${ }^{738-874}$. We specifically included a secondary thrombin cleavage site in between the p130Cas ${ }^{665-737}$ and p130 Cas $^{783-874}$ for allowing us to remove p130Cas ${ }^{665-737}$ when needed.

The attempt to express p130Cas ${ }^{738-874}$ in E.coli system failed, due to the stability and solubility. The four-helix bundle cannot form a stable structure and be maintained in solution. We then successfully expressed and purified p130Cas ${ }^{665-874}$ and confirmed that the p130Cas ${ }^{665-874}$ is folded by CD spectroscopy. The data from the CD spectrum clearly indicates p130Cas ${ }^{665-874}$ fold is dominated by helices, shown in Figure 4-2. Furthermore, we removed p130Cas ${ }^{665-737}$ from $\mathrm{p} 130 \mathrm{Cas}^{655-874}$ by thrombin cleavage to obtain the p130Cas ${ }^{665-737}$ mixing with p130Cas ${ }^{738-874}$. The p130 Cas ${ }^{738-874}$ cannot be further purified from the mixture indicating the p130Cas ${ }^{665-737}$ containing the potential helix may play a role to maintain the four-helix bundle structure. 2D HSQC spectroscopy has been carried out to study the sample p130Cas ${ }^{665-874}$ and the p130Cas ${ }^{738-874}$ mixture. The ${ }^{15} \mathrm{~N}-\mathrm{HSQC}$ spectrum of p130Cas ${ }^{665-874}$ is shown in Figure 4-3A, the widely dispersed amide peaks in the spectrum indicating well folded protein structure, and the center crowded region suggesting the protein contains a larger floppy region. The $15 \mathrm{~N}-\mathrm{HSQC}$ spectrum of p130Cas ${ }^{738-874}$ mixture is identical comparing with p130Cas ${ }^{665-874}$, shown in Figure 4-3B. This result is suggesting p130Cas665-737 may stabilize the four-helix bundle, which is p130Cas738-874, though cleaving p130Cas ${ }^{665-737}$ will not affect the integrity of the fourhelix bundle. Combining the previous study of NSP3/Cas complex structure and our NMR data, it is suggestive that CCHD consists of four alpha helixes from residue 738 to residue 874 and functions as a FAT-like domain, and in addition, a large floppy region containing the Src binding site can enhance the four-helix bundle formation.

\subsubsection{ITC Studies of the Paxillin LD Motifs Binding to P130Cas CCHD}

The CCHD is structurally alike the FAK FAT domain and function is proposed as a FAT-like domain that suggests the ability of interacting with focal adhesion members such as paxillin. Paxillin consists of the N-terminal LD motifs and C-terminal Lim domain. It has been recognized as an adaptor protein connecting the signal transduction in the Focal adhesions. Previous studies showed the LD motifs of paxillin can interact with multiple key player proteins in the Focal adhesions, such as FAK, GIT1, PYK2, and more. As in common, these proteins all are containing a FAT or FAT-like domain to interact with Paxillin LD motifs. We utilized p130Cas ${ }^{665-874}$ to investigate whether CCHD can localize p130Cas in FA through paxillin by ITC studies. Paxillin LD1, LD2, LD3, LD4, and LD5 have been synthesized and tested against purified p130Cas ${ }^{665-874}$ by ITC titrations. The result shows paxillin can interact with CCHD but only through the binding of the LD1 motif to p130Cas p130Cas ${ }^{665-874}$. Representative titration is shown in Figure 4-4. The experimental data of LD1 p130Cas ${ }^{665-874}$ best fit a one-site binding model and shows that LD1 peptide binds to $130 \mathrm{Cas}^{665-874}$ with a $\mathrm{K}_{\mathrm{D}}$ of approximate $4.2 \mu \mathrm{M}$. ITC results on LD2-LD5 titrating into p130Cas ${ }^{665-874}$ indicate these peptides have very weak or no binding affinity to $\mathrm{p} 130 \mathrm{Cas}^{665-874}$. This data suggests paxillin interacting $\mathrm{p} 130 \mathrm{Cas}$ through the association of the LD1 motif with CCHD. 


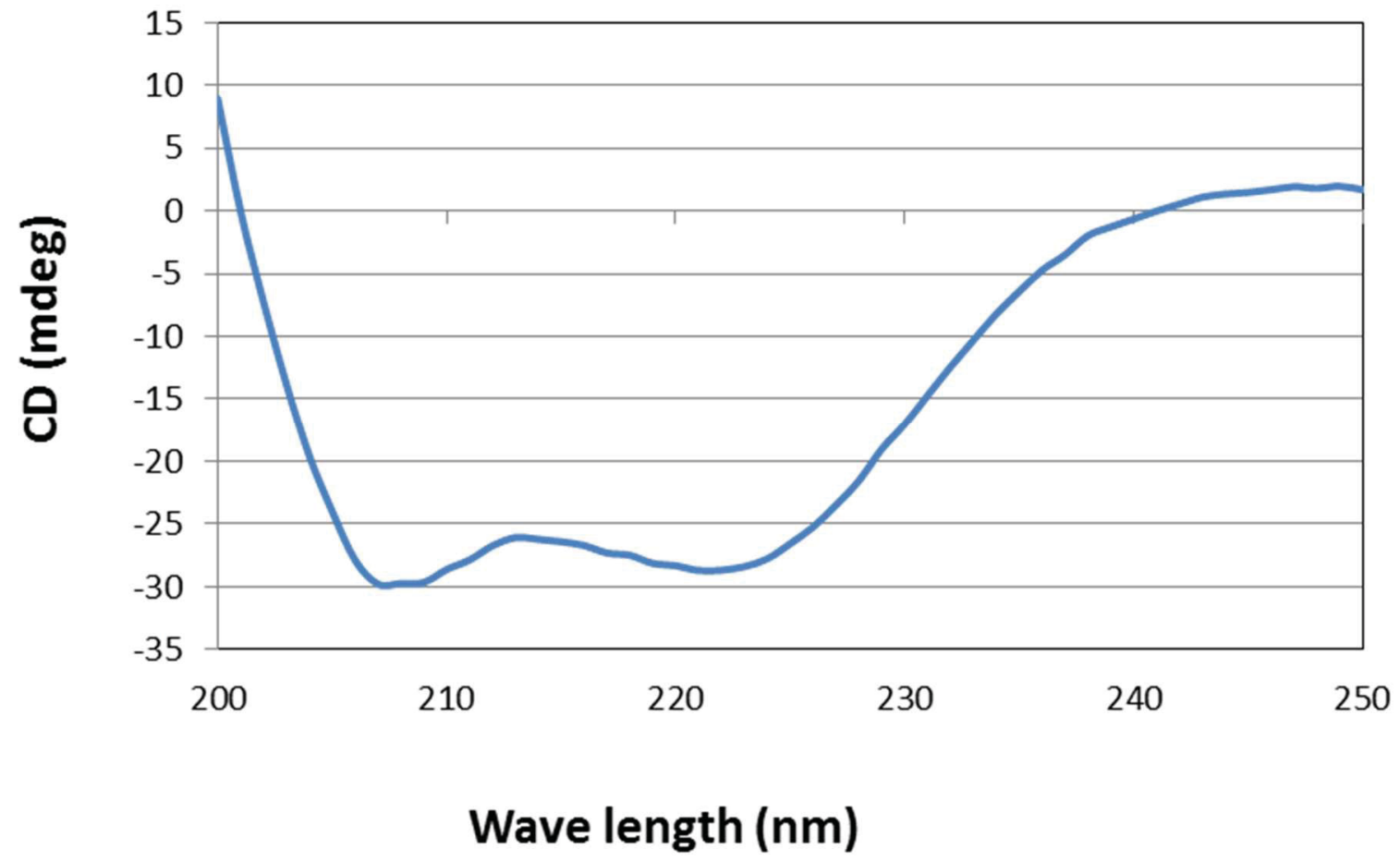

Figure 4-2. CD spectra of the p130Cas ${ }^{665-874}$.

The CD spectra showing the signature peaks of alpha helical structure at $210 \mathrm{~nm}$ and 222 $\mathrm{nm}$ are indicating that $\mathrm{p} 130 \mathrm{Cas}^{665-874}$ is folded and dominated by alpha helixes. 
A

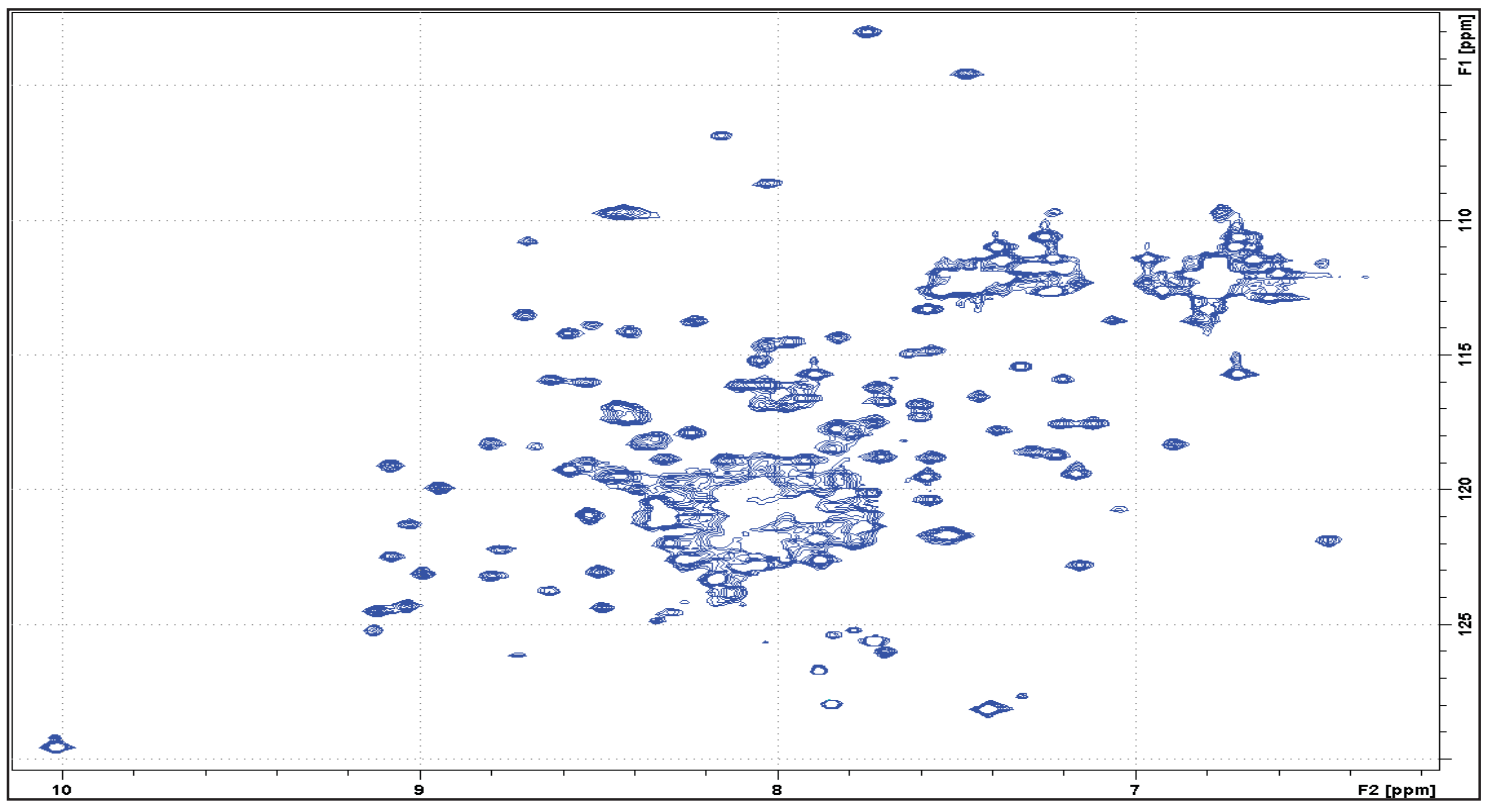

B

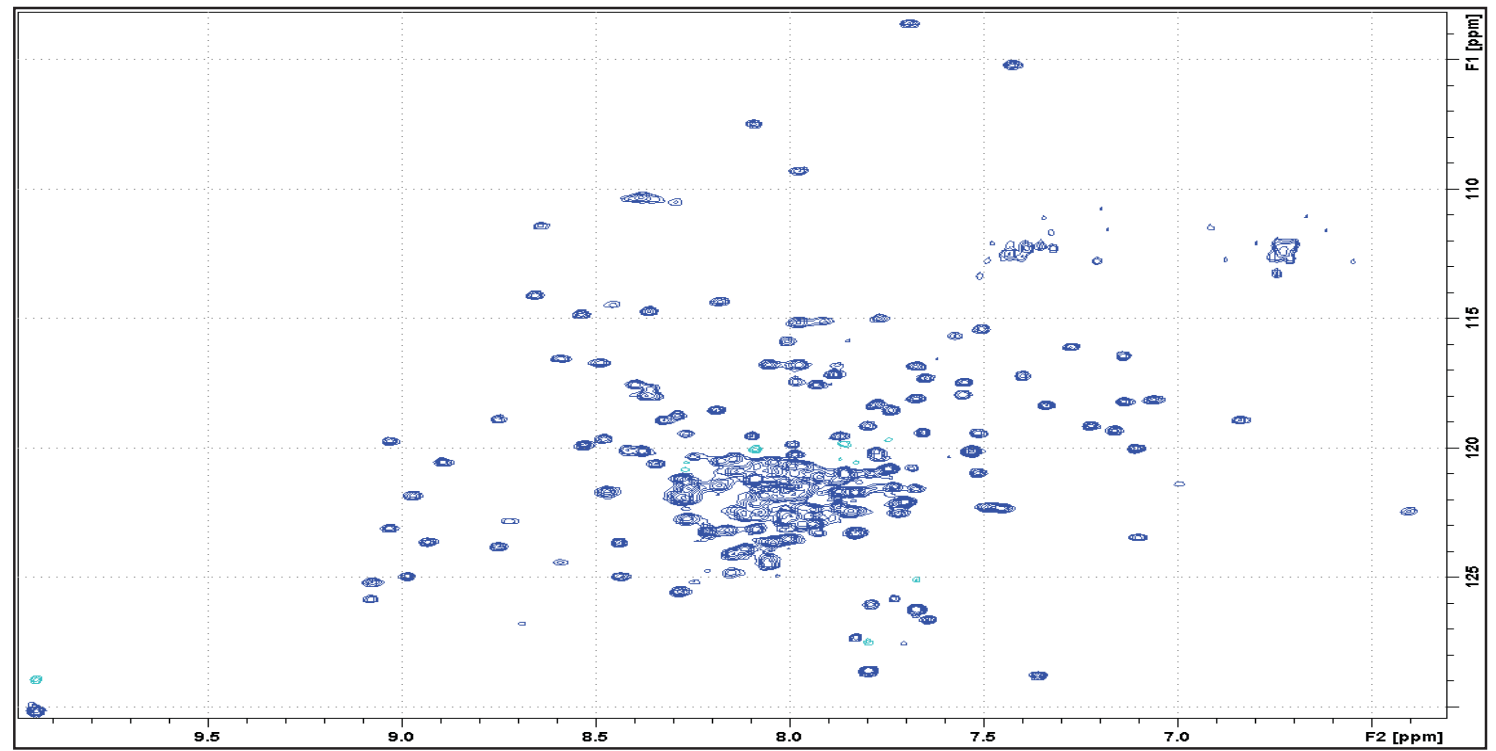

Figure 4-3. ${ }^{15} \mathrm{~N}-\mathrm{HSQC}$ spectra of p130Cas CCHD.

(A) The ${ }^{15} \mathrm{~N}-\mathrm{HSQC}$ spectrum of the p130Cas ${ }^{665-874}$. The well-dispersed peaks are suggesting the purified protein is folded, and center crowed peaks indicates the protein contains a large floppy region. (B) The ${ }^{15} \mathrm{~N}-\mathrm{HSQC}$ spectrum of $\mathrm{p} 130 \mathrm{Cas}{ }^{665-737}$ and p130 Cas ${ }^{738-874}$ mixture is identical to the spectrum of p130Cas ${ }^{665-874}$. 


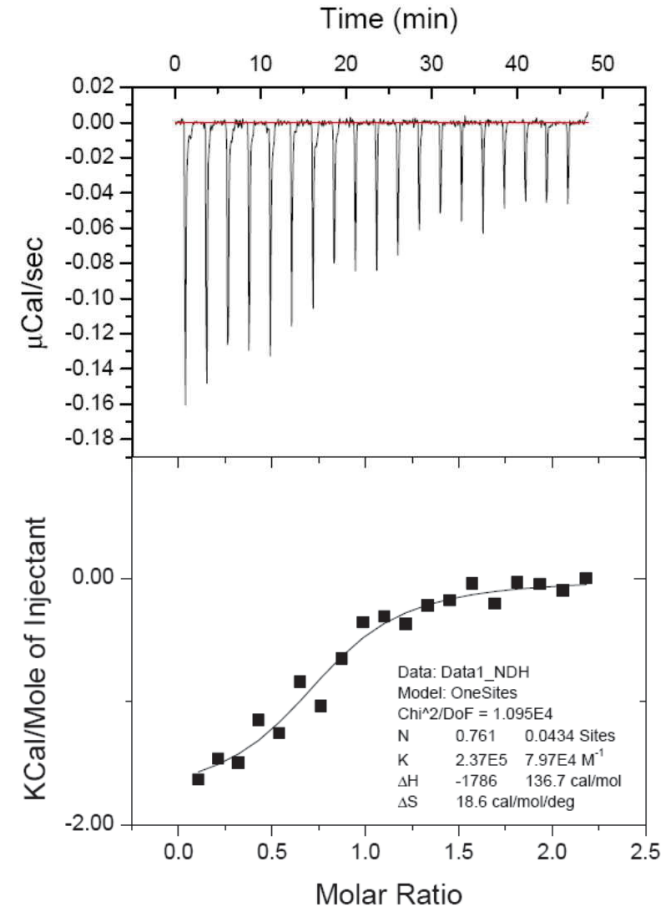

CAS w/ LD1

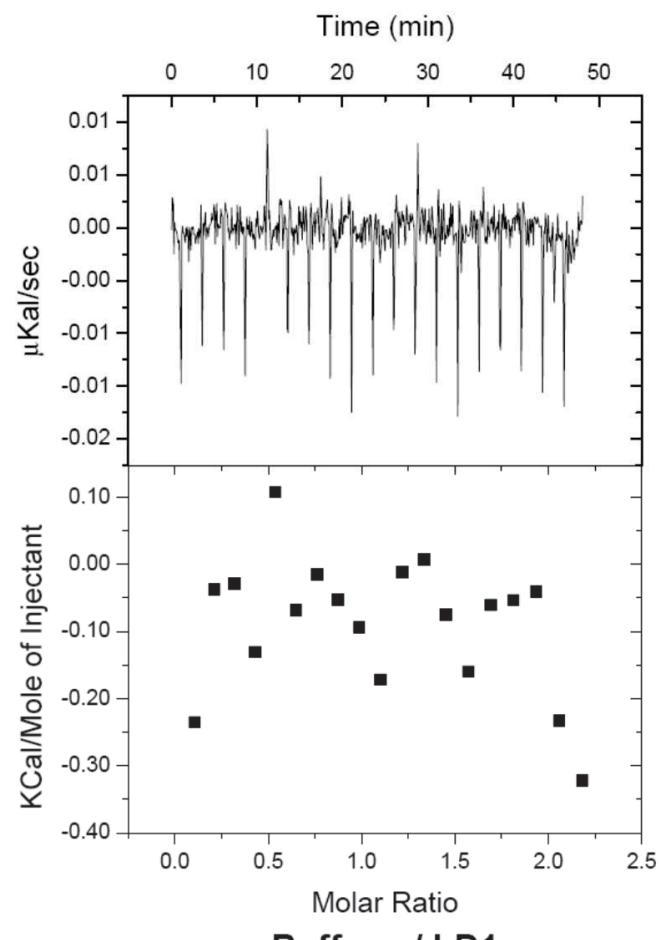

Buffer w/ LD1

Figure 4-4. ITC titrations show LD1 of paxillin can bind to CCHD of p130Cas. ITC study of paxillin/p130Cas interaction: (A) Synthetic LD1 peptide has been titrated into purified p130Cas ${ }^{665-874}$ showing a binding affinity of approximate $4.2 \mu \mathrm{M}$ in $\mathrm{K}_{\mathrm{D}}$. (B) The blank LD1 to buffer titration is shown as the control experiment. 


\subsubsection{P130Cas CCHD Stabilized in Complex with Paxillin LD1}

In our previous protein purification we were able to express and purify the p130Cas ${ }^{665-874}$ and remove the unwanted $p 130$ Cas $^{665-737}$ by thrombin cleavage to obtain the p130 Cas ${ }^{738-874}$, which contains the pure CCHD, in mixture with p130 Cas ${ }^{665-737}$. However, the effort to further isolate the p130 Cas ${ }^{738-874}$ from the mixture solution has failed. Therefore, the initial aim to purify a stable CCHD for NMR or crystallography study seems impossible. By identifying the binding partner we were able to replace the p130 Cas ${ }^{665-737}$ from solution to stabilize CCHD by LD1 instead. We then included LD1 in the entire purification procedure and this results in largely increasing the yield of the stable and soluble CCHD (p130 Cas ${ }^{738-874}$ ) in a complex form with LD1. These data indicate the LD1 can stabilize CCHD in solution. To confirm this we performed a CD melting study to compare the stability of CCHD with or without LD1 peptide, shown in Figure 4-5. Indeed, the data agree with our hypothesis.

Furthermore, we tested the quality of the stabilized CCHD on a molecular level. As the NMR spectroscopy technique has been widely used to evaluate the quality of protein samples, the acquisition of ${ }^{15} \mathrm{~N}-\mathrm{HSQC}$ spectra has been performed. A good quality protein sample that can be used for NMR structural study must display a ${ }^{15} \mathrm{~N}$ HSQC spectrum with well-dispersed, uniformed peaks shape, and consistent peak numbers of target protein characterizing a well folded protein. The p130Cas ${ }^{738-874} / \mathrm{LD} 1$ complex showed the improvement in $15 \mathrm{~N}-\mathrm{HSQC}$ spectra comparing with p130Cas ${ }^{738-874}$ mixture, shows in Figure 4-6. From the spectrum we have observed that most peaks are uniform in shape and evenly dispersed, which indicates the correct folding of p130Cas ${ }^{738-}$ ${ }^{874} / \mathrm{LD} 1$. The N-terminal region to the CCHD in p130Cas ${ }^{665-874}$ stabilized CCHD and facilitated the expression of soluble protein, however with the binding of LD1, removing the p130Cas ${ }^{665-737}$ is necessary for obtaining the clear spectra for studying the CCHD/LD1complex structure. To serve this purpose, the construct p130Cas ${ }^{665-874}$ was cloned for obtaining a better quality sample to investigate the complex structure information. We designed and introduced a thrombin cleavage site in between p130 Cas ${ }^{665-737}$ and p130 ${ }^{2}{ }^{738-874}$. With this approach, we utilized the N-terminal potential helix helical region $\mathrm{p} 130 \mathrm{Cas}^{665-737}$ to facilitate protein production, and then we removed it after CCHD binding with LD1 by thrombin digestion.

\subsubsection{NMR Study of the CCHD}

${ }^{15} \mathrm{~N}$ and ${ }^{13} \mathrm{C}$ double-labeled p130Cas ${ }^{665-874}$ has been expressed and purified along with unlabeled LD1 peptide. The final NMR sample is cleaved p130Cas ${ }^{736-874}$ in a concentration of about $0.2 \mathrm{mM}$, with the LD1 in molar ration 1:5. 2D ${ }^{15} \mathrm{~N}-\mathrm{HSQC}$ spectrum; and 3D ${ }^{15} \mathrm{~N}-{ }^{13} \mathrm{C}-\mathrm{HNCA},{ }^{15} \mathrm{~N}-{ }^{13} \mathrm{C}-\mathrm{HNCACB}$, and ${ }^{15} \mathrm{~N}-{ }^{13} \mathrm{C}-\mathrm{CBCA}(\mathrm{CO}) \mathrm{NH}$ were obtained for backbone assignment. In ${ }^{15} \mathrm{~N}-\mathrm{HSQC}$ experiment the correlation between each amide proton and the connected amide nitrogen atom is measured. Except for proline, each amino acid in the protein has one amide pair in the backbone, which can be detected in the HSQC experiment. In addition, the amide side chains of the glutamine and the asparagine residues are also observed. From the ${ }^{15} \mathrm{~N}-\mathrm{HSQC}$ spectrum, the consistent 


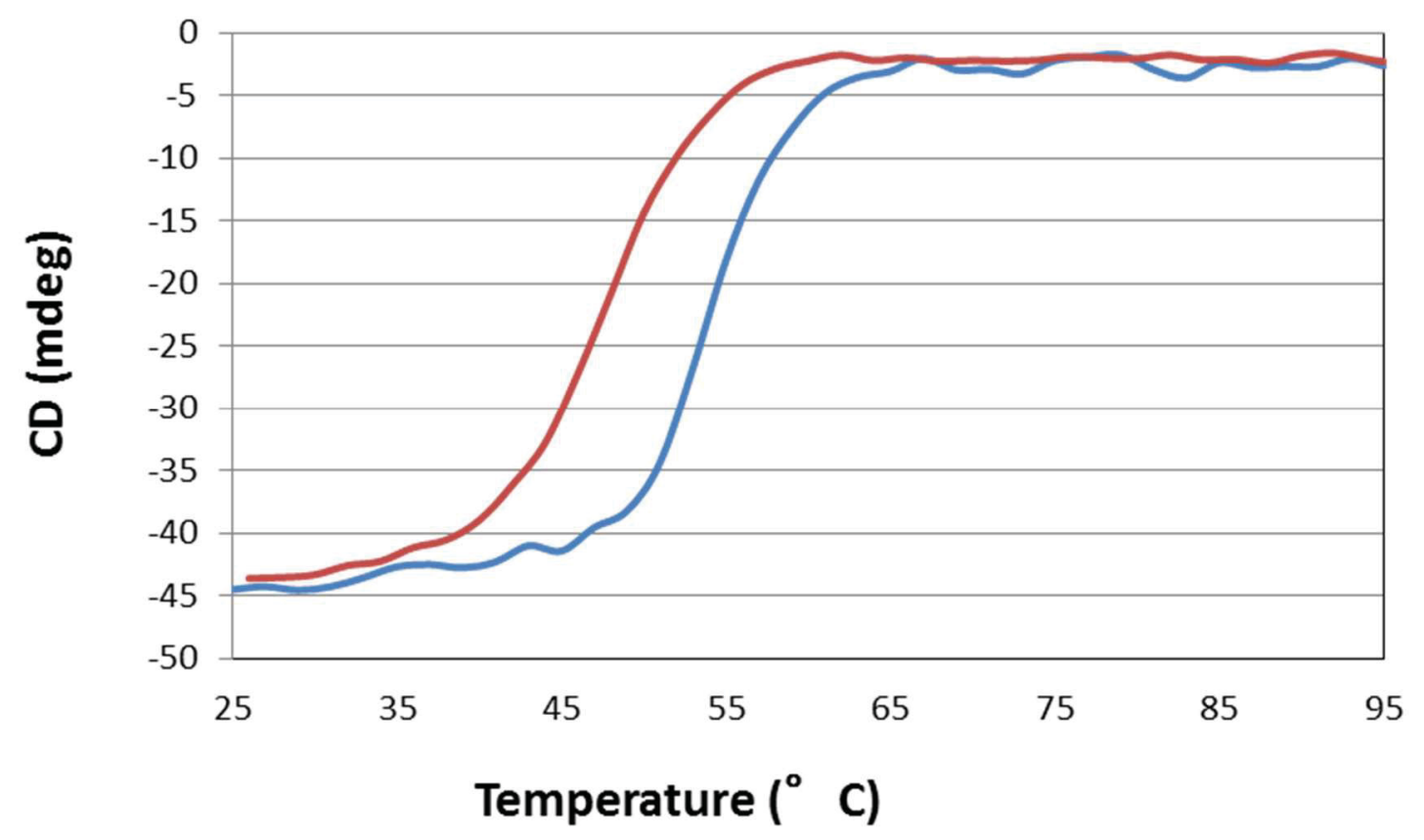

Figure 4-5. CD spectra of p130Cas ${ }^{665-874}$ with or without LD1 peptide.

Show in red color is the CD melting curve of p130Cas ${ }^{665-874}$ and in blue is the melting curve of p130 Cas ${ }^{738-874}$ with LD1. In this experiment we specifically monitored the wavelength of the signature alpha helix peak in CD spectra, which is $222 \mathrm{~nm}$. We heated up the sample from $25^{\circ} \mathrm{C}$ to $95^{\circ} \mathrm{C}$ for performing a melting test. From the data we see the CCHD alone melts earlier than CCHD complex with LD1. This data is suggesting that in the complex with LD1 the stability of CCHD has been enhanced, which agrees with our hypothesis. 


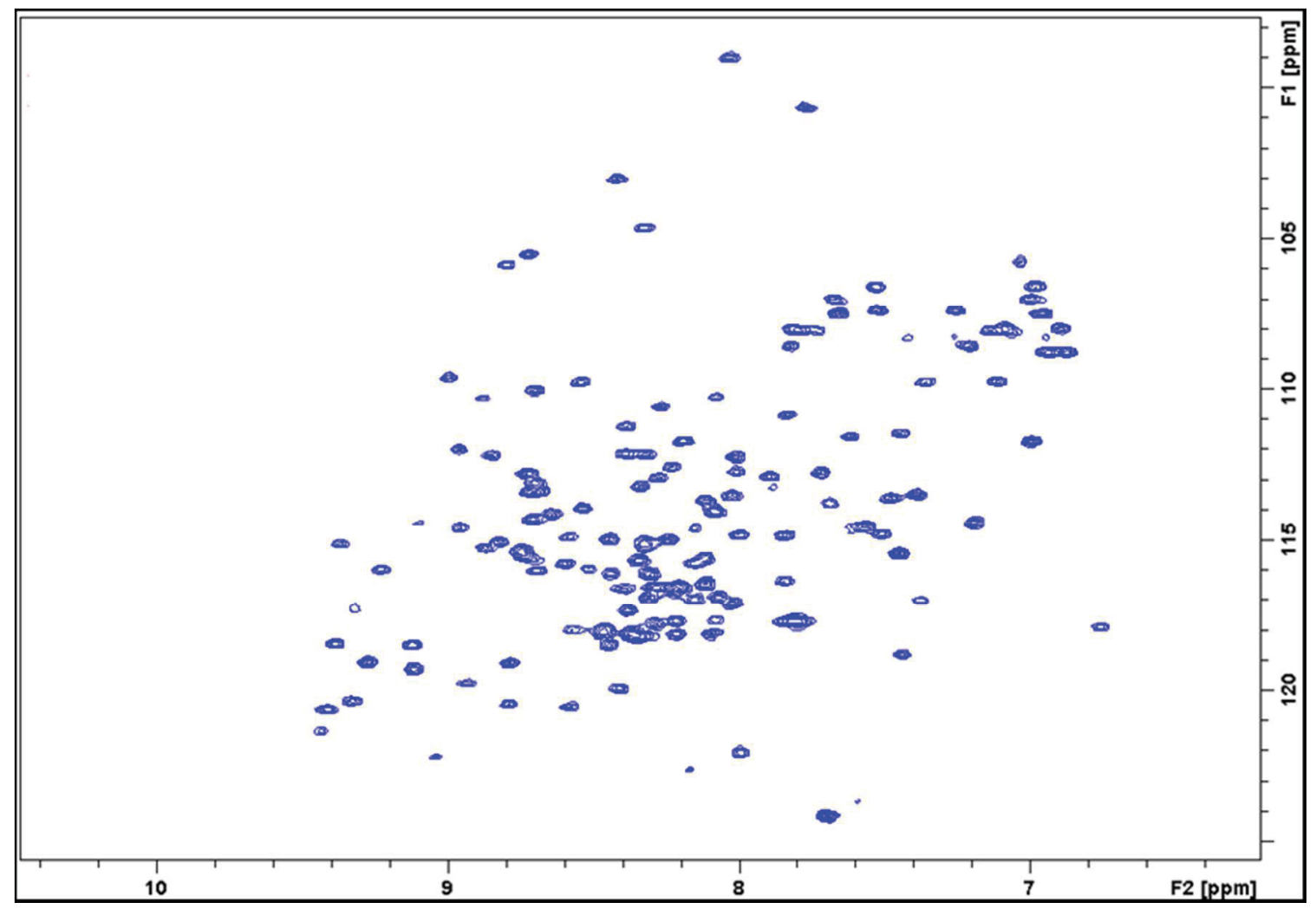

Figure 4-6. ${ }^{15} \mathrm{~N}-\mathrm{HSQC}$ spectra of p130Cas CCHD complex with paxillin LD1. The ${ }^{15} \mathrm{~N}-\mathrm{HSQC}$ spectrum of the ${ }^{15} \mathrm{~N}$ labeled p130Cas ${ }^{738-874}$ complex with unlabeled LD1 peptide. Peaks show in the spectra representing the amides of p130Cas ${ }^{738-874}$ and peaks uniform in shape and evenly dispersed, which indicates the well folded structure of p130Cas ${ }^{738-874 / L D 1 . ~}$ 
peak number with residue number of CCHD and well dispersed peaks suggests that the CCHD is folded. The backbone assignments were performed using the triple resonance approach for the double-labeled CCHD sample.

\subsubsection{Crystal Structure of the CCHD Complex with LD1}

We solved the crystal structure of LD1 bound to CCHD at a best resolution of 2.0 $\AA$ (crystal structure solved by Dr. Darcie miller), the crystal table is shown in Table 4-1. The asymmetric unit of the crystal contains three LD1/CCHD complexes, which are clearly defined by electron density, shown in Figure 4-7. In agreement with our ITC characterization, the crystal structure shows that the CCHD protein and LD1 peptide form a molar ratio 1:1 arrangement. The p130Cas CCHD forms a compact rod-like domain structure with well-defined terminuses. The proximity of both terminuses is a feature commonly appearing in functional independent domains. The CCHD structure forms a four-helix-bundle with a right-handed up and down topology, with approximate $50 \AA$ in height. The four helices form an antiparallel bundle, and the hydrophobic side chains from all helices interlace forming a hydrophobic core that dramatically stabilizes the bundle. Of the four helices in the CCHD structure, helix 2 (28 residues) is slightly shorter than helix 1 ( 32 residues), helix 3 ( 31 residues), and helix 4 (30 residues). The p130Cas CCHD has 4 prolines, that all locate in the loop region in between helices. The rigid backbone and hydrophobic side chain of proline makes the loops less flexible, that allowing us to observe extensive electron density. The structure of CCHD complex with LD1 further confirms that the p130Cas CCHD is a FAT-like domain, both functional and structural, which adopts a well-defined four-helix bundle. Extensive hydrophobic interactions in the core of the domain suggest a stable arrangement of the helices. However, the N-terminal helix (residue 665-737) may important for the initial four-helix bundle structure formation. By comparing the sequence of the p130Cas CCHD with those others FAT and FAT-like domains, which adopt the similar four-helix bundle structure, the FAT domain of FAK, the Protein Binding Domain (PDB) of GIT1, and the FAT domain of PYK2, that the hydrophobic residues in the core regions are well conserved among all these sequences. In p130Cas CCHD structure, there are total 17 leucines and 14 of them facing toward to the central hydrophobic core providing strong hydrophobic interaction. For the best representative residues, the Leu 758 from helix 1, Leu 792 from helix 2, Leu 819 from helix 3, and Leu 853 from helix 4, form a leucine cluster that dramatically stabilize the central hydrophobic core, show in Figure 4-8.

Generally, FAT and FAT-like domains function as adaptor domains in focal adhesion signaling, interacting with binding partners by recruiting helical motifs to surface grooves between their antiparallel helices[231]. In our crystal structure we observe the LD1 motif of paxillin can interact with CCHD of p130Cas through hydrophobic interaction. 
Table 4-1. Data collection and refinement statistics.

\begin{tabular}{|c|c|}
\hline Name & p130Cas ${ }^{738-894} / \mathrm{LD} 1$ \\
\hline \multicolumn{2}{|l|}{ Data collection } \\
\hline Space group & $C 2$ \\
\hline \multicolumn{2}{|l|}{ Cell dimensions } \\
\hline$a, b, c(\AA)$ & $84.3,37.7,152.5$ \\
\hline$\alpha, \beta, \gamma\left(^{\circ}\right)$ & $90.0,100.9,90.0$ \\
\hline Resolution $(\AA)$ & $2.0(2.07-2.00)^{\mathrm{a}}$ \\
\hline$R_{\mathrm{sym}}(\%)$ & $6.0(37.6)$ \\
\hline$I / \sigma I$ & $30.3(2.5)$ \\
\hline Completeness (\%) & $97.3(77.1)$ \\
\hline Redundancy & $5.7(3.3)$ \\
\hline \multicolumn{2}{|l|}{ Refinement } \\
\hline Resolution $(\AA)$ & $30.0-2.0$ \\
\hline No. reflections & 31,396 \\
\hline$R_{\text {work }} / R_{\text {free }}$ & $19.6 / 23.7$ \\
\hline \multicolumn{2}{|l|}{ No. atoms } \\
\hline Protein & 2,877 \\
\hline Peptide & 247 \\
\hline Water & 181 \\
\hline \multicolumn{2}{|l|}{$B$-factors } \\
\hline Protein & 44.6 \\
\hline Water & 47.3 \\
\hline \multicolumn{2}{|l|}{ R.m.s. deviations } \\
\hline Bond lengths $(\AA)$ & 0.007 \\
\hline Bond angles $\left(^{\circ}\right)$ & 0.009 \\
\hline
\end{tabular}

Dataset was collected from a single crystal.

${ }^{a}$ Values in parenthesis are for the highest resolution shell. 


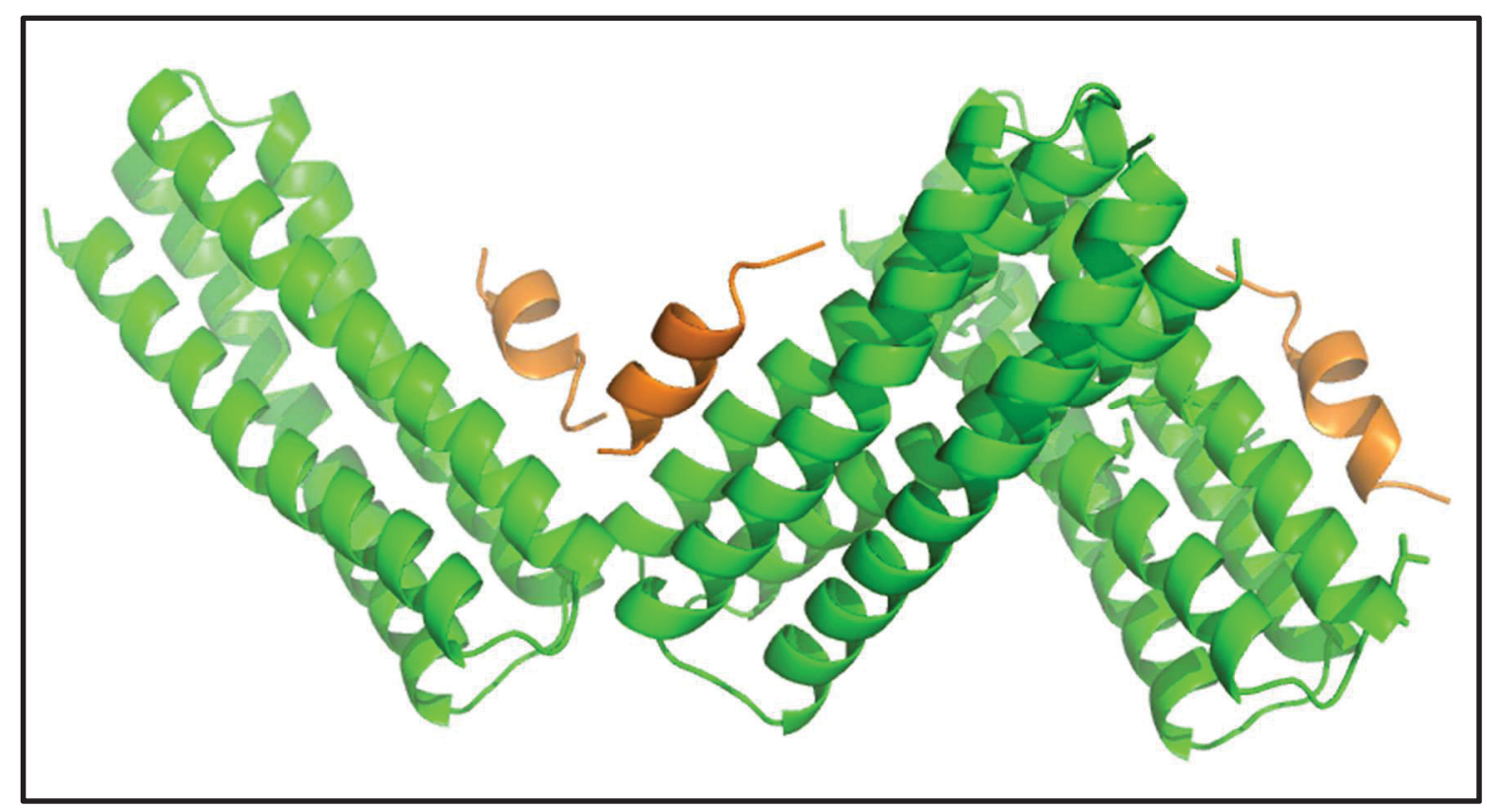

Figure 4-7. Crystal structure of p130Cas CCHD complex with paxillin LD1. Each of asymmetric unite of the CCHD/LD1 complex crystal contains three protein complexes, which are clearly defined by electron density. The crystal structure shows that the CCHD protein and LD1 peptide form a molar ratio 1:1 arrangement. 


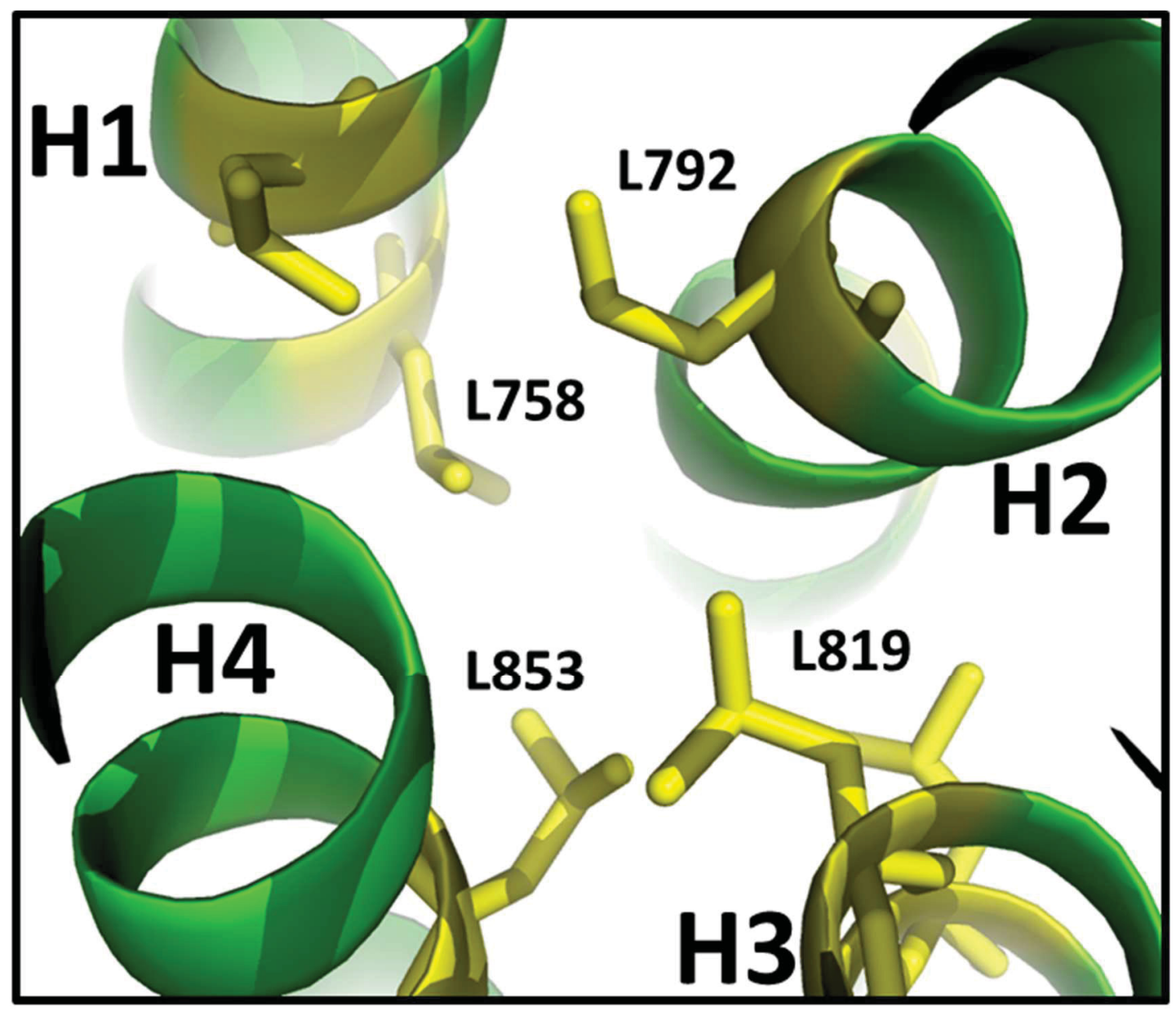

Figure 4-8. 3D structure of the CCHD hydrophobic core.

The key residues to form the hydrophobic core of CCHD are colored in yellow. These residues are extremely conserved from species that play an important role in maintaining the four-helix bundle structure of FAT and FAT-like domains. 


\subsubsection{LD1 Motif of Paxillin Binds to CCHD at the H2/H3 Surface}

The binding between the p130Cas CCHD and paxillin is essential to localize the FAK-p130Cas complex to the focal adhesion [145]. Although the function of CCHD targeting focal adhesion had been proposed, the detailed mechanism remained unknown. Therefore, our complex structure provides a tool to address this question at the molecular level. It has been well known that the Paxillin functions as a adaptor protein, and its LD motifs create five binding surfaces for the recruitment of FA proteins [232]. Its also been reported LD1 motif can interact with actopaxin [233], vinculin [115], and the integrinlinked kinase [231]. LD2 mediates the binding to vinculin, FAK and PYK2 [234]. LD4 binds to actopaxin, FAK and PYK2 [233] [234]. Thus far, no binding partners of LD3 and LD5 have been reported.

In our study, the complex structure shows the CCHD interacts with paxillin mediated by the LD1 motif. The binding site of LD1 locates to the central region of the $\mathrm{H} 2$ and $\mathrm{H} 3$ solvent-exposed surface, which clearly shows in electron density map. LD1 binds to the CCHD H2/H3 surface mainly through the hydrophobic interaction mediated by the hydrophobic side chain of leucine residues inserting into the hydrophobic grooves in between H2/H3 of CCHD. Furthermore, the charged residues Asp 2 and Asp 10 forming the salt bridge with Arg 828 and Lys 835 from $\mathrm{H} 3$ of CCHD respectively, providing a specific anchor point for LD1. The Met 1 of the LD1, which is the very first residue of the paxillin, interact with Phe 798 from $\mathrm{H} 2$ through hydrophobic interactions. This provides a docking space for the N-terminal of LD1. These key residues have been verified by the simulated annealing omit map shown in Figure 4-9.

\subsubsection{Comparison of P130Cas FAK with the FAK FAT Domain}

Forming four amphipathic helices, the p130Cas CCHD buries most of its hydrophobic side chains in the bundle core. There is only one major hydrophobic patch on the surface. As shown in Figure 4-10, side chains from $\mathrm{H} 2$ and $\mathrm{H} 3$ form two positivecharged ridges sandwiching an elongated hydrophobic groove, which covers about two thirds of the four-helix bundle length. To the center of the H2/H3 surface, positively charged Lys 788, Arg 828, Lys 835 enclose a well-defined hydrophobic pocket composed with hydrophobic residues at the bottom. These residues are conserved in all organisms for which sequences are available. Among them, Lys788, Arg 828, Lys 835 are also conserved with the FAK FAT domain. This surface makes an ideal binding site for paxillin LD motifs, as confirmed by electron densities.

When the p130Cas CCHD was compared with the FAT domain of FAK, the latter has two major hydrophobic grooves located at both the $\mathrm{H} 1 / \mathrm{H} 4$ and the $\mathrm{H} 2 / \mathrm{H} 3$ faces, which correspond to the LD2 and LD4 motif binding sites, respectively. Both grooves have a surface setting similar to that of the H2/H3 site of the CCHD: a hydrophobic groove with surrounding positive-charged ridges. Superimposition of the structures of the PBD and the FAT domain revealed that the charge residues were in the same position, which is another indication of functional similarity arising from structural similarity. 


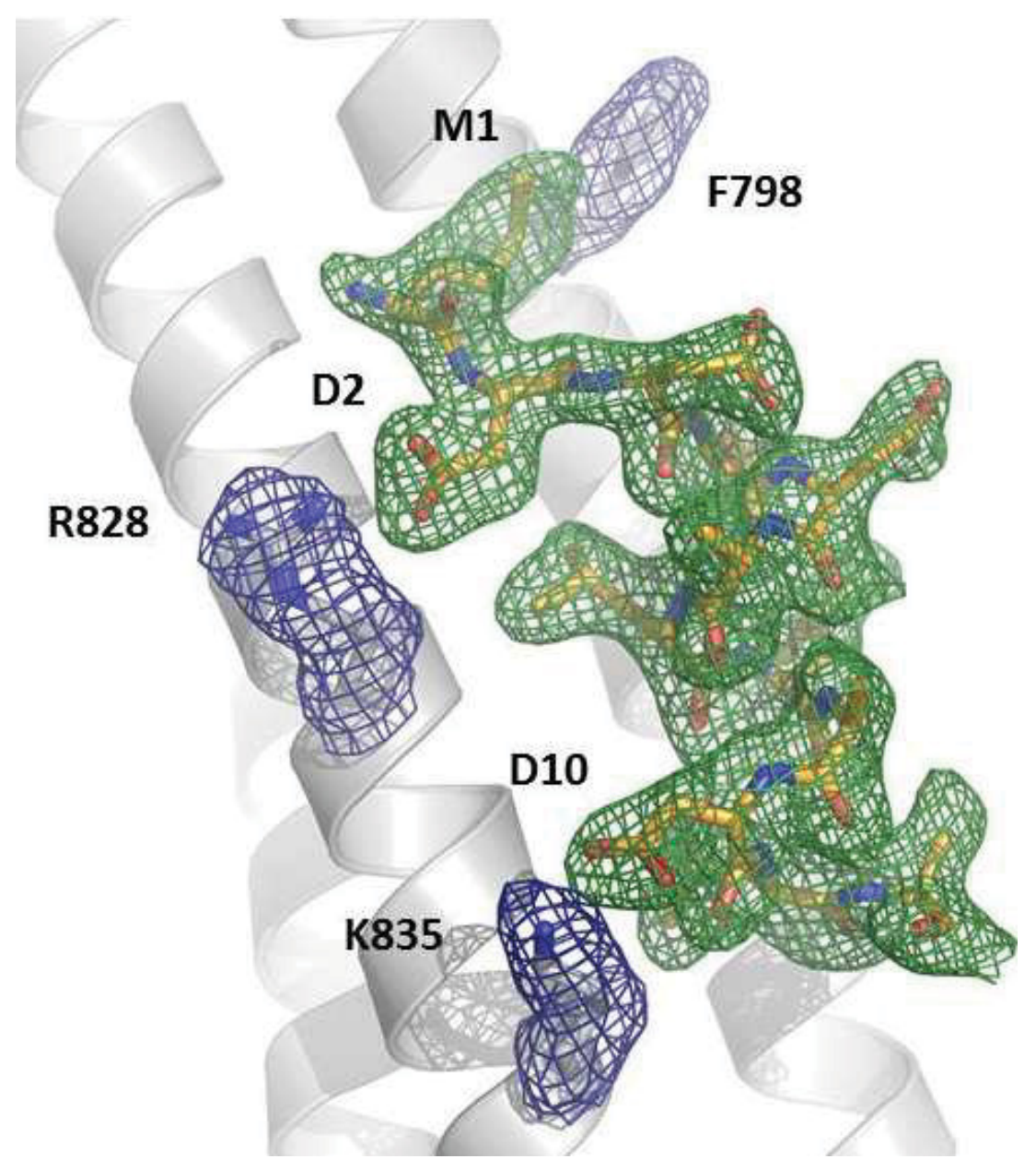

Figure 4-9. Representative electron density for p130Cas ${ }^{738-874} / \mathrm{LD1}$.

Fo-Fc simulated annealing omit maps contoured at $3 \sigma$ are shown for a few key p130Cas ${ }^{738-874}$ residues (blue density, grey sticks) and LD1 peptide (green density, gold sticks). The protein/peptide complex corresponding to chains A/D is shown. 

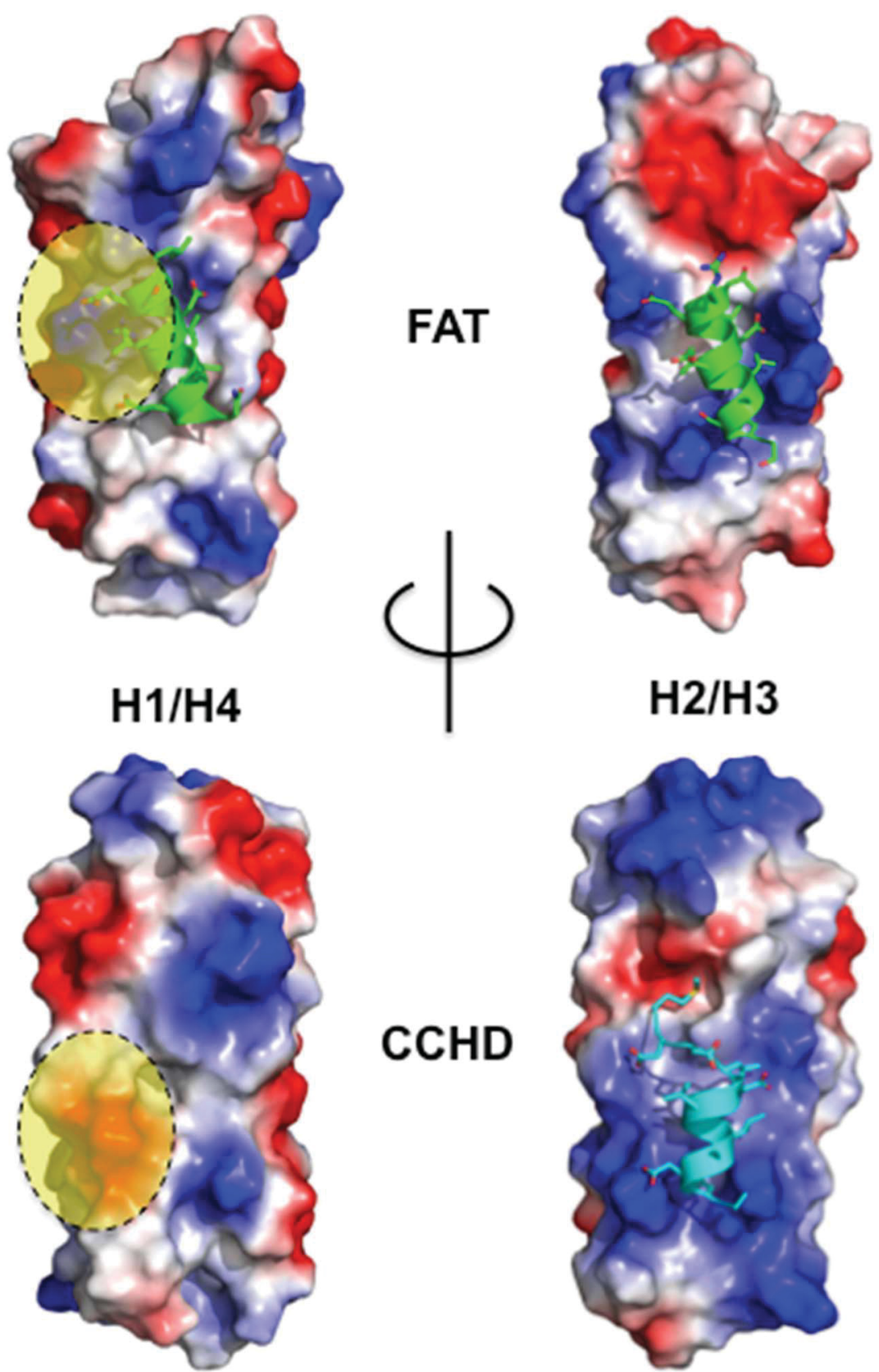

$\mathrm{H} 2 / \mathrm{H} 3$

\section{CCHD}

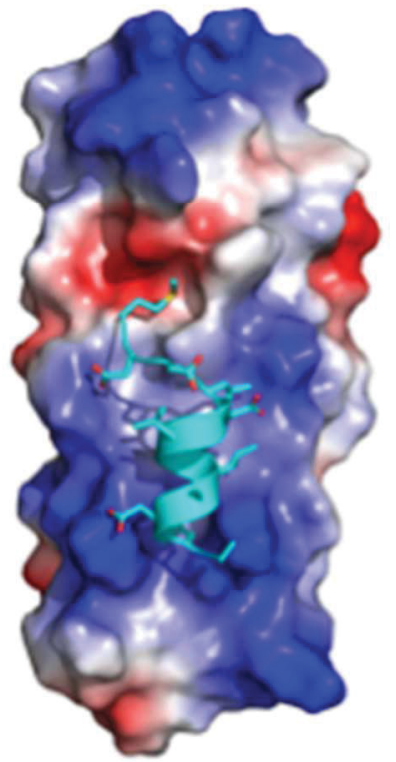

Figure 4-10. Elucidation of differences between the H1/H4 site on FAT and CCHD. Both CCHD and FAT are composed of two hydrophobic patches by helices H1/H4 (H1/H4 site) and helices $\mathrm{H} 2 / \mathrm{H} 3$ (H2/H3 site) respectively. The charged residues surrounding the hydrophobic patches form a charged sidewall creating the binding pocket. Unlike FAT on the $\mathrm{H} 1 / \mathrm{H} 4$ site of CCHD has a reversed charge, which is colored yellow in the figure, that results in the $\mathrm{H} 1 / \mathrm{H} 4$ site of CCHD no longer favorable for binding of LD motifs. 
The FAT surface has two hydrophobic surfaces that serve as LD motif binding sites, but the p130Cas CCHD has only one such surface, shown in Figure 4-10. In the FAT domain, helices $\mathrm{H} 1$ and $\mathrm{H} 4$ form a second hydrophobic surface, and at the center of the FAT H1/H4 site, the positive charge side wall of the hydrophobic pocket is composed of K933 and K1032 on H1 and H4, respectively. However, the equivalent position on p130Cas CCHD is occupied by negative charge Asp 766 that effectively close the pocket. Moreover, on the CCHD H2 and H3 binding surface, the Asp 781, Phe 798, and His 794 composed a small hydrophobic pocket that the N-terminal residue M1 of LD1 can perfectly fit in by hydrophobic interaction. This may provide binding specificity and explain why only LD1 of Paxillin can bind to CCHD.

\subsection{Discussion}

The crystal structure of the p130Cas CCHD is a stable four-helix bundle, which is similar to the structures of the FAT domain of FAK and the PYK2-FAT domain. The CCHD forms an up-and-down antiparallel helix bundle. CCHD of p130Cas, the FAT domain of FAK and the PYK2 FAT domain all share a well-conserved hydrophobic core, but their surface residues exhibit more diversity. This explains the very similar overall folding of the three domains as well as their distinct binding specificity. The FAK FAT domain interacts with the LD2 and LD4 motifs of paxillin while p130Cas binds only to LD1 motif of Paxillin. The binding surface composed by $\mathrm{H} 2 / \mathrm{H} 3$ of CCHD is very similar to FAT of FAK. However, there are distinct differences between the two domains. First, in solution, the FAT domain forms a dimer by swapping the H1 between the two promoters, whereas the CCHD is a well-folded monomer. Second, the LD2 and LD4 motifs of paxillin interact with the FAT domain simultaneously at two opposite faces of the four-helix bundle, whereas the only LD1 motifs of paxillin bind to the PBD at only one site, the $\mathrm{H} 2$ and $\mathrm{H} 3$ site. The crystal structure suggests the $\mathrm{H} 1 / \mathrm{H} 4$ site of p130Cas CCHD is closed by switching the positive charge residue to negative. These functional differences between the FAT domain and the CCHD are rooted in the difference of their sequences.

That the previously reported CCHD is equally as important as SH3 for p130Cas focal adhesion localization was confirmed in our studies. Furthermore, we addressed the mechanism of how the CCHD domain facilitates the p130Cas focal adhesion targeting at the molecular level. From our data, Paxillin, which is the focal adhesion marker, plays an important role in recruiting p130Cas into the focal adhesion complex. The LD1 motif of Paxillin binds to the $\mathrm{H} 2$ and $\mathrm{H} 3$ site of the CCHD, unlike the complex of Paxillin with the FAT domain of FAK, which interacts through LD2 and LD4 with $\mathrm{H} 1 / \mathrm{H} 4$ and $\mathrm{H} 2 / \mathrm{H} 3$ respectively. This variation may also explain the selectivity of LD motifs interacting with different Focal Adhesion members.In this study, we showed the similarity between the FAT domain of FAK and the CCHD of p130Cas, which both adopts four-helix bundle structure and the helices from the helix bundle compose a larger hydrophobic groove for binding of Paxillin LD motifs; and the difference from CCHD to FAT domain, with CCHD only containing one LD binding site composed by $\mathrm{H} 2 / \mathrm{H} 3$, while FAT has both $\mathrm{H} 2 / \mathrm{H} 3$ and $\mathrm{H} 1 / \mathrm{H} 4$ binding to LD4 and LD1 respectively. These data suggests the Paxillin 
may be connecting both FAT domains of FAK and CCHD of p130Cas simultaneously, through the binding of LD2 and LD4 with FAT, and the LD1 with CCHD.

The FAT domain of FAK binds to Paxillin by interacting with both the Paxillin LD2 and LD4 motifs simultaneously using its $\mathrm{H} 1 / \mathrm{H} 4$ and $\mathrm{H} 2 / \mathrm{H} 3$ sites. With two binding sites, FAT should have a much stronger binding affinity with Paxillin than the p130Cas CCHD since the CCHD has only one LD binding site, the $\mathrm{H} 2 / \mathrm{H} 3$ site. The relatively weak interaction may not sufficient for p130Cas targeting Focal adhesion. This data agree with previous studies, in which both the SH3 domain and the CCHD are necessary for p130Cas Focal adhesion localization. This mechanism could eliminate the interaction between p130Cas and FAK outside of the focal adhesion complex, since the FAK also plays an important role in cell cytoplasm.

Previous studies showed as a working model, focal adhesion members, such as FAK, PYK2, and GIT1 compete for Paxillin binding, and, in turn, these proteins compete for focal adhesion localization. When Paxillin Ser 272 is dephosphorylated, Paxillin prefers binding to FAK, because FAK binds both the Paxillin LD2 and LD4 motifs cooperatively with a higher affinity. Once Ser 272 is phosphorylated, the affinity between LD4 and FAK is weakened, causing the disassociation of FAK-LD4. However, the phosphorylation of S272 in LD4 does not affect the interaction between Paxillin with other focal adhesion members, such as GIT1. Therefore, Paxillin Ser 272 phosphorylation shifts the balance of the Paxillin interaction with FAK versus GIT1 in favor of GIT1 and causes an increase in the Paxillin-GIT1 complex. This balance shift may promote regulated FA assembly and the disassembly cycle, further affected p130Cas-FAK interaction. 


\section{CHAPTER 5. IMPLICATIONS AND FUTRE DIRECTIONS}

\subsection{Helical Constrained Peptide Targets FAK FAs Localization as an Inhibitor}

The first half of the dissertation presents a study showing helically constrained LD4-L6A peptide targets the FAK/paxillin complex as a peptidomimetic inhibitor. Numerous studies have indicated that elevated FAK expression and enhanced activity is associated with malignancy of various tumor cells, including neuroblastoma, melanoma, and colon cancer [61]. In tumor cells, decreasing of FAK expression induces cell detachement and apoptosis $[62,63]$. Studies have reveled that overexpression of wildtype FAK in tumor cells induces apoptosis and decreases cell proliferation, while knocking-down FAK by shRNA increases cell proliferation [63-65]. Given the role of FAK in tumorgenesis and malignancy, it could be a promising target for cancer therapy. TAE226, a small molecule inhibitor targeting FAK, can improve survival of glioma xenografts in vivo, and disrupt glioma cell proliferation, attachment, migration and invasion in vitro [66]. In addition, this compound also induces breast cancer cell apoptosis in vitro [67]. Another small molecular FAK inhibitor, PF-652.271 is able to inhibit tumor growth and angiogenesis[68]. Thus, targeting FAK provides a prospective approach for cancer therapy.

Since playing an important role in tumorigenesis several FAK inhibitors have been developed. However, the recent inhibitors target the kinase activities of FAK that may introduce side effect[68]. In our study we use a different approach to regulate FAK activity by targeting the FAT domain to disrupt FAK focal adhesion localization. FAT is the protein interaction domain that contributes to FAK focal adhesion targeting through association with paxillin LD motifs. Structure shows the FAT domain containing two large hydrophobic patches for binding of LD motifs that are composed by helices H1/H4 and $\mathrm{H} 2 / \mathrm{H} 3$. Designing a inhibitor to target these hydrophobic patches become extremely difficult limited by the size of small molecules. A recently developed protein engineering technique provides the opportunity to explore a modified peptide to be used as peptidomimetics inhibitor for biological functions. This technique uses both covalent and noncovalent bonds to constrain peptides, forcing them into designed protein secondary structures. In this case the LD4 motif has been selected to perform the helical constraining for enhancing the binding affinity to FAT.

Previously our lab showed the LD4 motif forms the alpha helix structure upon binding while it remains unstructured in solution. $\alpha$-Helices have been found to be the secondary structure about $40 \%$ of all residues in natural proteins structure formations, and they are widely used as fundamental recognition elements in many naturally occurring protein complexes[235]. A typical alpha helix ends one rotation containing 3.6 amino acid residues with backbone dihedral angles of $\Psi=-41^{\circ}$ and $\Phi=-62^{\circ}$. This results in the helix having a rise of $1.5 \AA$ /residue or $5.4 \AA$ turn. Therefore, the side chain of a certain residue at the position projects from the same face with the side chains at the $i+4$ and the $i+7$ positions in the sequence. The backbone of the alpha helix is mainly stabilized by hydrogen bonds between the carbonyl of residues $i$ and the carboamide of 
residues $i+4$ that all point in the same direction. Because the hydrogen bonding sites on the first and last turns of an $\alpha$-helix are unfulfilled, this results in a dynamic helical structure. The helical constrained technique can efficiently stablize and maintain the alpha helix formation by cross-linking the side chains of the residue $i$ and residue $i+4$. In this case we observed best binding results from the LD4-L6A peptide, which cross-linked the residue Leu 6 and its $i+4$ residue Ala 10 by hydrocarbon stapling.

Although helical constrained techniques have been wildly accepted and used since it was developed, the design of cross-linked peptides follows the same rule, which is placing the linker away from the binding surface to avoid any potential clash. However, few studies report that hydrocarbon linkers can improve binding by increasing hydrophobicity[236]. Therefore, we are the first to design the LD4-L6A peptide to have the linker inserting into the hydrophobic binding patch with direct contact of target protein. The success of the LD4-L6A peptide provides new possibilities for designing constrained peptides in future.

\subsection{In Vivo Study of LD4-L6A}

Although we have prooved LD4-L6A can inhibit FAK/paxillin complex by targeting the FAT domain in vitro using fluorescent measurement, ITC and NMR CSP, the in vivo test needs to be preformed. Unlike in vitro studies the only factor is binding affinity to target proteins, that the permeability, stability, resistance to metabolism, and less side effects become key features of mature drug-like inhibitors. We have initiated a cell based assay as in vivo study of LD4-L6A targeting FAK/paxillin complex. We successfully observed helical constrained peptide could penetrate the cell membrane and co-localize with FAs, shown in Figure 5-1. However, further experiments need to be carried out to evaluate other factors of LD4-L6A as an inhibitor in vivo.

\subsection{The Complex Structure of P130Cas CCHD with Paxillin LD1 Motif}

The second half presented in this dissertation is elucidating the structure of the CCHD of p130Cas complex with LD1 motif of paxillin. The significance of the CCHD for the p130Cas FAs localization as well as the importance of the p130Cas function in FAs and its role in diseases has been discussed in previous chapters. It is well established that SH3 domains of p130Cas interacts with FAK through its N-terminal FERM domain. Similar to FAK, the p130Cas localizes to FAs and is phosphorylated by tyrosine kinase in response to adhesion signals [141-143]. A primary role of p130Cas in FA signal transduction is a signaling component that brings cellular responses to integrin engagement $[144,145]$. p130Cas has been demonstrated to be responsible for tamoxifen resistance in estrogen receptor positive breast cancer [146]. Therefore, the human homolog of p130Cas is known as well. The mechanism of anti-estrogen resistance studies provide solid evidence that p130Cas plays an important role in promoting cell proliferation and survival [147-150]. 


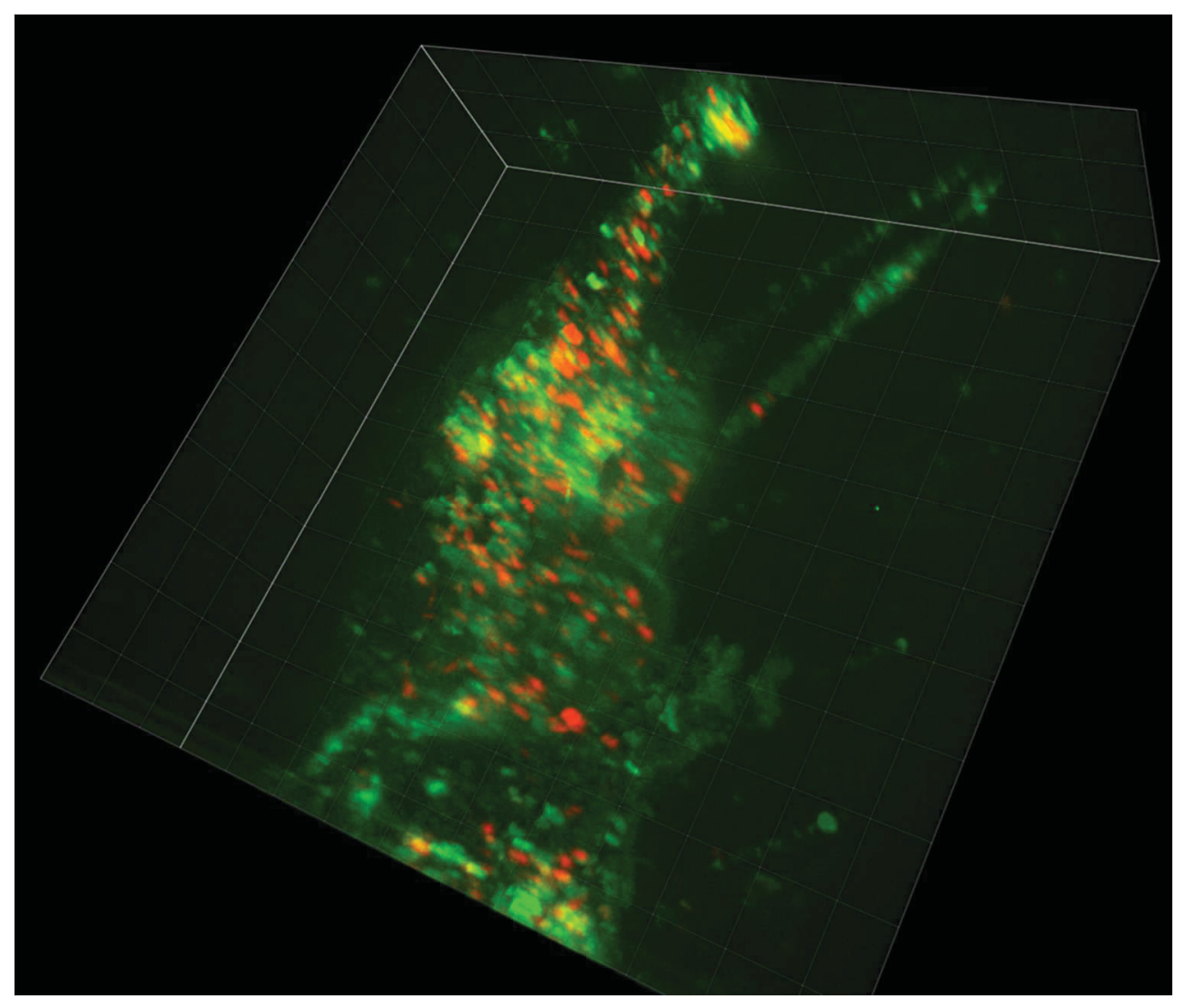

Figure 5-1. Confocal microscopy of helical constrained LD4-S12K addition to NIH$3 \mathrm{t} 3$ cell.

The cell membrane was fluorescently labeled by green color, and ROX-LD4-S12K colored in red was added to cell culture medium. Yellow color indicates that the ROXLD4-S12K penetrated the cell membrane and colocalized with FAs. 
Recent studies showed CCHD located at C-terminal of p130Cas plays an equally important role as the SH3 domain in its FAs targeting[145]. Lack of structural information raised difficulties associated with understanding the detailed mechanism of CCHD localizing p130Cas in FAs. CCHD has been predicted to adopt a similar structural fold as the FAT domain of FAK and it is proposed as a FAT-like domain in function. The structure of CCHD has been solved and revealed in complex with the NSP3 protein[230]. However, how CCHD facilitates p130Cas in FAs targeting remained unknown. Although adopting a similar structure as FAT that consists of four alpha helices forming the helix bundle, CCHD is unlike other FAT or FAT-like domain, such as FAK-FAT, PYK2-FAT and GIT1-FAH domain, that forms stable folded helix bundle in solution. It is impossible to isolate and purify the CCHD for searching for a binding partner from FAs. I identified an extended potential helical region containing a Src binding motif localized at the Nterminal end of CCHD. Introducing this region into the CCHD construct has dramatically increased the stability and solubility of CCHD. By this approach I successfully expressed and purified the elongated construct p130Cas ${ }^{665-874}$ containing both the the Scr binding motif and CCHD.

Through carefully analyzing the structure of CCHD, the data suggests that it is composed two hydrophobic patches comparing similarly with other FAT and FAT-like domains. Therefore I selected paxillin, which is the FAs marker that associates with FAT and FAT-like domains, as the candidate to identify the binding partner of CCHD in FAs. I have successfully observed association of CCHD with the LD1 motif of paxillin and this is a novel interaction that reveals how CCHD localizes p130Cas on FAs at the molecular level. Superimposing our CCHD/LD1 complex structure with the NSP3/Cas complex structure showed both structures of helix bundles are identical and the binding portion of NSP3 to CCHD overlaid well with LD1 to the $\mathrm{H} 2 / \mathrm{H} 3$ site of CCHD sharing the same binding mode shown in Figure 5-2. This further confirms our CCHD/LD1 complex structure.

\subsection{Unanswered Question of Regulating P130Cas Activity Through CCHD}

It has been well known that p130Cas targets FAs through its $\mathrm{SH} 3$ domain associating with FAK [141-143]. Recently, studies showed the CCHD of p130Cas plays an equally important role in p130Cas FAs localization, which has been confirmed by our structural study. From our structure we demonstrated helices $\mathrm{H} 2 / \mathrm{H} 3$ of CCHD composed a hydrophobic pocket for paxillin LD1 binding that facilitates p130Cas FAs targeting.

The complex structure of CCHD/LD1 indicates CCHD only contains one binding site compare with the FAT of FAK, which the H1/H4 hydrophobic patch with a switched charge residue on sidewall is not favorable for binding of LD motifs. This leaves a question whether the $\mathrm{H} 1 / \mathrm{H} 4$ site can interact with other binding partners in addition to the $\mathrm{LD} 1$ to $\mathrm{H} 2 / \mathrm{H} 3$ site that may play a regulatory role for p130Cas FAs activity. Because of the difficulty of expressing and and purifying CCHD domain we had to clone a new construct in order to verify our hypothesis. We may repeat our strategy of subcloning the FAT-LD2 construct to covalently link the LD1 motif onto CCHD stabilizing the helix 

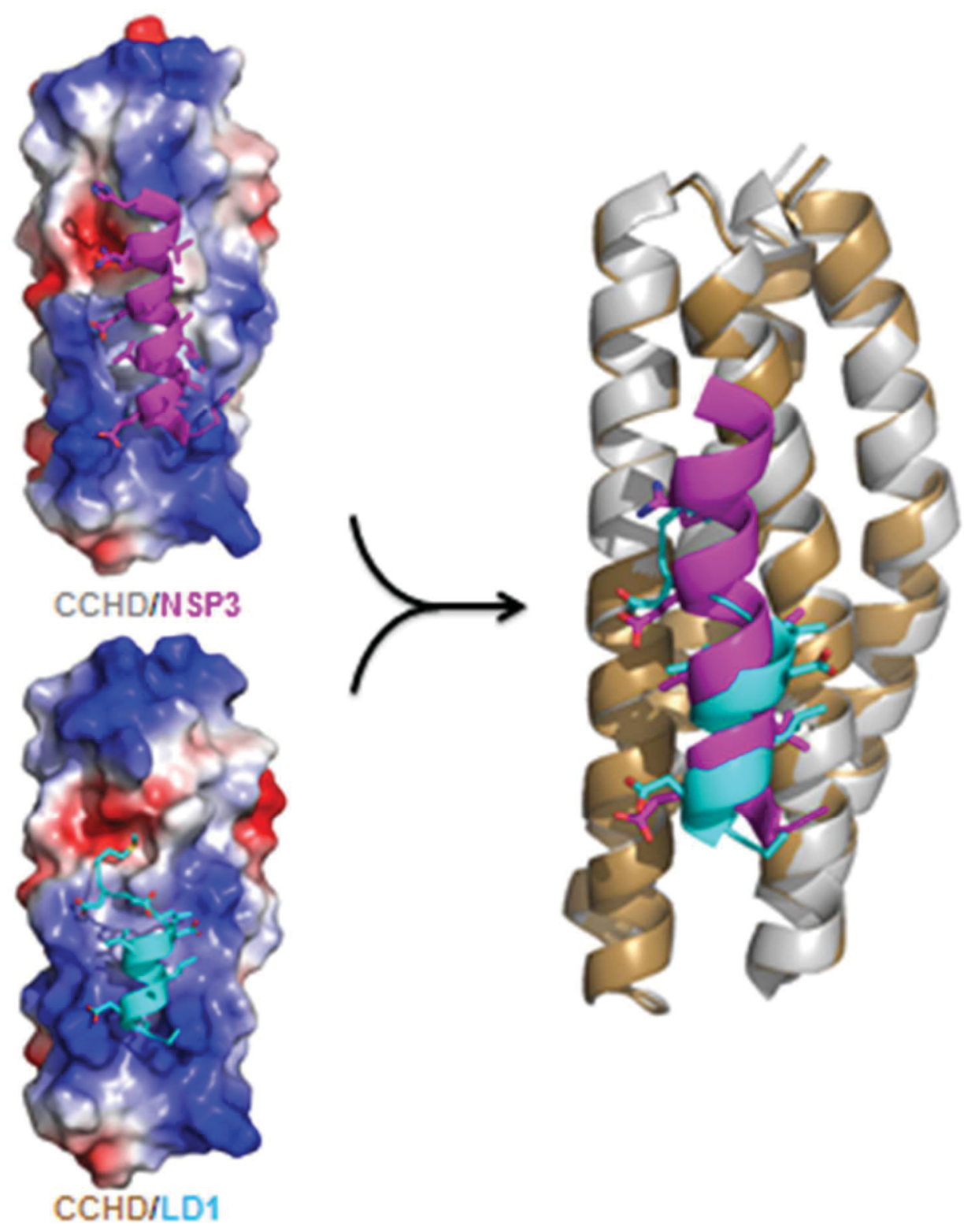

Figure 5-2. Comparing the CCHD/LD1 structure with CCHD/NSP3.

The CCHD domain and NSP3 binding portion in CCHD/NSP3 structure are colored by grey and magenta, while the CCHD and LD1 in CCHD/LD1 structure are colored by gold and cyan, respectively. Superimposing the two structure show the identical backbone arrangement and the LD1 peptide binds to $\mathrm{H} 2 / \mathrm{H} 3$ sharing the same binding mode as NSP3 peptide. 
bundle for identifying additional binding partners.

Second, we intentionally included the $\mathrm{N}$-terminal extension region containing a Src binding motif to stabilize the CCHD and indeed we successfully expressed and purified CCHD by introducing the elongated sequence into the construct. Maybe this region plays a regulatory role of CCHD by binding the Src binding site with CCHD by self-inhibition. However, the spectra show this region is unstructured and the structure of Src binding site interacting with CCHD cannot be obtained. I subcloned CCHD covalently linked this Src binding site at its N-terminal for obtaining the structural information, but all trials have failed. The length of the linker may be the key factor to express a stable fusion protein in the future.

Previous studies showed the p130Cas targets the FAs through the binding of its SH3 domain to FAK and FAK localized in FAs through the interaction with FAT to LD2 and LD4 motifs of paxillin. Our structure revealed the CCHD of p130Cas plays an equally important role on p130Cas FAs targeting by binding of CCHD with LD1. Together I hypothesize that FAK/p130Cas/paxillin can form a super-complex with the SH3 associating with FAK, and both FAT and CCHD interact with paxillin LD2/LD4 and LD1 respectively. Further data is need for verifying this model. 


\section{LIST OF REFERENCES}

1. Friedl, P. and K. Wolf, Plasticity of cell migration: a multiscale tuning model. J Cell Biol, 2010. 188(1): p. 11-9.

2. Ridley, A.J., et al., Cell migration: integrating signals from front to back. Science, 2003. 302(5651): p. 1704-9.

3. Webb, D.J., J.T. Parsons, and A.F. Horwitz, Adhesion assembly, disassembly and turnover in migrating cells -- over and over and over again. Nat Cell Biol, 2002. 4(4): p. E97-100.

4. Funamoto, S., et al., Spatial and temporal regulation of 3-phosphoinositides by PI 3-kinase and PTEN mediates chemotaxis. Cell, 2002. 109(5): p. 611-23.

5. Srinivasan, S., et al., Rac and Cdc42 play distinct roles in regulating $P I(3,4,5) P 3$ and polarity during neutrophil chemotaxis. J Cell Biol, 2003. 160(3): p. 375-85.

6. $\quad$ Rodriguez, O.C., et al., Conserved microtubule-actin interactions in cell movement and morphogenesis. Nat Cell Biol, 2003. 5(7): p. 599-609.

7. Welch, H.C., et al., Phosphoinositide 3-kinase-dependent activation of Rac. FEBS Lett, 2003. 546(1): p. 93-7.

8. Etienne-Manneville, S. and A. Hall, Integrin-mediated activation of Cdc42 controls cell polarity in migrating astrocytes through PKCzeta. Cell, 2001. 106(4): p. 489-98.

9. Wilson, K., et al., Mechanisms of leading edge protrusion in interstitial migration. Nat Commun, 2013. 4: p. 2896.

10. Weaver, A.M., et al., Integration of signals to the Arp2/3 complex. Curr Opin Cell Biol, 2003. 15(1): p. 23-30.

11. Vartiainen, M.K. and L.M. Machesky, The WASP-Arp2/3 pathway: genetic insights. Curr Opin Cell Biol, 2004. 16(2): p. 174-81.

12. Watanabe, N. and C. Higashida, Formins: processive cappers of growing actin filaments. Exp Cell Res, 2004. 301(1): p. 16-22.

13. Hynes, R.O., Integrins: bidirectional, allosteric signaling machines. Cell, 2002. 110(6): p. 673-87.

14. Campbell, I.D. and M.J. Humphries, Integrin structure, activation, and interactions. Cold Spring Harb Perspect Biol, 2011. 3(3).

15. Geiger, B., et al., Transmembrane crosstalk between the extracellular matrix-cytoskeleton crosstalk. Nat Rev Mol Cell Biol, 2001. 2(11): p. 793-805.

16. Verkhovsky, A.B., T.M. Svitkina, and G.G. Borisy, Network contraction model for cell translocation and retrograde flow. Biochem Soc Symp, 1999. 65: p. 20722.

17. Cramer, L.P., Molecular mechanism of actin-dependent retrograde flow in lamellipodia of motile cells. Front Biosci, 1997. 2: p. d260-70.

18. Jay, P.Y., et al., A mechanical function of myosin II in cell motility. J Cell Sci, 1995. 108 ( Pt 1): p. 387-93.

19. Verkhovsky, A.B., T.M. Svitkina, and G.G. Borisy, Self-polarization and directional motility of cytoplasm. Curr Biol, 1999. 9(1): p. 11-20.

20. Worthylake, R.A. and K. Burridge, RhoA and ROCK promote migration by limiting membrane protrusions. J Biol Chem, 2003. 278(15): p. 13578-84. 
21. $\mathrm{Xu}, \mathrm{J}$., et al., Divergent signals and cytoskeletal assemblies regulate selforganizing polarity in neutrophils. Cell, 2003. 114(2): p. 201-14.

22. Chung, C.Y., G. Potikyan, and R.A. Firtel, Control of cell polarity and chemotaxis by Akt/PKB and PI3 kinase through the regulation of PAKa. Mol Cell, 2001. 7(5): p. 937-47.

23. Vicente-Manzanares, M., et al., Regulation of protrusion, adhesion dynamics, and polarity by myosins IIA and IIB in migrating cells. J Cell Biol, 2007. 176(5): p. 573-80.

24. Vicente-Manzanares, M., et al., Myosin IIA/IIB restrict adhesive and protrusive signaling to generate front-back polarity in migrating cells. J Cell Biol, 2011. 193(2): p. 381-96.

25. Laukaitis, C.M., et al., Differential dynamics of alpha 5 integrin, paxillin, and alpha-actinin during formation and disassembly of adhesions in migrating cells. $\mathrm{J}$ Cell Biol, 2001. 153(7): p. 1427-40.

26. Kirfel, G., et al., Cell migration: mechanisms of rear detachment and the formation of migration tracks. Eur J Cell Biol, 2004. 83(11-12): p. 717-24.

27. Zaidel-Bar, R., et al., Hierarchical assembly of cell-matrix adhesion complexes. Biochem Soc Trans, 2004. 32(Pt3): p. 416-20.

28. Lo, S.H., Focal adhesions: what's new inside. Dev Biol, 2006. 294(2): p. 280-91.

29. Jockusch, B.M., et al., The molecular architecture of focal adhesions. Annu Rev Cell Dev Biol, 1995. 11: p. 379-416.

30. Liu, G., C.D. Guibao, and J. Zheng, Structural insight into the mechanisms of targeting and signaling of focal adhesion kinase. Mol Cell Biol, 2002. 22(8): p. 2751-60.

31. Giancotti, F.G. and E. Ruoslahti, Integrin signaling. Science, 1999. 285(5430): p. 1028-32.

32. Beglova, N., et al., Cysteine-rich module structure reveals a fulcrum for integrin rearrangement upon activation. Nat Struct Biol, 2002. 9(4): p. 282-7.

33. Shimaoka, M., et al., Stabilizing the integrin alpha M inserted domain in alternative conformations with a range of engineered disulfide bonds. Proc Natl Acad Sci U S A, 2002. 99(26): p. 16737-41.

34. Zhao, Z.S., et al., Coupling of PAK-interacting exchange factor PIX to GIT1 promotes focal complex disassembly. Mol Cell Biol, 2000. 20(17): p. 6354-63.

35. Manser, E., et al., Expression of constitutively active alpha-PAK reveals effects of the kinase on actin and focal complexes. Mol Cell Biol, 1997. 17(3): p. 1129-43.

36. Moro, L., et al., Integrin-induced epidermal growth factor (EGF) receptor activation requires $\mathrm{c}-\mathrm{Src}$ and $130 \mathrm{Cas}$ and leads to phosphorylation of specific EGF receptor tyrosines. J Biol Chem, 2002. 277(11): p. 9405-14.

37. Borges, E., Y. Jan, and E. Ruoslahti, Platelet-derived growth factor receptor beta and vascular endothelial growth factor receptor 2 bind to the beta 3 integrin through its extracellular domain. J Biol Chem, 2000. 275(51): p. 39867-73.

38. Schneller, M., K. Vuori, and E. Ruoslahti, Alphavbeta3 integrin associates with activated insulin and PDGFbeta receptors and potentiates the biological activity of PDGF. EMBO J, 1997. 16(18): p. 5600-7.

39. Hood, J.D., et al., Differential alphav integrin-mediated Ras-ERK signaling during two pathways of angiogenesis. J Cell Biol, 2003. 162(5): p. 933-43. 
40. Breitschopf, K., et al., Posttranslational modification of Bcl-2 facilitates its proteasome-dependent degradation: molecular characterization of the involved signaling pathway. Mol Cell Biol, 2000. 20(5): p. 1886-96.

41. Stromblad, S., et al., Suppression of p53 activity and p21WAF1/CIP1 expression by vascular cell integrin alphaVbeta3 during angiogenesis. J Clin Invest, 1996. 98(2): p. 426-33.

42. Vivanco, I. and C.L. Sawyers, The phosphatidylinositol 3-Kinase AKT pathway in human cancer. Nat Rev Cancer, 2002. 2(7): p. 489-501.

43. Nave, B.T., et al., Mammalian target of rapamycin is a direct target for protein kinase B: identification of a convergence point for opposing effects of insulin and amino-acid deficiency on protein translation. Biochem J, 1999. 344 Pt 2: p. 42731.

44. Schaller, M.D., et al., pp 125FAK a structurally distinctive protein-tyrosine kinase associated with focal adhesions. Proc Natl Acad Sci U S A, 1992. 89(11): p. 5192-6.

45. Tilghman, R.W. and J.T. Parsons, Focal adhesion kinase as a regulator of cell tension in the progression of cancer. Semin Cancer Biol, 2008. 18(1): p. 45-52.

46. Mitra, S.K., D.A. Hanson, and D.D. Schlaepfer, Focal adhesion kinase: in command and control of cell motility. Nat Rev Mol Cell Biol, 2005. 6(1): p. 5668.

47. Cooper, L.A., T.L. Shen, and J.L. Guan, Regulation of focal adhesion kinase by its amino-terminal domain through an autoinhibitory interaction. Mol Cell Biol, 2003. 23(22): p. 8030-41.

48. Sieg, D.J., et al., FAK integrates growth-factor and integrin signals to promote cell migration. Nat Cell Biol, 2000. 2(5): p. 249-56.

49. van Nimwegen, M.J. and B. van de Water, Focal adhesion kinase: a potential target in cancer therapy. Biochem Pharmacol, 2007. 73(5): p. 597-609.

50. Chan, P.Y., et al., A transmembrane-anchored chimeric focal adhesion kinase is constitutively activated and phosphorylated at tyrosine residues identical to pp125FAK. J Biol Chem, 1994. 269(32): p. 20567-74.

51. Schlaepfer, D.D. and T. Hunter, Evidence for in vivo phosphorylation of the Grb2 SH2-domain binding site on focal adhesion kinase by Src-family protein-tyrosine kinases. Mol Cell Biol, 1996. 16(10): p. 5623-33.

52. Lietha, D., et al., Structural basis for the autoinhibition of focal adhesion kinase. Cell, 2007. 129(6): p. 1177-87.

53. Toutant, M., et al., Alternative splicing controls the mechanisms of FAK autophosphorylation. Mol Cell Biol, 2002. 22(22): p. 7731-43.

54. Hanks, S.K., et al., Focal adhesion kinase signaling activities and their implications in the control of cell survival and motility. Front Biosci, 2003. 8: p. d982-96.

55. Chodniewicz, D. and R.L. Klemke, Regulation of integrin-mediated cellular responses through assembly of a CAS/Crk scaffold. Biochim Biophys Acta, 2004. 1692(2-3): p. 63-76.

56. Turner, C.E., Paxillin and focal adhesion signalling. Nat Cell Biol, 2000. 2(12): p. E231-6. 
57. Schaller, M.D., Biochemical signals and biological responses elicited by the focal adhesion kinase. Biochim Biophys Acta, 2001. 1540(1): p. 1-21.

58. Parsons, J.T., Focal adhesion kinase: the first ten years. J Cell Sci, 2003. 116(Pt 8): p. 1409-16.

59. Hildebrand, J.D., M.D. Schaller, and J.T. Parsons, Identification of sequences required for the efficient localization of the focal adhesion kinase, pp $125 \mathrm{FAK}$, to cellular focal adhesions. J Cell Biol, 1993. 123(4): p. 993-1005.

60. Martin, K.H., S.A. Boerner, and J.T. Parsons, Regulation of focal adhesion targeting and inhibitory functions of the FAK related protein FRNK using a novel estrogen receptor "switch". Cell Motil Cytoskeleton, 2002. 51(2): p. 76-88.

61. Golubovskaya, V.M., F.A. Kweh, and W.G. Cance, Focal adhesion kinase and cancer. Histol Histopathol, 2009. 24(4): p. 503-10.

62. $\mathrm{Xu}, \mathrm{L} . \mathrm{H}$. , et al., Attenuation of the expression of the focal adhesion kinase induces apoptosis in tumor cells. Cell Growth Differ, 1996. 7(4): p. 413-8.

63. Lipinski, C.A., et al., Differential role of proline-rich tyrosine kinase 2 and focal adhesion kinase in determining glioblastoma migration and proliferation. Mol Cancer Res, 2003. 1(5): p. 323-32.

64. Lipinski, C.A., et al., The tyrosine kinase pyk2 promotes migration and invasion of glioma cells. Neoplasia, 2005. 7(5): p. 435-45.

65. Wu, Z.M., et al., Antisense oligonucleodes targeting the focal adhesion kinase inhibit proliferation, induce apoptosis and cooperate with cytotoxic drugs in human glioma cells. J Neurooncol, 2006. 77(2): p. 117-23.

66. Liu, T.J., et al., Inhibition of both focal adhesion kinase and insulin-like growth factor-I receptor kinase suppresses glioma proliferation in vitro and in vivo. Mol Cancer Ther, 2007. 6(4): p. 1357-67.

67. Golubovskaya, V.M., C. Virnig, and W.G. Cance, TAE226-induced apoptosis in breast cancer cells with overexpressed Src or EGFR. Mol Carcinog, 2008. 47(3): p. 222-34.

68. Roberts, W.G., et al., Antitumor activity and pharmacology of a selective focal adhesion kinase inhibitor, PF-562,271. Cancer Res, 2008. 68(6): p. 1935-44.

69. Turner, C.E., J.R. Glenney, Jr., and K. Burridge, Paxillin: a new vinculin-binding protein present in focal adhesions. J Cell Biol, 1990. 111(3): p. 1059-68.

70. Perez-Alvarado, G.C., et al., Structure of the carboxy-terminal LIM domain from the cysteine rich protein CRP. Nat Struct Biol, 1994. 1(6): p. 388-98.

71. Schmeichel, K.L. and M.C. Beckerle, The LIM domain is a modular proteinbinding interface. Cell, 1994. 79(2): p. 211-9.

72. Brown, M.C., J.A. Perrotta, and C.E. Turner, Identification of LIM3 as the principal determinant of paxillin focal adhesion localization and characterization of a novel motif on paxillin directing vinculin and focal adhesion kinase binding. $\mathrm{J}$ Cell Biol, 1996. 135(4): p. 1109-23.

73. Liu, S., et al., Binding of paxillin to alpha4 integrins modifies integrin-dependent biological responses. Nature, 1999. 402(6762): p. 676-81.

74. Brown, M.C., J.A. Perrotta, and C.E. Turner, Serine and threonine phosphorylation of the paxillin LIM domains regulates paxillin focal adhesion localization and cell adhesion to fibronectin. Mol Biol Cell, 1998. 9(7): p. 180316. 
75. Cote, J.F., C.E. Turner, and M.L. Tremblay, Intact LIM 3 and LIM 4 domains of paxillin are required for the association to a novel polyproline region (Pro 2) of protein-tyrosine phosphatase-PEST. J Biol Chem, 1999. 274(29): p. 20550-60.

76. Herreros, L., et al., Paxillin localizes to the lymphocyte microtubule organizing center and associates with the microtubule cytoskeleton. J Biol Chem, 2000. 275(34): p. 26436-40.

77. Webb, D.J., et al., FAK-Src signalling through paxillin, ERK and MLCK regulates adhesion disassembly. Nat Cell Biol, 2004. 6(2): p. 154-61.

78. Efimov, A., et al., Paxillin-dependent stimulation of microtubule catastrophes at focal adhesion sites. J Cell Sci, 2008. 121(Pt 2): p. 196-204.

79. Brown, M.C. and C.E. Turner, Paxillin: adapting to change. Physiol Rev, 2004. 84(4): p. 1315-39.

80. Turner, C.E. and J.T. Miller, Primary sequence of paxillin contains putative SH2 and SH3 domain binding motifs and multiple LIM domains: identification of a vinculin and pp125Fak-binding region. J Cell Sci, 1994. 107 ( Pt 6): p. 1583-91.

81. Tumbarello, D.A., M.C. Brown, and C.E. Turner, The paxillin LD motifs. FEBS Lett, 2002. 513(1): p. 114-8.

82. Brown, M.C., M.S. Curtis, and C.E. Turner, Paxillin LD motifs may define a new family of protein recognition domains. Nat Struct Biol, 1998. 5(8): p. 677-8.

83. Shola, D.T., et al., Hic-5 controls BMP4 responses in prostate cancer cells through interacting with Smads 1, 5 and 8. Oncogene, 2012. 31(19): p. 2480-90.

84. Sheibani, N., Y. Tang, and C.M. Sorenson, Paxillin's LD4 motif interacts with bcl-2. J Cell Physiol, 2008. 214(3): p. 655-61.

85. Woods, A.J., et al., Paxillin associates with poly(A)-binding protein 1 at the dense endoplasmic reticulum and the leading edge of migrating cells. J Biol Chem, 2002. 277(8): p. 6428-37.

86. Hoellerer, M.K., et al., Molecular recognition of paxillin LD motifs by the focal adhesion targeting domain. Structure, 2003. 11(10): p. 1207-17.

87. Deakin, N.O. and C.E. Turner, Paxillin comes of age. J Cell Sci, 2008. 121(Pt 15): p. 2435-44.

88. Butler, A.A., et al., In vivo regulation of CrkII and CrkL proto-oncogenes in the uterus by insulin-like growth factor-I. Differential effects on tyrosine phosphorylation and association with paxillin. J Biol Chem, 1997. 272(44): p. 27660-4.

89. Ryu, H., et al., Regulation of neutrophil adhesion by pituitary growth hormone accompanies tyrosine phosphorylation of Jak2, p125FAK, and paxillin. $\mathrm{J}$ Immunol, 2000. 165(4): p. 2116-23.

90. Tapia, J.A., et al., EGF stimulates tyrosine phosphorylation of focal adhesion kinase (p125FAK) and paxillin in rat pancreatic acini by a phospholipase $C$ independent process that depends on phosphatidylinositol 3-kinase, the small GTP-binding protein, p21rho, and the integrity of the actin cytoskeleton. Biochim Biophys Acta, 1999. 1448(3): p. 486-99.

91. Rankin, S., et al., Requirement for phosphatidylinositol 3'-kinase activity in platelet-derived growth factor-stimulated tyrosine phosphorylation of p125 focal adhesion kinase and paxillin. J Biol Chem, 1996. 271(13): p. 7829-34. 
92. Abedi, H. and I. Zachary, Vascular endothelial growth factor stimulates tyrosine phosphorylation and recruitment to new focal adhesions of focal adhesion kinase and paxillin in endothelial cells. J Biol Chem, 1997. 272(24): p. 15442-51.

93. Zhong, H. and K.P. Minneman, Activation of tyrosine kinases by alphalAadrenergic and growth factor receptors in transfected PC12 cells. Biochem J, 1999. 344 Pt 3: p. 889-94.

94. Turner, C.E., et al., Angiotensin II stimulation of rapid paxillin tyrosine phosphorylation correlates with the formation of focal adhesions in rat aortic smooth muscle cells. J Cell Sci, 1995. 108 ( Pt 1): p. 333-42.

95. Doucey, M.A., et al., The betal and beta3 integrins promote T cell receptormediated cytotoxic T lymphocyte activation. J Biol Chem, 2003. 278(29): p. 26983-91.

96. Hamawy, M.M., et al., The aggregation of the high affinity IgE receptor induces tyrosine phosphorylation of paxillin, a focal adhesion protein. J Immunol, 1994. 153(10): p. 4655-62.

97. Tsubouchi, A., et al., Localized suppression of RhoA activity by Tyr31/118phosphorylated paxillin in cell adhesion and migration. J Cell Biol, 2002. 159(4): p. 673-83.

98. Grgurevich, S., A. Mikhael, and D.W. McVicar, The Csk homologous kinase, Chk, binds tyrosine phosphorylated paxillin in human blastic T cells. Biochem Biophys Res Commun, 1999. 256(3): p. 668-75.

99. De Nichilo, M.O. and K.M. Yamada, Integrin alpha $v$ beta 5-dependent serine phosphorylation of paxillin in cultured human macrophages adherent to vitronectin. J Biol Chem, 1996. 271(18): p. 11016-22.

100. Bellis, S.L., et al., Adhesion of fibroblasts to fibronectin stimulates both serine and tyrosine phosphorylation of paxillin. Biochem J, 1997. 325 ( Pt 2): p. 375-81.

101. Riedy, M.C., et al., Activin A and TGF-beta stimulate phosphorylation of focal adhesion proteins and cytoskeletal reorganization in rat aortic smooth muscle cells. Exp Cell Res, 1999. 251(1): p. 194-202.

102. Brown, M.C. and C.E. Turner, Characterization of paxillin LIM domainassociated serine threonine kinases: activation by angiotensin II in vascular smooth muscle cells. J Cell Biochem, 1999. 76(1): p. 99-108.

103. Yamaguchi, R., et al., Mitosis specific serine phosphorylation and downregulation of one of the focal adhesion protein, paxillin. Oncogene, 1997. 15(15): p. 1753-61.

104. Terfera, D.R., M.C. Brown, and C.E. Turner, Epidermal growth factor stimulates serine/threonine phosphorylation of the focal adhesion protein paxillin in a MEKdependent manner in normal rat kidney cells. J Cell Physiol, 2002. 191(1): p. 8294.

105. Ishibe, S., et al., Phosphorylation-dependent paxillin-ERK association mediates hepatocyte growth factor-stimulated epithelial morphogenesis. Mol Cell, 2003. 12(5): p. 1275-85.

106. Liu, Z.X., et al., Hepatocyte growth factor induces ERK-dependent paxillin phosphorylation and regulates paxillin-focal adhesion kinase association. J Biol Chem, 2002. 277(12): p. 10452-8. 
107. Hashimoto, S., et al., Interaction of paxillin with p21-activated Kinase (PAK). Association of paxillin alpha with the kinase-inactive and the Cdc42-activated forms of PAK3. J Biol Chem, 2001. 276(8): p. 6037-45.

108. Wera, S. and B.A. Hemmings, Serine/threonine protein phosphatases. Biochem J, 1995. 311 ( Pt 1): p. 17-29.

109. Brown, M.C. and C.E. Turner, Roles for the tubulin- and PTP-PEST-binding paxillin LIM domains in cell adhesion and motility. Int J Biochem Cell Biol, 2002. 34(7): p. 855-63.

110. Ito, A., et al., A truncated isoform of the PP2A B56 subunit promotes cell motility through paxillin phosphorylation. EMBO J, 2000. 19(4): p. 562-71.

111. Hanna, S. and M. El-Sibai, Signaling networks of Rho GTPases in cell motility. Cell Signal, 2013. 25(10): p. 1955-61.

112. Schaller, M.D. and J.T. Parsons, pp125FAK-dependent tyrosine phosphorylation of paxillin creates a high-affinity binding site for Crk. Mol Cell Biol, 1995. 15(5): p. 2635-45.

113. Lu, M. and K.S. Ravichandran, Dock180-ELMO cooperation in Rac activation. Methods Enzymol, 2006. 406: p. 388-402.

114. West, K.A., et al., The LD4 motif of paxillin regulates cell spreading and motility through an interaction with paxillin kinase linker (PKL). J Cell Biol, 2001. 154(1): p. 161-76.

115. Turner, C.E., et al., Paxillin LD4 motif binds PAK and PIX through a novel 95-kD ankyrin repeat, ARF-GAP protein: A role in cytoskeletal remodeling. J Cell Biol, 1999. 145(4): p. 851-63.

116. Premont, R.T., et al., The GIT family of ADP-ribosylation factor GTPaseactivating proteins. Functional diversity of GIT2 through alternative splicing. J Biol Chem, 2000. 275(29): p. 22373-80.

117. Premont, R.T., et al., The GIT/PIX complex: an oligomeric assembly of GIT family ARF GTPase-activating proteins and PIX family Rac1/Cdc42 guanine nucleotide exchange factors. Cell Signal, 2004. 16(9): p. 1001-11.

118. Petros, A.M., et al., Solution structure of the antiapoptotic protein bcl-2. Proc Natl Acad Sci U S A, 2001. 98(6): p. 3012-7.

119. Izard, T., et al., Vinculin activation by talin through helical bundle conversion. Nature, 2004. 427(6970): p. 171-5.

120. Schmalzigaug, R., et al., GIT1 utilizes a focal adhesion targeting-homology domain to bind paxillin. Cell Signal, 2007. 19(8): p. 1733-44.

121. Hayashi, I., K. Vuori, and R.C. Liddington, The focal adhesion targeting (FAT) region of focal adhesion kinase is a four-helix bundle that binds paxillin. Nat Struct Biol, 2002. 9(2): p. 101-6.

122. Subauste, M.C., et al., Vinculin modulation of paxillin-FAK interactions regulates ERK to control survival and motility. J Cell Biol, 2004. 165(3): p. 371-81.

123. Chay, K.O., S.S. Park, and J.F. Mushinski, Linkage of caspase-mediated degradation of paxillin to apoptosis in Ba/F3 murine pro-B lymphocytes. J Biol Chem, 2002. 277(17): p. 14521-9.

124. Hagel, M., et al., The adaptor protein paxillin is essential for normal development in the mouse and is a critical transducer of fibronectin signaling. Mol Cell Biol, 2002. 22(3): p. 901-15. 
125. Mackinnon, A.C., et al., Paxillin expression and amplification in early lung lesions of high-risk patients, lung adenocarcinoma and metastatic disease. J Clin Pathol, 2011. 64(1): p. 16-24.

126. Jagadeeswaran, R., et al., Paxillin is a target for somatic mutations in lung cancer: implications for cell growth and invasion. Cancer Res, 2008. 68(1): p. 132-42.

127. Yu, J.A., N.O. Deakin, and C.E. Turner, Paxillin-kinase-linker tyrosine phosphorylation regulates directional cell migration. Mol Biol Cell, 2009. 20(22): p. 4706-19.

128. Ren, Y., et al., Roles of Gab1 and SHP2 in paxillin tyrosine dephosphorylation and Src activation in response to epidermal growth factor. J Biol Chem, 2004. 279(9): p. 8497-505.

129. Tatsumi, Y., et al., Involvement of the paxillin pathway in JB6 Cl41 cell transformation. Cancer Res, 2006. 66(11): p. 5968-74.

130. Zhao, Y., et al., Identification and functional characterization of paxillin as a target of protein tyrosine phosphatase receptor T. Proc Natl Acad Sci U S A, 2010. 107(6): p. 2592-7.

131. Kanteti, R., et al., MET, HGF, EGFR, and PXN gene copy number in lung cancer using DNA extracts from FFPE archival samples and prognostic significance. $\mathrm{J}$ Environ Pathol Toxicol Oncol, 2009. 28(2): p. 89-98.

132. Short, S.M., et al., The expression of the cytoskeletal focal adhesion protein paxillin in breast cancer correlates with HER 2 overexpression and may help predict response to chemotherapy: a retrospective immunohistochemical study. Breast J, 2007. 13(2): p. 130-9.

133. Madan, R., et al., Focal adhesion proteins as markers of malignant transformation and prognostic indicators in breast carcinoma. Hum Pathol, 2006. 37(1): p. 9-15.

134. Wang, Z., et al., Adeno-associated virus serotype 8 efficiently delivers genes to muscle and heart. Nat Biotechnol, 2005. 23(3): p. 321-8.

135. Kim, G., et al., Adhesion molecule protein signature in ovarian cancer effusions is prognostic of patient outcome. Cancer, 2012. 118(6): p. 1543-53.

136. Defilippi, P., P. Di Stefano, and S. Cabodi, p130Cas: a versatile scaffold in signaling networks. Trends Cell Biol, 2006. 16(5): p. 257-63.

137. Mayer, B.J., M. Hamaguchi, and H. Hanafusa, A novel viral oncogene with structural similarity to phospholipase C. Nature, 1988. 332(6161): p. 272-5.

138. Reynolds, A.B., et al., Stable association of activated pp60src with two tyrosinephosphorylated cellular proteins. Mol Cell Biol, 1989. 9(9): p. 3951-8.

139. Sakai, R., et al., A novel signaling molecule, p130, forms stable complexes in vivo with $v$-Crk and $v$-Src in a tyrosine phosphorylation-dependent manner. EMBO J, 1994. 13(16): p. 3748-56.

140. Polte, T.R. and S.K. Hanks, Interaction between focal adhesion kinase and Crkassociated tyrosine kinase substrate p130Cas. Proc Natl Acad Sci U S A, 1995. 92(23): p. 10678-82.

141. Petch, L.A., et al., Adhesion-induced tyrosine phosphorylation of the p130 src substrate. J Cell Sci, 1995. 108 ( Pt 4): p. 1371-9. 
142. Vuori, K. and E. Ruoslahti, Tyrosine phosphorylation of p130Cas and cortactin accompanies integrin-mediated cell adhesion to extracellular matrix. J Biol Chem, 1995. 270(38): p. 22259-62.

143. Nojima, Y., et al., Focal adhesion kinase (pp125FAK) is tyrosine phosphorylated after engagement of alpha 4 beta 1 and alpha 5 beta 1 integrins on human Tlymphoblastic cells. Cell Immunol, 1995. 161(1): p. 8-13.

144. Honda, H., et al., Cardiovascular anomaly, impaired actin bundling and resistance to Src-induced transformation in mice lacking p130Cas. Nat Genet, 1998. 19(4): p. 361-5.

145. Donato, D.M., et al., Dynamics and mechanism of p130Cas localization to focal adhesions. J Biol Chem, 2010. 285(27): p. 20769-79.

146. Brinkman, A., et al., BCAR1, a human homologue of the adapter protein p130Cas, and antiestrogen resistance in breast cancer cells. J Natl Cancer Inst, 2000. 92(2): p. 112-20.

147. Cabodi, S., et al., p130Cas interacts with estrogen receptor alpha and modulates non-genomic estrogen signaling in breast cancer cells. J Cell Sci, 2004. 117(Pt 8): p. 1603-11.

148. Cabodi, S., et al., p130Cas as a new regulator of mammary epithelial cell proliferation, survival, and HER2-neu oncogene-dependent breast tumorigenesis. Cancer Res, 2006. 66(9): p. 4672-80.

149. Riggins, R.B., et al., Physical and functional interactions between Cas and c-Src induce tamoxifen resistance of breast cancer cells through pathways involving epidermal growth factor receptor and signal transducer and activator of transcription 5b. Cancer Res, 2006. 66(14): p. 7007-15.

150. Soni, S., et al., Expression of a phosphorylated p130(Cas) substrate domain attenuates the phosphatidylinositol 3-kinase/Akt survival pathway in tamoxifen resistant breast cancer cells. J Cell Biochem, 2009. 107(2): p. 364-75.

151. Guerrero, M.S., J.T. Parsons, and A.H. Bouton, Cas and NEDD9 Contribute to Tumor Progression through Dynamic Regulation of the Cytoskeleton. Genes Cancer, 2012. 3(5-6): p. 371-81.

152. Tikhmyanova, N., J.L. Little, and E.A. Golemis, CAS proteins in normal and pathological cell growth control. Cell Mol Life Sci, 2010. 67(7): p. 1025-48.

153. Cary, L.A., et al., Identification of p130Cas as a mediator of focal adhesion kinase-promoted cell migration. J Cell Biol, 1998. 140(1): p. 211-21.

154. Astier, A., et al., The related adhesion focal tyrosine kinase differentially phosphorylates p130Cas and the Cas-like protein, p105HEF1. J Biol Chem, 1997. 272(32): p. 19719-24.

155. Kirsch, K.H., M.M. Georgescu, and H. Hanafusa, Direct binding of p130(Cas) to the guanine nucleotide exchange factor C3G. J Biol Chem, 1998. 273(40): p. 25673-9.

156. Kirsch, K.H., et al., CMS: an adapter molecule involved in cytoskeletal rearrangements. Proc Natl Acad Sci U S A, 1999. 96(11): p. 6211-6.

157. Chan, Y.R. and R.L. Gallo, PR-39, a syndecan-inducing antimicrobial peptide, binds and affects p130(Cas). J Biol Chem, 1998. 273(44): p. 28978-85. 
158. Garton, A.J., et al., Association of PTP-PEST with the SH3 domain of p130cas; a novel mechanism of protein tyrosine phosphatase substrate recognition. Oncogene, 1997. 15(8): p. 877-85.

159. Liu, F., M.A. Sells, and J. Chernoff, Protein tyrosine phosphatase $1 B$ negatively regulates integrin signaling. Curr Biol, 1998. 8(3): p. 173-6.

160. Nakamoto, T., et al., CIZ, a zinc finger protein that interacts with p130(cas) and activates the expression of matrix metalloproteinases. Mol Cell Biol, 2000. 20(5): p. 1649-58.

161. Harte, M.T., et al., p130Cas, a substrate associated with v-Src and v-Crk, localizes to focal adhesions and binds to focal adhesion kinase. J Biol Chem, 1996. 271(23): p. 13649-55.

162. Manie, S.N., et al., Involvement of p130(Cas) and p105(HEF1), a novel Cas-like docking protein, in a cytoskeleton-dependent signaling pathway initiated by ligation of integrin or antigen receptor on human B cells. J Biol Chem, 1997. 272(7): p. 4230-6.

163. Feller, S.M., Crk family adaptors-signalling complex formation and biological roles. Oncogene, 2001. 20(44): p. 6348-71.

164. Shih, N.Y., et al., Congenital nephrotic syndrome in mice lacking CD2-associated protein. Science, 1999. 286(5438): p. 312-5.

165. Liu, F., D.E. Hill, and J. Chernoff, Direct binding of the proline-rich region of protein tyrosine phosphatase $1 B$ to the Src homology 3 domain of p130(Cas). J Biol Chem, 1996. 271(49): p. 31290-5.

166. Cheng, A., et al., Attenuation of adhesion-dependent signaling and cell spreading in transformed fibroblasts lacking protein tyrosine phosphatase-1B. J Biol Chem, 2001. 276(28): p. 25848-55.

167. Liang, F., et al., The role of protein-tyrosine phosphatase $1 B$ in integrin signaling. J Biol Chem, 2005. 280(26): p. 24857-63.

168. Garton, A.J., A.J. Flint, and N.K. Tonks, Identification of p130(cas) as a substrate for the cytosolic protein tyrosine phosphatase PTP-PEST. Mol Cell Biol, 1996. 16(11): p. 6408-18.

169. Sakai, R., et al., Characterization of the kinase activity essential for tyrosine phosphorylation of p130Cas in fibroblasts. Oncogene, 1997. 14(12): p. 1419-26.

170. Fonseca, P.M., et al., Regulation and localization of CAS substrate domain tyrosine phosphorylation. Cell Signal, 2004. 16(5): p. 621-9.

171. Ballestrem, C., et al., Molecular mapping of tyrosine-phosphorylated proteins in focal adhesions using fluorescence resonance energy transfer. J Cell Sci, 2006. 119(Pt 5): p. 866-75.

172. Sawada, Y., et al., Force sensing by mechanical extension of the Src family kinase substrate p130Cas. Cell, 2006. 127(5): p. 1015-26.

173. Meenderink, L.M., et al., P130Cas Src-binding and substrate domains have distinct roles in sustaining focal adhesion disassembly and promoting cell migration. PLoS One, 2010. 5(10): p. e13412.

174. Cunningham-Edmondson, A.C. and S.K. Hanks, p130Cas substrate domain signaling promotes migration, invasion, and survival of estrogen receptornegative breast cancer cells. Breast Cancer (London), 2009. 2009(1): p. 39-52. 
175. Schlaepfer, D.D., M.A. Broome, and T. Hunter, Fibronectin-stimulated signaling from a focal adhesion kinase-c-Src complex: involvement of the Grb2, p130cas, and Nck adaptor proteins. Mol Cell Biol, 1997. 17(3): p. 1702-13.

176. Shin, N.Y., et al., Subsets of the major tyrosine phosphorylation sites in Crkassociated substrate (CAS) are sufficient to promote cell migration. J Biol Chem, 2004. 279(37): p. 38331-7.

177. Briknarova, K., et al., The serine-rich domain from Crk-associated substrate (p130cas) is a four-helix bundle. J Biol Chem, 2005. 280(23): p. 21908-14.

178. Garcia-Guzman, M., et al., Cell adhesion regulates the interaction between the docking protein p130(Cas) and the 14-3-3 proteins. J Biol Chem, 1999. 274(9): p. 5762-8.

179. Nakamoto, T., et al., Direct binding of C-terminal region of p130Cas to SH2 and SH3 domains of Src kinase. J Biol Chem, 1996. 271(15): p. 8959-65.

180. Nakamoto, T., et al., Requirements for localization of p130cas to focal adhesions. Mol Cell Biol, 1997. 17(7): p. 3884-97.

181. Donaldson, J.C., et al., Crk-associated substrate p130(Cas) interacts with nephrocystin and both proteins localize to cell-cell contacts of polarized epithelial cells. Exp Cell Res, 2000. 256(1): p. 168-78.

182. Li, E., et al., Association of p130CAS with phosphatidylinositol-3-OH kinase mediates adenovirus cell entry. J Biol Chem, 2000. 275(19): p. 14729-35.

183. Arold, S.T., M.K. Hoellerer, and M.E. Noble, The structural basis of localization and signaling by the focal adhesion targeting domain. Structure, 2002. 10(3): p. 319-27.

184. Riggins, R.B., L.A. Quilliam, and A.H. Bouton, Synergistic promotion of c-Src activation and cell migration by Cas and AND-34/BCAR3. J Biol Chem, 2003. 278(30): p. 28264-73.

185. Pratt, S.J., et al., The LIM protein Ajuba influences p130Cas localization and Racl activity during cell migration. J Cell Biol, 2005. 168(5): p. 813-24.

186. Abassi, Y.A., et al., p130Cas Couples the tyrosine kinase Bmx/Etk with regulation of the actin cytoskeleton and cell migration. J Biol Chem, 2003. 278(37): p. 35636-43.

187. Lu, Y., J. Brush, and T.A. Stewart, NSP1 defines a novel family of adaptor proteins linking integrin and tyrosine kinase receptors to the c-Jun $N$-terminal kinase/stress-activated protein kinase signaling pathway. J Biol Chem, 1999. 274(15): p. 10047-52.

188. Sakakibara, A. and S. Hattori, Chat, a Cas/HEF1-associated adaptor protein that integrates multiple signaling pathways. J Biol Chem, 2000. 275(9): p. 6404-10.

189. Near, R.I., et al., AND-34/BCAR3 differs from other NSP homologs in induction of anti-estrogen resistance, cyclin D1 promoter activation and altered breast cancer cell morphology. J Cell Physiol, 2007. 212(3): p. 655-65.

190. Cai, D., et al., AND-34/BCAR3, a GDP exchange factor whose overexpression confers antiestrogen resistance, activates Rac, PAK1, and the cyclin D1 promoter. Cancer Res, 2003. 63(20): p. 6802-8.

191. van Agthoven, T., et al., Identification of BCAR3 by a random search for genes involved in antiestrogen resistance of human breast cancer cells. EMBO J, 1998. 17(10): p. 2799-808. 
192. Sakakibara, A., et al., Novel function of Chat in controlling cell adhesion via CasCrk-C3G-pathway-mediated Rapl activation. J Cell Sci, 2002. 115(Pt 24): p. 4915-24.

193. Wang, L., et al., The SRC homology 2 domain protein Shep1 plays an important role in the penetration of olfactory sensory axons into the forebrain. J Neurosci, 2010. 30(39): p. 13201-10.

194. Casamassima, A. and E. Rozengurt, Tyrosine phosphorylation of p130(cas) by bombesin, lysophosphatidic acid, phorbol esters, and platelet-derived growth factor. Signaling pathways and formation of a p130(cas)-Crk complex. J Biol Chem, 1997. 272(14): p. 9363-70.

195. Casamassima, A. and E. Rozengurt, Insulin-like growth factor I stimulates tyrosine phosphorylation of p130(Cas), focal adhesion kinase, and paxillin. Role of phosphatidylinositol 3'-kinase and formation of a p130(Cas).Crk complex. J Biol Chem, 1998. 273(40): p. 26149-56.

196. Lee, T.N., et al., Glucose stimulates the association of Crk with p130Cas in pancreatic beta cells. Pancreas, 2004. 29(4): p. e100-5.

197. Petruzzelli, L., M. Takami, and R. Herrera, Adhesion through the interaction of lymphocyte function-associated antigen-1 with intracellular adhesion molecule-1 induces tyrosine phosphorylation of p130cas and its association with c-CrkII. J Biol Chem, 1996. 271(13): p. 7796-801.

198. Tikhmyanova, N., et al., Dcas supports cell polarization and cell-cell adhesion complexes in development. PLoS One, 2010. 5(8): p. e12369.

199. Kovacic-Milivojevic, B., et al., Focal adhesion kinase and p130Cas mediate both sarcomeric organization and activation of genes associated with cardiac myocyte hypertrophy. Mol Biol Cell, 2001. 12(8): p. 2290-307.

200. Kovacic-Milivojevic, B., et al., Requirements for the localization of p130 Cas to Z-lines in cardiac myocytes. Cell Mol Biol Lett, 2002. 7(2): p. 323-9.

201. Wozniak, M.A., et al., The emerin-binding transcription factor Lmo 7 is regulated by association with p130Cas at focal adhesions. PeerJ, 2013. 1: p. e134.

202. Kawauchi, K., et al., p130Cas-dependent actin remodelling regulates myogenic differentiation. Biochem J, 2012. 445(3): p. 323-32.

203. Jeong da, E., et al., The 31-kDa caspase-generated cleavage product of p130Cas antagonizes the action of MyoD during myogenesis. Biochem Biophys Res Commun, 2014. 444(4): p. 509-13.

204. Huang, J., R. Sakai, and T. Furuichi, The docking protein Cas links tyrosine phosphorylation signaling to elongation of cerebellar granule cell axons. Mol Biol Cell, 2006. 17(7): p. 3187-96.

205. Huang, Z., et al., Crk-associated substrate (Cas) signaling protein functions with integrins to specify axon guidance during development. Development, 2007. 134(12): p. 2337-47.

206. Riccomagno, M.M., et al., Cas adaptor proteins organize the retinal ganglion cell layer downstream of integrin signaling. Neuron, 2014. 81(4): p. 779-86.

207. Gonsior, C., et al., Oligodendroglial p130Cas is a target of Fyn kinase involved in process formation, cell migration and survival. PLoS One, 2014. 9(2): p. e89423.

208. Wei, L., et al., Anchorage-independent phosphorylation of p130(Cas) protects lung adenocarcinoma cells from anoikis. J Cell Biochem, 2002. 87(4): p. 439-49. 
209. Giancotti, F.G. and G. Tarone, Positional control of cell fate through joint integrin/receptor protein kinase signaling. Annu Rev Cell Dev Biol, 2003. 19: p. 173-206.

210. Kim, W., et al., The 31-kDa caspase-generated cleavage product of p130cas functions as a transcriptional repressor of E2A in apoptotic cells. J Biol Chem, 2004. 279(9): p. 8333-42.

211. Dolfi, F., et al., The adaptor protein Crk connects multiple cellular stimuli to the JNK signaling pathway. Proc Natl Acad Sci U S A, 1998. 95(26): p. 15394-9.

212. Oktay, M., et al., Integrin-mediated activation of focal adhesion kinase is required for signaling to Jun NH2-terminal kinase and progression through the G1 phase of the cell cycle. J Cell Biol, 1999. 145(7): p. 1461-9.

213. Janssen, H. and P. Marynen, Interaction partners for human ZNF384/CIZ/NMP4-zyxin as a mediator for p130CAS signaling? Exp Cell Res, 2006. 312(7): p. 1194-204.

214. Yi, J., et al., Members of the Zyxin family of LIM proteins interact with members of the p130Cas family of signal transducers. J Biol Chem, 2002. 277(11): p. 9580-9.

215. Dorssers, L.C., et al., The prognostic value of BCAR1 in patients with primary breast cancer. Clin Cancer Res, 2004. 10(18 Pt 1): p. 6194-202.

216. Konstantinovsky, S., et al., Breast carcinoma cells in primary tumors and effusions have different gene array profiles. J Oncol, 2010. 2010: p. 969084.

217. Cabodi, S., et al., p130Cas is an essential transducer element in ErbB2 transformation. FASEB J, 2010. 24(10): p. 3796-808.

218. Tornillo, G., et al., p130Cas promotes invasiveness of three-dimensional ErbB2transformed mammary acinar structures by enhanced activation of $m T O R / p 70 S 6 K$ and Racl. Eur J Cell Biol, 2011. 90(2-3): p. 237-48.

219. Salgia, R., et al., p130CAS forms a signaling complex with the adapter protein $C R K L$ in hematopoietic cells transformed by the BCR/ABL oncogene. J Biol Chem, 1996. 271(41): p. 25198-203.

220. Cho, Y.J., et al., Interaction of $B c r / A b l$ with $C 3 G$, an exchange factor for the small GTPase Rapl, through the adapter protein Crkl. Biochem Biophys Res Commun, 2005. 333(4): p. 1276-83.

221. Tachibana, K., et al., Direct association of pp125FAK with paxillin, the focal adhesion-targeting mechanism of pp 125FAK. J Exp Med, 1995. 182(4): p. 108999.

222. Nolan, K., J. Lacoste, and J.T. Parsons, Regulated expression of focal adhesion kinase-related nonkinase, the autonomously expressed C-terminal domain of focal adhesion kinase. Mol Cell Biol, 1999. 19(9): p. 6120-9.

223. Richardson, A. and T. Parsons, A mechanism for regulation of the adhesionassociated proteintyrosine kinase pp125FAK. Nature, 1996. 380(6574): p. 53840.

224. Cary, L.A. and J.L. Guan, Focal adhesion kinase in integrin-mediated signaling. Front Biosci, 1999. 4: p. D102-13.

225. Schlaepfer, D.D., C.R. Hauck, and D.J. Sieg, Signaling through focal adhesion kinase. Prog Biophys Mol Biol, 1999. 71(3-4): p. 435-78. 
226. Gilmore, A.P. and L.H. Romer, Inhibition of focal adhesion kinase (FAK) signaling in focal adhesions decreases cell motility and proliferation. Mol Biol Cell, 1996. 7(8): p. 1209-24.

227. Shen, Y., et al., Direct association of protein-tyrosine phosphatase PTP-PEST with paxillin. J Biol Chem, 1998. 273(11): p. 6474-81.

228. Thomas, J.W., et al., The role of focal adhesion kinase binding in the regulation of tyrosine phosphorylation of paxillin. J Biol Chem, 1999. 274(51): p. 36684-92.

229. Worrall, J.A., et al., Transient protein interactions studied by NMR spectroscopy: the case of cytochrome $C$ and adrenodoxin. Biochemistry, 2003. 42(23): p. 706876.

230. Mace, P.D., et al., NSP-Cas protein structures reveal a promiscuous interaction module in cell signaling. Nat Struct Mol Biol, 2011. 18(12): p. 1381-7.

231. Nikolopoulos, S.N. and C.E. Turner, Integrin-linked kinase (ILK) binding to paxillin LDI motif regulates ILK localization to focal adhesions. J Biol Chem, 2001. 276(26): p. 23499-505.

232. Lorenz, S., et al., Structural analysis of the interactions between paxillin LD motifs and alpha-parvin. Structure, 2008. 16(10): p. 1521-31.

233. Nikolopoulos, S.N. and C.E. Turner, Actopaxin, a new focal adhesion protein that binds paxillin LD motifs and actin and regulates cell adhesion. J Cell Biol, 2000. 151(7): p. 1435-48.

234. Vanarotti, M.S., et al., Structural and mechanistic insights into the interaction between Pyk2 and paxillin LD motifs. J Mol Biol, 2014. 426(24): p. 3985-4001.

235. F. Ruan, Y.C., and P. B. Hopkins, Metal ion enhanced helicity in synthetic peptides containing unnatural, metal-ligating residues. Journal of the American Chemical Society, 1990. 112, no. 25, pp. 9403-9404.

236. Stewart, M.L., et al., The MCL-1 BH3 helix is an exclusive MCL-1 inhibitor and apoptosis sensitizer. Nat Chem Biol, 2010. 6(8): p. 595-601. 


\section{VITA}

Chi Zhang was born in Shenyang, Liaoning, China in 1982. In July of 2004, he completed a Bachelor Degree of Biology in Shenzhen University, and then joined Microchip Inc. from 2005 to 2007. He enrolled in the IPBS program at the University of Tennessee Health Center in 2007. He joined the Zheng laboratory at the St. Jude Children's Research Hospital in 2008. He is expected to graduate in May 2015. 